\title{
Synthesis of cationic rhenium(VII) oxo-imido complexes and their tunability towards oxygen atom transfer
}

Elon A. Ison, Jeanette E. Cessarich, Nicholos E. Travia, Phillip E. Fanwick, Mahdi M.

Abu-Omar ${ }^{*}$

Brown Laboratory, Department of Chemistry; Purdue University,

Supporting Information

Kinetic profile for the reaction of $\mathbf{5 a}$ with $\mathrm{PPh}_{3}$

Figure S1

Hammett analysis for the reaction of $\mathrm{PAr}_{3}$ with $\mathbf{5 a}$

Figure S2

Kinetic profile for the reaction of $5 \mathbf{a}$ with $\mathrm{P}\left(p-\mathrm{CF}_{3} \mathrm{Ph}\right)_{3}$

Figure S3

Kinetic profile for the reaction of $\mathbf{5 a}$ with $\mathrm{P}(\mathrm{p}-\mathrm{OMePh})_{3}$

Figure S4

MeSPh dependence for the reaction of $\left[\operatorname{Re}(\mathrm{O})\left(\mathrm{NTos} \mathrm{saldach}^{+}\right]\right.$

with PhSMe

Figure S5

HSPh dependence for the reaction of $\left[\operatorname{Re}(\mathrm{O})(\mathrm{NAr}) \mathrm{saldach}^{+}\right]$

( $\mathrm{Ar}=p-\mathrm{CF}_{3}$ phenyl] with $\mathrm{HSPh}$

Figure S6

$\mathrm{PPh}_{3}$ dependence for the reaction of $\left[\mathrm{Re}(\mathrm{O})(\mathrm{NAr}) \mathrm{saldach}^{+}\right]$

$\left(\mathrm{Ar}=p-\mathrm{CF}_{3}\right.$ phenyl $]$ with $\mathrm{PPh}_{3}$

Figure S7

$\mathrm{PPh}_{3}$ dependence for the reaction of $\left[\mathrm{Re}(\mathrm{O})(\mathrm{NMes}) \mathrm{saldach}^{+}\right]$

with $\mathrm{PPh}_{3}$

Figure S8

$\mathrm{P}\left(p-\mathrm{CF}_{3} \mathrm{Ph}\right)_{3}$ dependence for the reaction of $\left[\mathrm{Re}(\mathrm{O})(\mathrm{NMes}) \mathrm{saldach}^{+}\right]$ 
with $\mathrm{P}\left(p-\mathrm{CF}_{3} \mathrm{Ph}\right)_{3}$

$\mathrm{P}(p-\mathrm{OMePh})_{3}$ dependence for the reaction of $\left[\mathrm{Re}(\mathrm{O})(\mathrm{NMes}) \mathrm{saldach}^{+}\right]$ with $\mathrm{P}(p-\mathrm{OMePh})_{3}$

Crystal structure data for $\mathbf{5 a}$

Crystal structure data for $\mathbf{7 a}$
Figure S9

Figure S10

Tables S1-S4

Tables S5-S8 


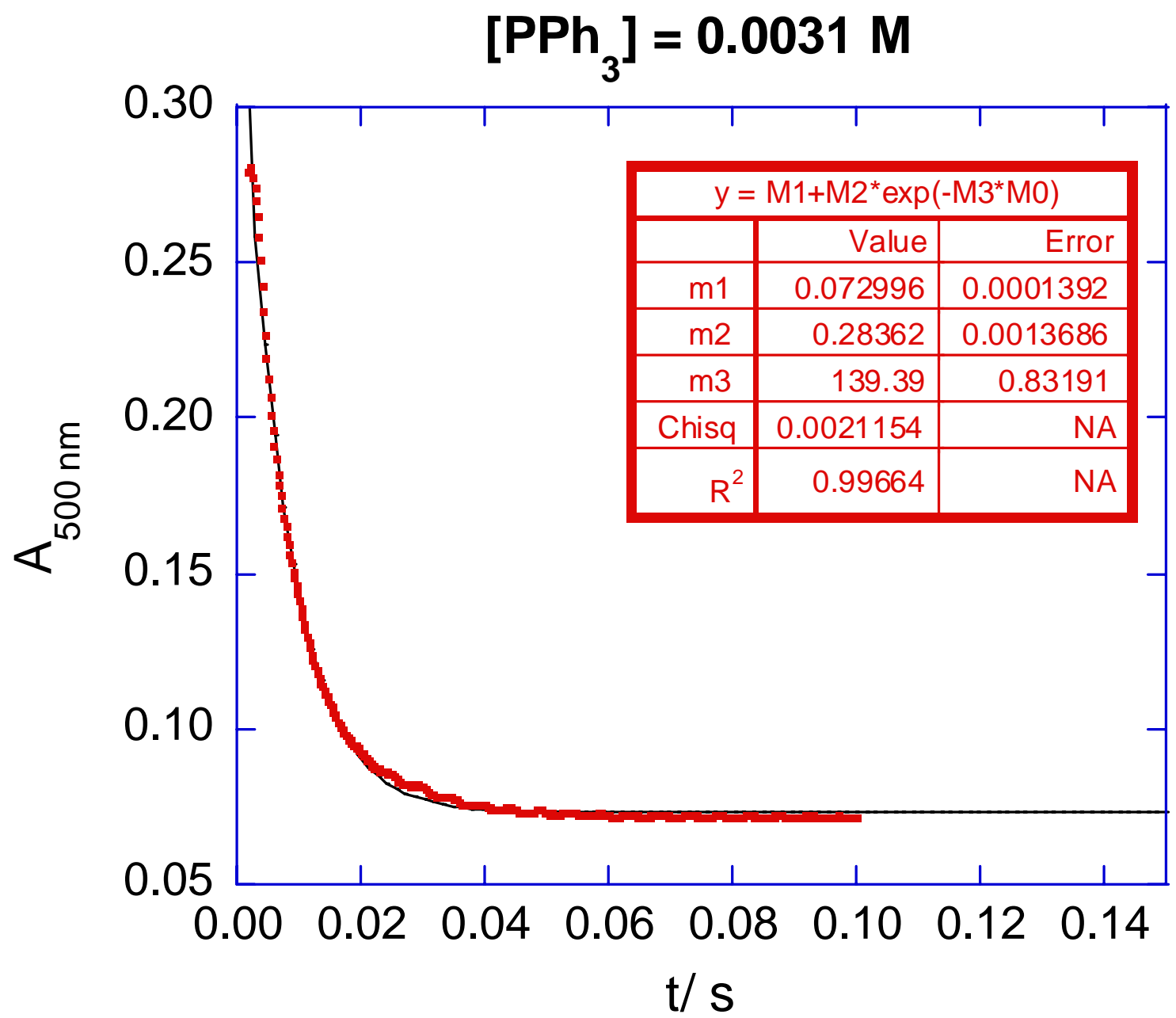

Figure S1. A representative kinetic profile for the disappearance of [(saldach) $\left.\operatorname{Re}(\mathrm{O})(\mathrm{NMes})^{+}\right], \mathbf{5 a}$, from its reaction with $\mathrm{PPh}_{3}$. Conditions [5a] $=0.1 \mathrm{mM}$ and $\left[\mathrm{PPh}_{3}\right]=0.0031 \mathrm{M}$ in $\mathrm{CH}_{3} \mathrm{CN}$ at $298 \mathrm{~K}$ at $500 \mathrm{~nm}$. Data was fit to a pseudo first order equation $\mathrm{Abs}_{\mathrm{t}}=\mathrm{Abs}_{\infty}+\left(\mathrm{Abs}_{0}-\mathrm{Abs}_{\infty}\right) \exp \left(-k_{\psi} \mathrm{t}\right)$. 


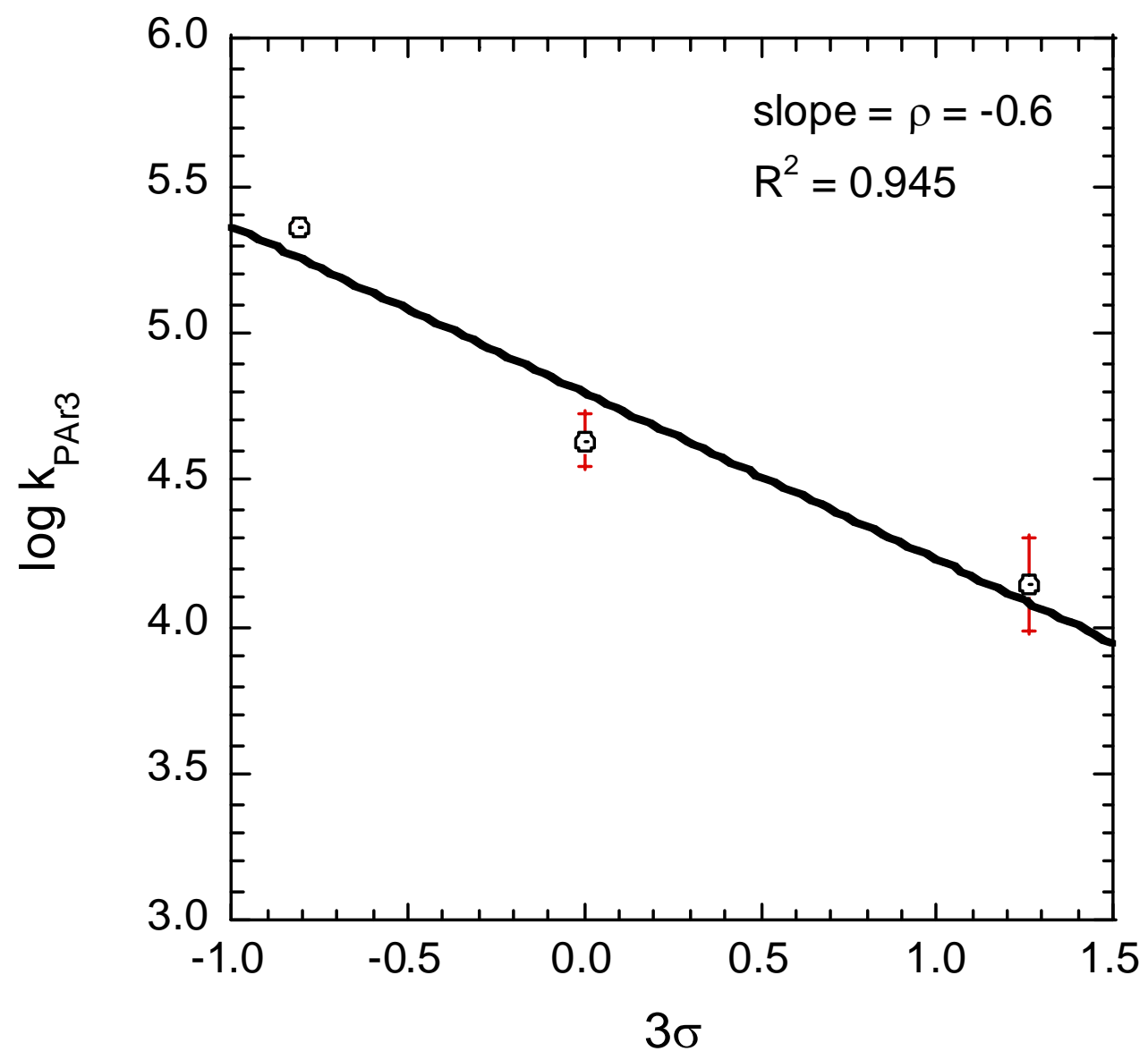

Figures S2. Hammett analysis for the reaction of $5 \mathbf{a}$ with $\mathrm{Par}_{3}(\mathrm{Ar}=p-\mathrm{OMePh}, \mathrm{Ph}$, and $p-$ $\left.\mathrm{CF}_{3} \mathrm{Ph}\right)$. 


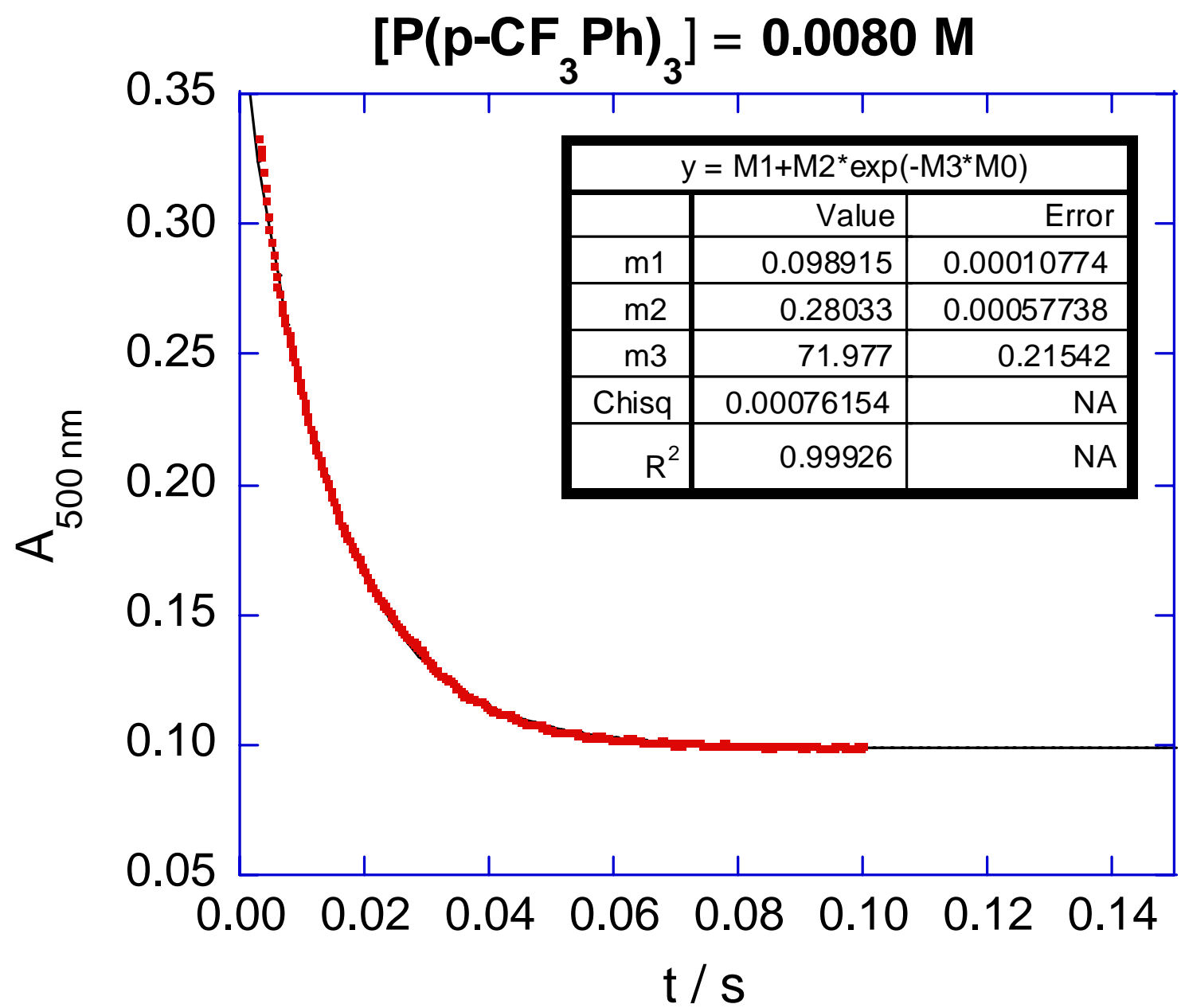

Figure S3. A representative kinetic profile for the disappearance of $[$ (saldach $\left.) \operatorname{Re}(\mathrm{O})(\mathrm{NMes})^{+}\right], \mathbf{5 a}$, from its reaction with $\mathrm{P}\left(p-\mathrm{CF}_{3} \mathrm{Ph}\right)_{3}$. Conditions $[\mathbf{5 a}]=0.1$ $\mathrm{mM}$ and $\left[\mathrm{P}\left(p-\mathrm{CF}_{3} \mathrm{Ph}\right)_{3}\right]=0.0080 \mathrm{M}$ in $\mathrm{CH}_{3} \mathrm{CN}$ at $298 \mathrm{~K}$ at $500 \mathrm{~nm}$. Data was fit to a pseudo first order equation $\mathrm{Abs}_{\mathrm{t}}=\mathrm{Abs}_{\infty}+\left(\mathrm{Abs}_{0}-\mathrm{Abs}_{\infty}\right) \exp \left(-k_{\psi} \mathrm{t}\right)$. 


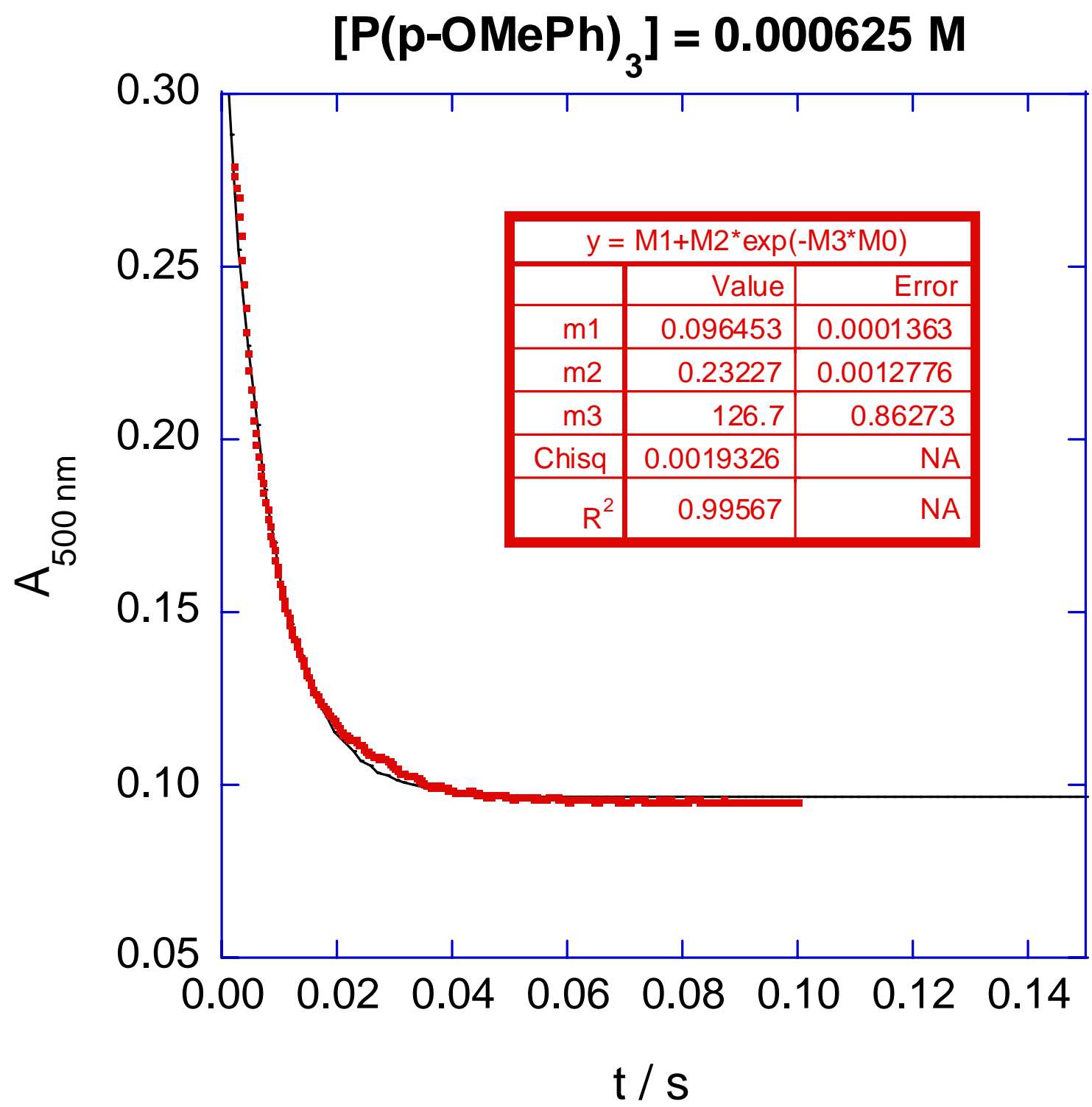

Figure S4. A representative kinetic profile for the disappearance of $[$ (saldach $\left.) \operatorname{Re}(\mathrm{O})(\mathrm{NMes})^{+}\right], 5 a$, from its reaction with $\mathrm{P}(p-\mathrm{OMePh})_{3}$. Conditions $[5 \mathbf{a}]=$ $0.10 \mathrm{mM}$ and $\left[\mathrm{P}(p-\mathrm{OMePh})_{3}\right]=0.000625 \mathrm{M}$ in $\mathrm{CH}_{3} \mathrm{CN}$ at $298 \mathrm{~K}$ at $500 \mathrm{~nm}$. Data was fit to a pseudo first order equation $\mathrm{Abs}_{\mathrm{t}}=\mathrm{Abs}_{\infty}+\left(\mathrm{Abs}_{0}-\mathrm{Abs}_{\infty}\right) \exp \left(-k_{\psi} \mathrm{t}\right)$. 


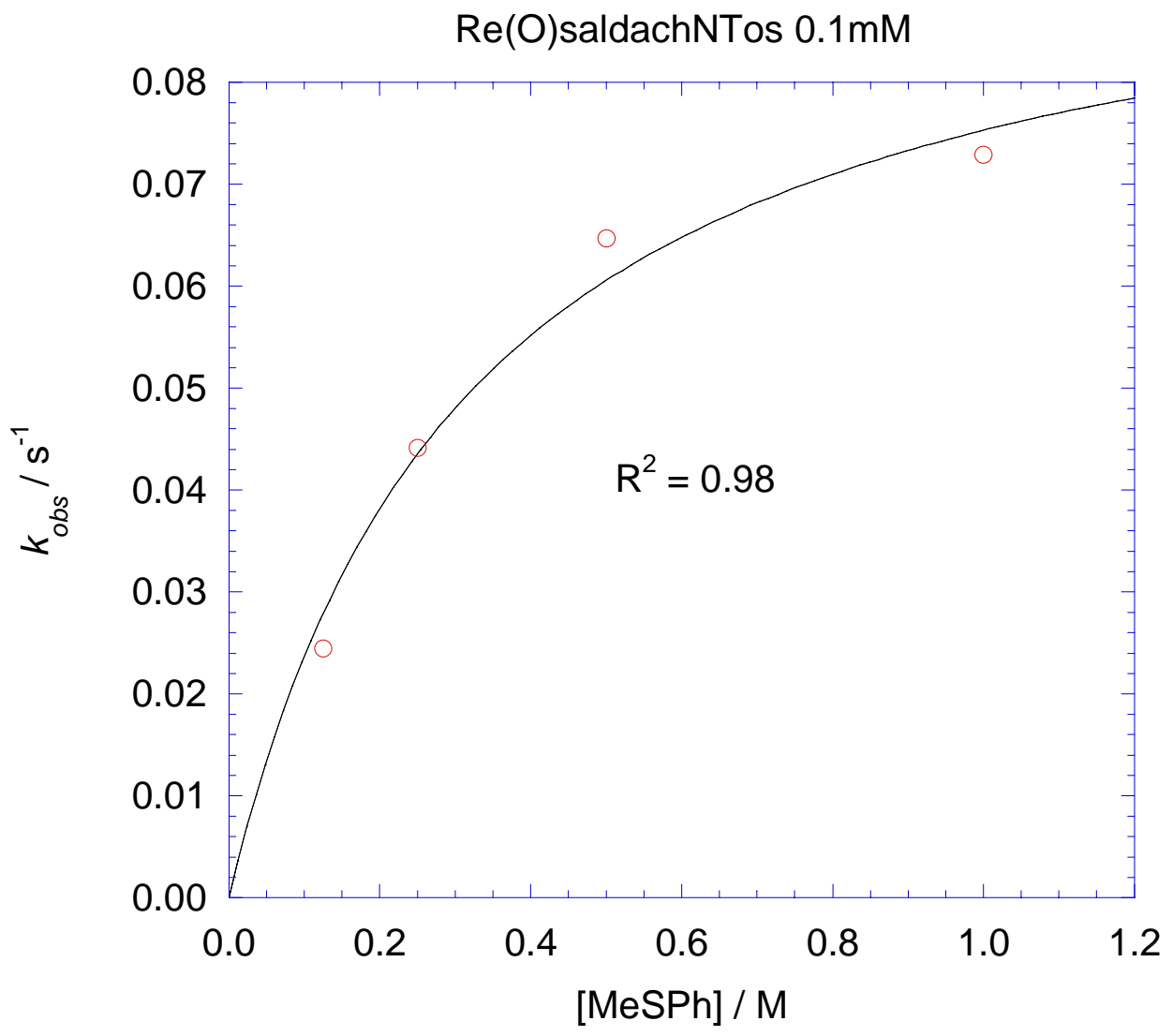

Figure S5. MeSPh dependence for the reaction of $\left[(\right.$ saldach $\left.) \operatorname{Re}(\mathrm{O})(\mathrm{NTos})^{+}\right]$with $\mathrm{MeSPh}$. Conditions [(saldach) $\left.\mathrm{Re}(\mathrm{O})(\mathrm{NTos})^{+}\right]=0.1 \mathrm{mM}$ in $\mathrm{CH}_{3} \mathrm{CN}$ at $298 \mathrm{~K}$ at $500 \mathrm{~nm}$. 


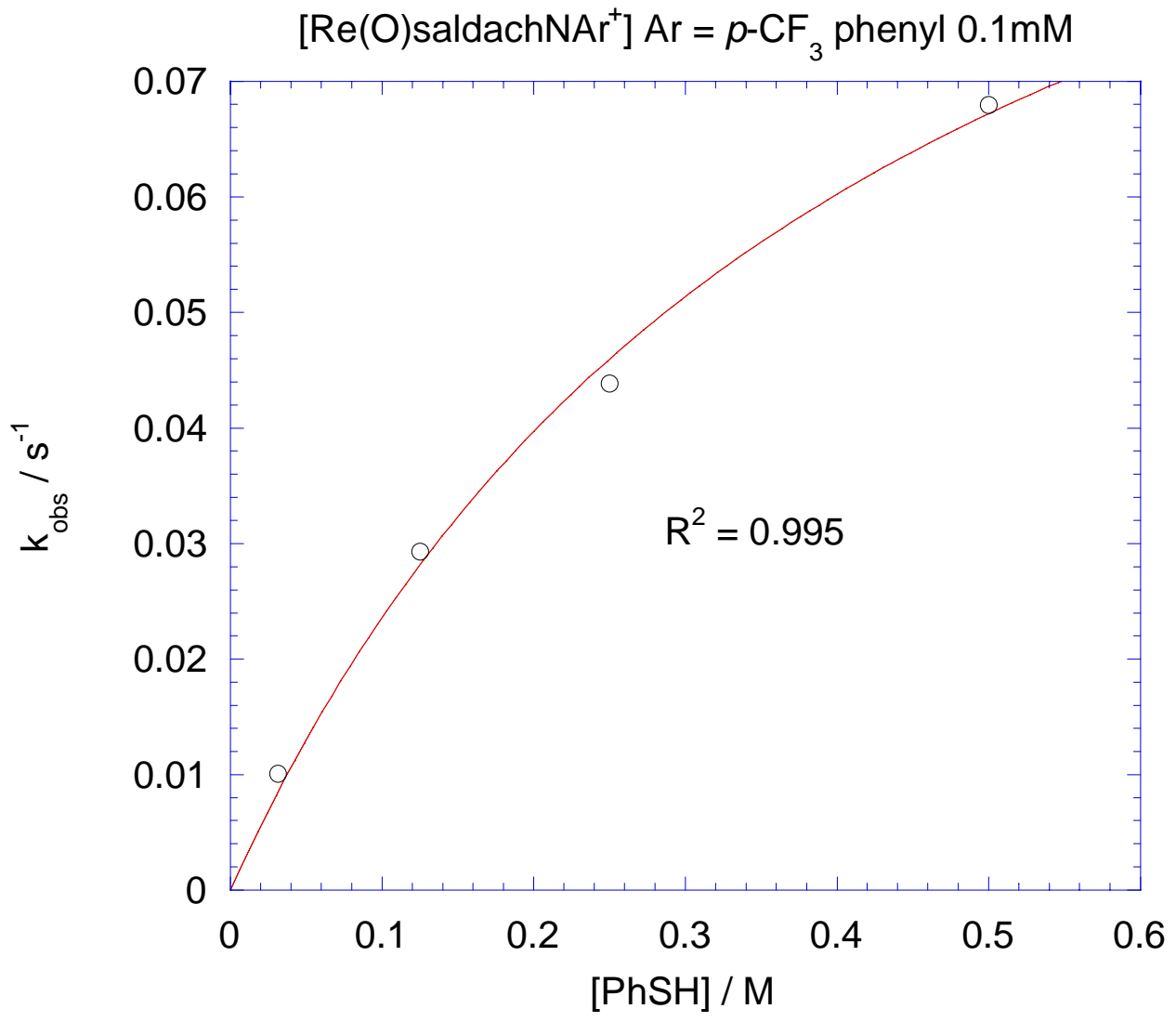

Figure S6. HSPh dependence for the reaction of $\left[(\right.$ saldach $\left.) \operatorname{Re}(\mathrm{O})(\mathrm{NAr})^{+}\right]\left(\mathrm{Ar}=p-\mathrm{CF}_{3}\right.$ phenyl] with $\mathrm{HSPh}$. Conditions [(saldach) $\left.\mathrm{Re}(\mathrm{O})(\mathrm{NAr})^{+}\right]=0.1 \mathrm{mM}$ in $\mathrm{CH}_{3} \mathrm{CN}$ at $298 \mathrm{~K}$ at $500 \mathrm{~nm}$. 


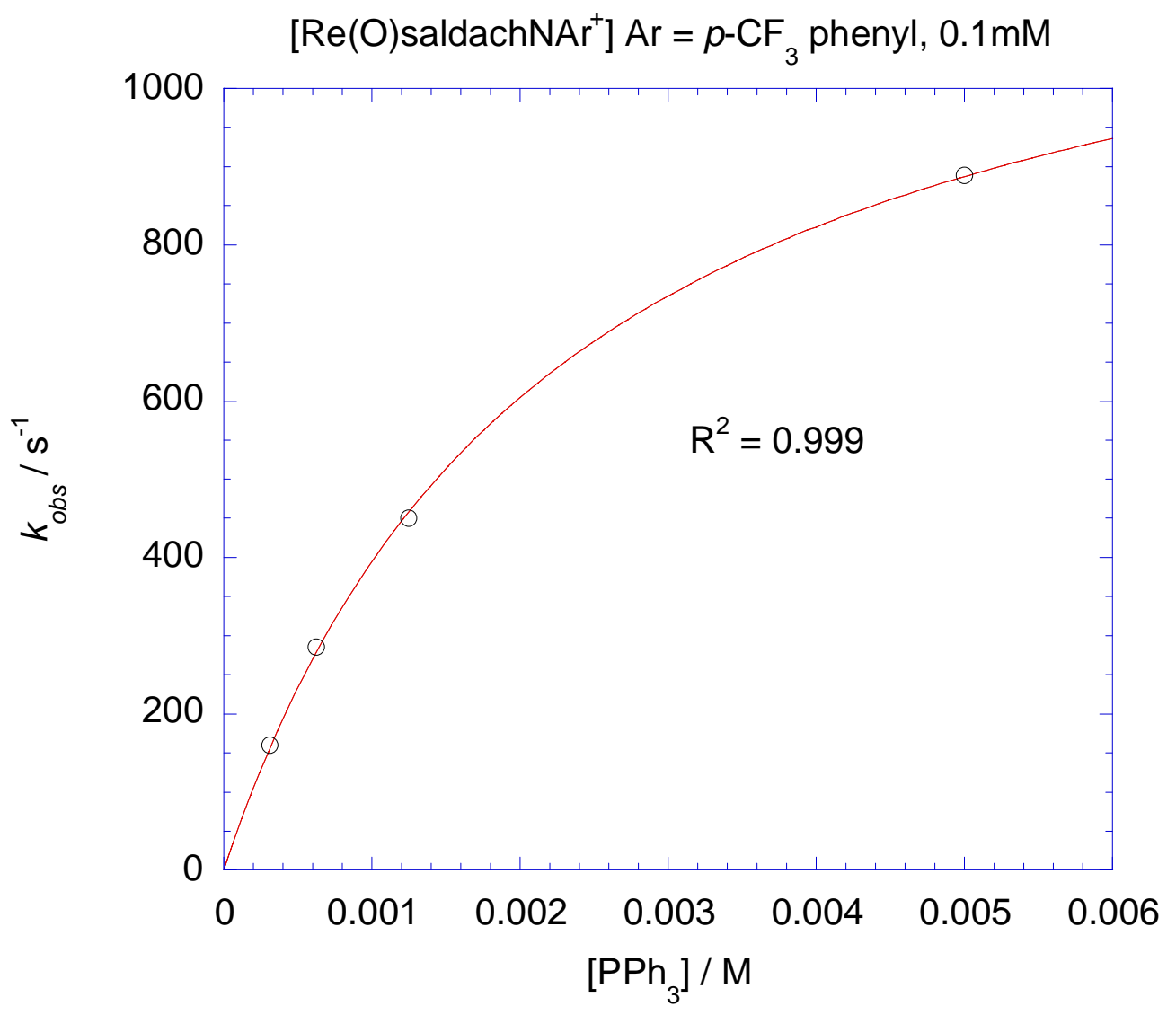

Figure S7. $\mathrm{PPh}_{3}$ dependence for the reaction of $\left[(\right.$ saldach $\left.) \operatorname{Re}(\mathrm{O})(\mathrm{NAr})^{+}\right]\left(\mathrm{Ar}=p-\mathrm{CF}_{3}\right.$ phenyl] with $\mathrm{PPh}_{3}$. Conditions [(saldach) $\left.\mathrm{Re}(\mathrm{O})(\mathrm{NAr})^{+}\right]=0.1 \mathrm{mM}$ in $\mathrm{CH}_{3} \mathrm{CN}$ at $298 \mathrm{~K}$ at $500 \mathrm{~nm}$. 


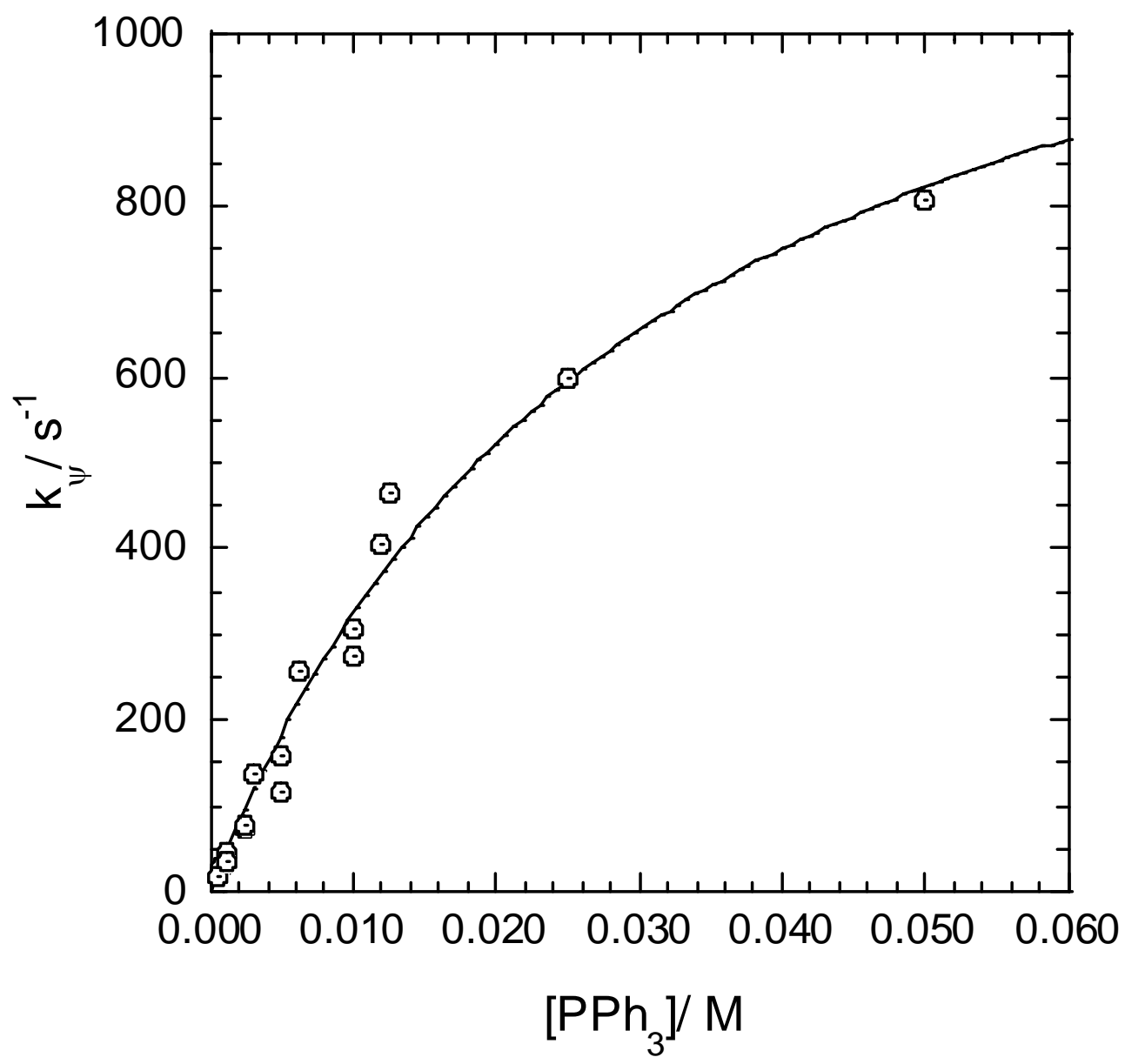

Figure S8. $\mathrm{PPh}_{3}$ dependence for the reaction of $\left[(\right.$ saldach $\left.) \mathrm{Re}(\mathrm{O})(\mathrm{NMes})^{+}\right]$with $\mathrm{PPh}_{3}$. Conditions [(saldach) $\left.\mathrm{Re}(\mathrm{O})(\mathrm{NMes})^{+}\right]=0.1 \mathrm{mM}$ in $\mathrm{CH}_{3} \mathrm{CN}$ at $298 \mathrm{~K}$. 


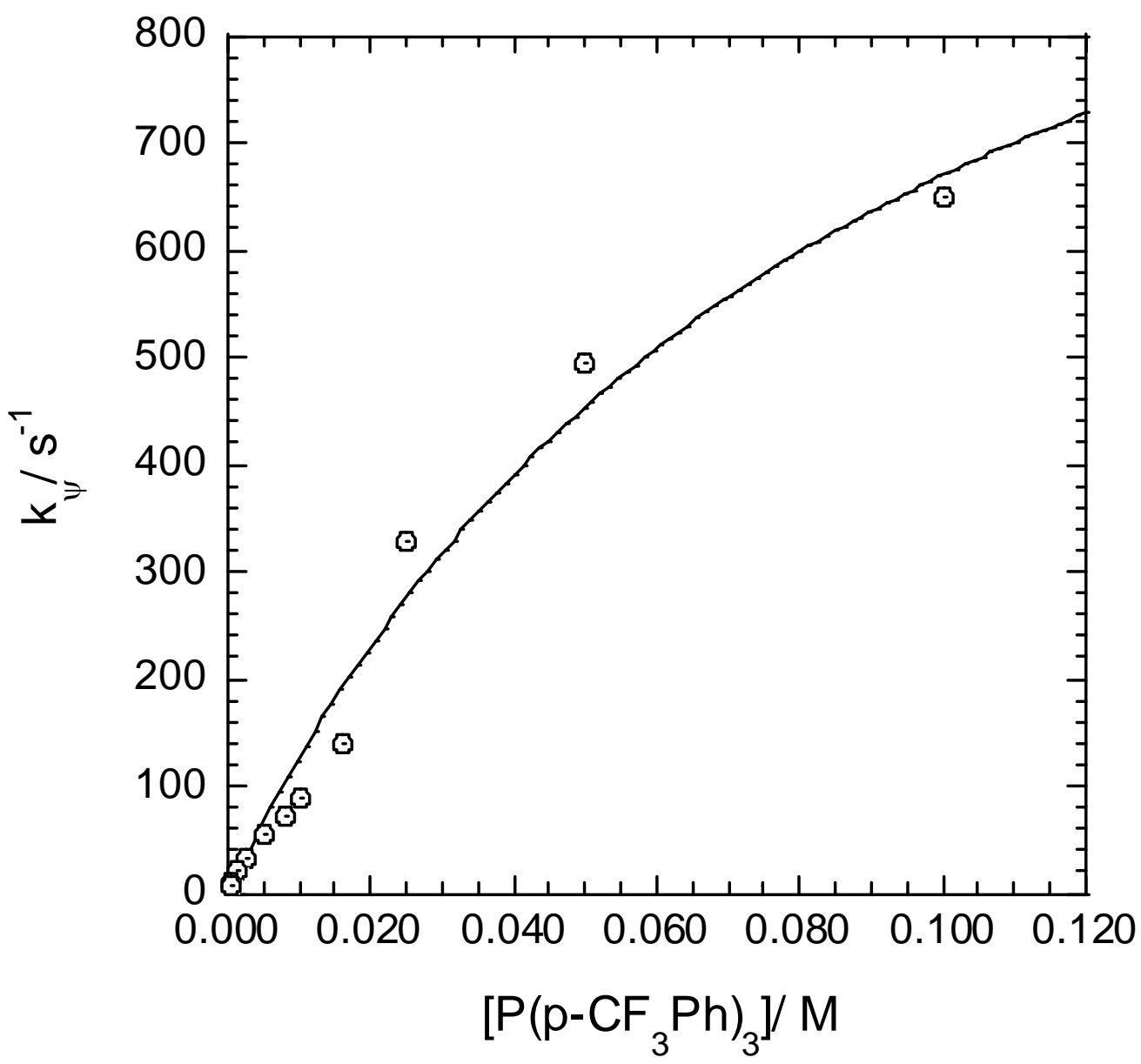

Figure S9. $\mathrm{P}\left(p-\mathrm{CF}_{3} \mathrm{Ph}\right)_{3}$ dependence for the reaction of $\left[(\right.$ saldach $\left.) \mathrm{Re}(\mathrm{O})(\mathrm{NMes})^{+}\right]$with

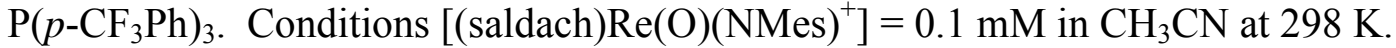




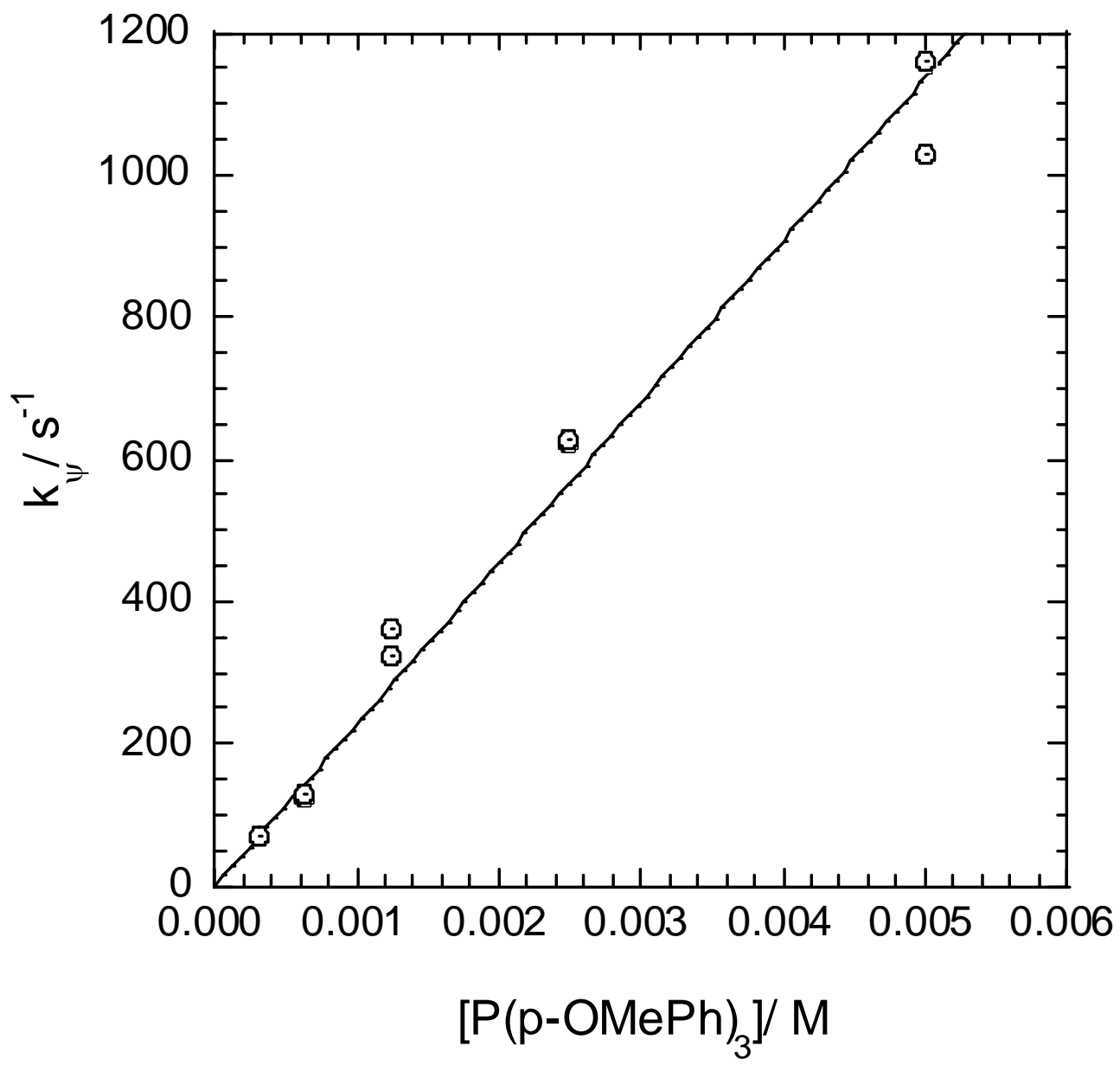

Figure $\mathrm{S} 10 . \mathrm{P}(p-\mathrm{OMePh})_{3}$ dependence for the reaction of $\left[(\right.$ saldach $\left.) \mathrm{Re}(\mathrm{O})(\mathrm{NMes})^{+}\right]$with $\mathrm{P}(p-\mathrm{OMePh})_{3}$. Conditions $\left[(\right.$ saldach $\left.) \mathrm{Re}(\mathrm{O})(\mathrm{NMes})^{+}\right]=0.1 \mathrm{mM}$ in $\mathrm{CH}_{3} \mathrm{CN}$ at $298 \mathrm{~K}$. 


\section{X-ray Data}

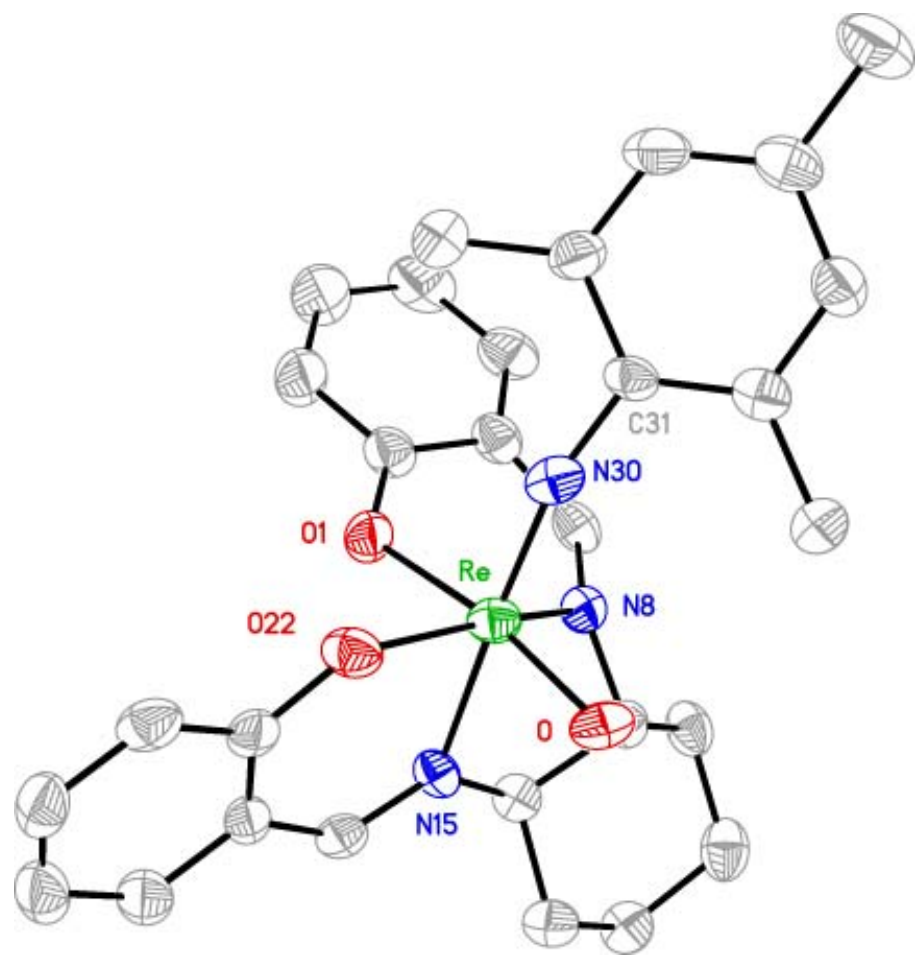

Table S1. Bond Angles in Degrees for $\left[\operatorname{Re}(\mathrm{O})\left(\mathrm{NMes} \mathrm{saldach}^{+}\right], \mathbf{5 a}\right.$

\begin{tabular}{|c|c|c|c|c|c|c|}
\hline Angle $^{\underline{\text { Atom } 1}}$ & $\underline{\text { Atom } 2}$ & Atom $\underline{3}$ & $\underline{\text { Angle }}$ & $\underline{\text { Atom } 1}$ & $\underline{\text { Atom }} \underline{2}$ & $\underline{\text { Atom } 3}$ \\
\hline $\begin{array}{l}\mathrm{O} \\
108.3(4)\end{array}$ & $\operatorname{Re}$ & N30 & $100.89(17)$ & N8 & C9 & H9 \\
\hline $\begin{array}{l}\mathrm{O} \\
108.3(4)\end{array}$ & $\operatorname{Re}$ & $\mathrm{O} 22$ & $102.02(15)$ & $\mathrm{C} 10$ & C9 & H9 \\
\hline $\begin{array}{l}\text { N30 } \\
108.3(4)\end{array}$ & $\operatorname{Re}$ & $\mathrm{O} 22$ & $103.29(15)$ & C14 & C9 & H9 \\
\hline $\begin{array}{l}\mathrm{O} \\
107.1(4)\end{array}$ & $\operatorname{Re}$ & O1 & $163.38(14)$ & C9 & $\mathrm{C} 10$ & $\mathrm{C} 11$ \\
\hline $\begin{array}{l}\text { N30 } \\
110.3(4)\end{array}$ & $\mathrm{Re}$ & $\mathrm{O} 1$ & $93.47(16)$ & C9 & $\mathrm{C} 10$ & H10A \\
\hline $\begin{array}{l}\mathrm{O} 22 \\
110.3(4)\end{array}$ & $\operatorname{Re}$ & $\mathrm{O} 1$ & $82.52(14)$ & $\mathrm{C} 11$ & $\mathrm{C} 10$ & H10A \\
\hline $\begin{array}{l}\mathrm{O} \\
110.3(4)\end{array}$ & $\mathrm{Re}$ & N8 & $90.00(15)$ & C9 & $\mathrm{C} 10$ & H10B \\
\hline $\begin{array}{l}\text { N30 } \\
110.3(4)\end{array}$ & $\operatorname{Re}$ & N8 & $94.01(16)$ & C11 & $\mathrm{C} 10$ & H10B \\
\hline $\begin{array}{l}\mathrm{O} 22 \\
108.5(4)\end{array}$ & $\operatorname{Re}$ & N8 & $156.47(15)$ & H10A & $\mathrm{C} 10$ & H10B \\
\hline $\begin{array}{l}\text { O1 } \\
111.9(4)\end{array}$ & $\operatorname{Re}$ & $\mathrm{N} 8$ & $80.62(14)$ & $\mathrm{C} 12$ & $\mathrm{C} 11$ & $\mathrm{C} 10$ \\
\hline $\begin{array}{l}\mathrm{O} \\
109.2(4)\end{array}$ & $\operatorname{Re}$ & N15 & $82.33(15)$ & $\mathrm{C} 12$ & $\mathrm{C} 11$ & H11A \\
\hline N30 & $\operatorname{Re}$ & N15 & $167.98(15)$ & $\mathrm{C} 10$ & C11 & H11A \\
\hline
\end{tabular}




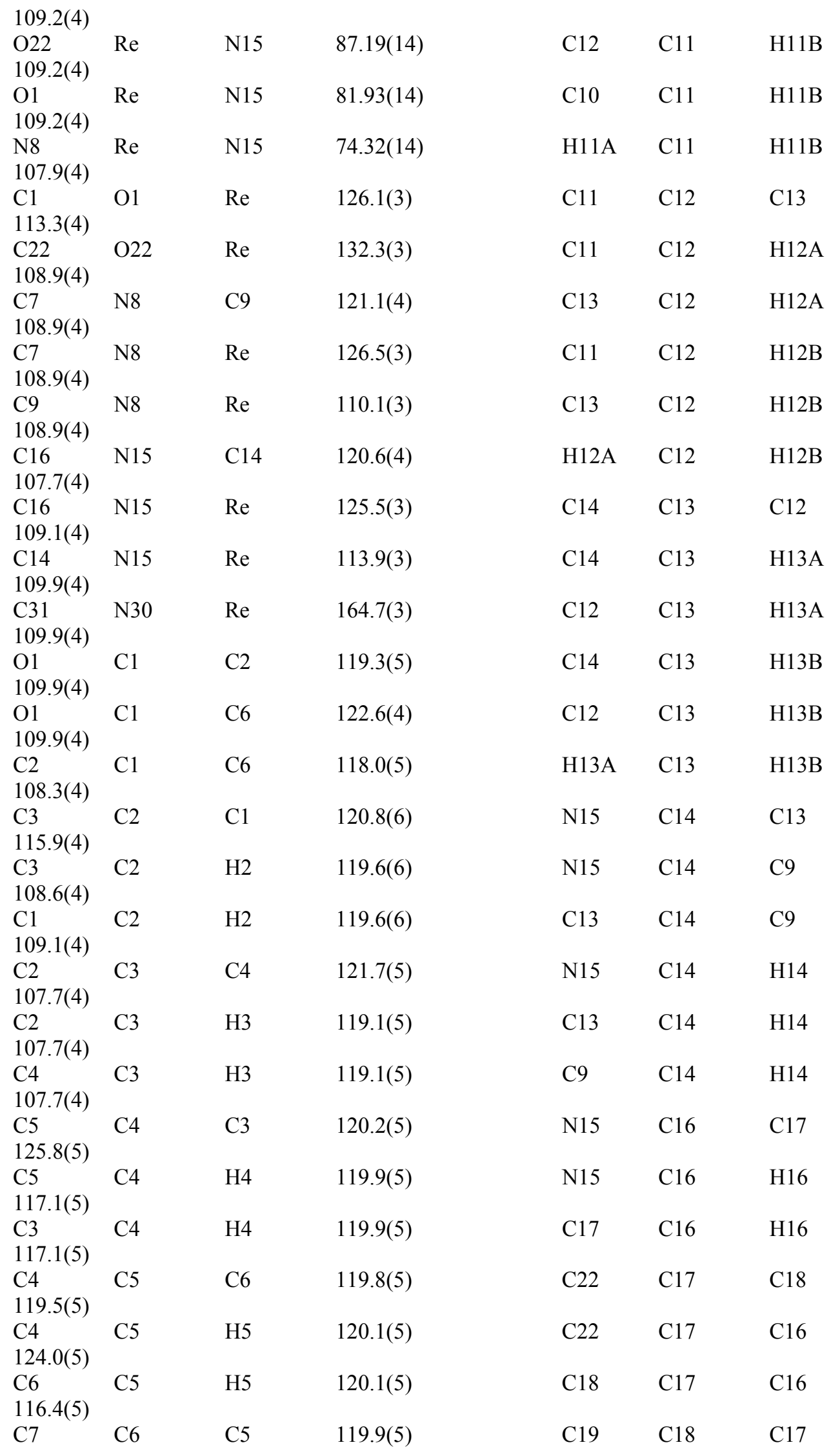




\begin{tabular}{|c|c|c|c|c|c|c|}
\hline $\begin{array}{l}120.7(5) \\
\text { C7 } \\
1196(5)\end{array}$ & C6 & $\mathrm{C} 1$ & $118.9(4)$ & C19 & $\mathrm{C} 18$ & H18 \\
\hline $\begin{array}{l}\text { C5 } \\
119.6(5)\end{array}$ & C6 & $\mathrm{C} 1$ & $119.4(5)$ & $\mathrm{C} 17$ & $\mathrm{C} 18$ & H18 \\
\hline $\begin{array}{l}\text { N8 } \\
119.4(5)\end{array}$ & $\mathrm{C} 7$ & C6 & $125.6(5)$ & $\mathrm{C} 18$ & C19 & $\mathrm{C} 20$ \\
\hline $\begin{array}{l}\text { N8 } \\
120.3(5)\end{array}$ & $\mathrm{C} 7$ & $\mathrm{H} 7$ & $117.2(5)$ & $\mathrm{C} 18$ & C19 & H19 \\
\hline $\begin{array}{l}\text { C6 } \\
120.3(5)\end{array}$ & $\mathrm{C} 7$ & H7 & $117.2(5)$ & $\mathrm{C} 20$ & C19 & H19 \\
\hline $\begin{array}{l}\text { N8 } \\
120.6(5)\end{array}$ & C9 & $\mathrm{C} 10$ & $117.8(4)$ & $\mathrm{C} 21$ & $\mathrm{C} 20$ & $\mathrm{C} 19$ \\
\hline $\begin{array}{l}\text { N8 } \\
119.7(5)\end{array}$ & C9 & $\mathrm{C} 14$ & 104.1(3) & $\mathrm{C} 21$ & $\mathrm{C} 20$ & $\mathrm{H} 20$ \\
\hline $\begin{array}{l}\mathrm{C} 10 \\
119.7(5)\end{array}$ & C9 & $\mathrm{C} 14$ & $109.9(4)$ & $\mathrm{C} 19$ & $\mathrm{C} 20$ & $\mathrm{H} 20$ \\
\hline $\begin{array}{l}\mathrm{C} 20 \\
120.6(5)\end{array}$ & $\mathrm{C} 21$ & $\mathrm{C} 22$ & $120.4(5)$ & F413 & $\mathrm{C} 413$ & C412 \\
\hline $\begin{array}{l}\mathrm{C} 20 \\
119.8(5)\end{array}$ & $\mathrm{C} 21$ & $\mathrm{H} 21$ & $119.8(5)$ & F413 & $\mathrm{C} 413$ & C414 \\
\hline $\begin{array}{l}\text { C22 } \\
119.6(4)\end{array}$ & $\mathrm{C} 21$ & $\mathrm{H} 21$ & $119.8(5)$ & C412 & $\mathrm{C} 413$ & C414 \\
\hline $\begin{array}{l}\mathrm{O} 22 \\
120.8(5)\end{array}$ & $\mathrm{C} 22$ & $\mathrm{C} 17$ & $124.7(4)$ & F414 & $\mathrm{C} 414$ & C415 \\
\hline $\begin{array}{l}\mathrm{O} 22 \\
120.9(4)\end{array}$ & $\mathrm{C} 22$ & $\mathrm{C} 21$ & $115.9(5)$ & F414 & C414 & C413 \\
\hline $\begin{array}{l}\mathrm{C} 17 \\
118.2(5)\end{array}$ & $\mathrm{C} 22$ & $\mathrm{C} 21$ & $119.3(5)$ & C415 & C414 & C413 \\
\hline $\begin{array}{l}\text { N30 } \\
119.3(5)\end{array}$ & $\mathrm{C} 31$ & $\mathrm{C} 32$ & $120.4(4)$ & F415 & $\mathrm{C} 415$ & C414 \\
\hline $\begin{array}{l}\text { N30 } \\
119.8(4)\end{array}$ & $\mathrm{C} 31$ & $\mathrm{C} 36$ & $118.6(4)$ & F415 & $\mathrm{C} 415$ & C416 \\
\hline $\begin{array}{l}\mathrm{C} 32 \\
120.9(5)\end{array}$ & $\mathrm{C} 31$ & $\mathrm{C} 36$ & $121.0(4)$ & C414 & $\mathrm{C} 415$ & C416 \\
\hline $\begin{array}{l}\text { C33 } \\
115.5(4)\end{array}$ & $\mathrm{C} 32$ & C31 & $118.5(4)$ & F416 & C416 & C415 \\
\hline $\begin{array}{l}\text { C33 } \\
120.5(4)\end{array}$ & $\mathrm{C} 32$ & C321 & $120.3(4)$ & F416 & $\mathrm{C} 416$ & C411 \\
\hline $\begin{array}{l}\mathrm{C} 31 \\
124.0(4)\end{array}$ & $\mathrm{C} 32$ & C321 & $121.2(4)$ & $\mathrm{C} 415$ & $\mathrm{C} 416$ & C411 \\
\hline $\begin{array}{l}\mathrm{C} 32 \\
113.0(4)\end{array}$ & $\mathrm{C} 33$ & $\mathrm{C} 34$ & $121.6(5)$ & $\mathrm{C} 426$ & $\mathrm{C} 421$ & C422 \\
\hline $\begin{array}{l}\mathrm{C} 32 \\
128.1(4)\end{array}$ & $\mathrm{C} 33$ & H33 & $119.2(5)$ & $\mathrm{C} 426$ & $\mathrm{C} 421$ & B4 \\
\hline $\begin{array}{l}\text { C34 } \\
118.5(4)\end{array}$ & $\mathrm{C} 33$ & H33 & $119.2(5)$ & $\mathrm{C} 422$ & $\mathrm{C} 421$ & B4 \\
\hline $\begin{array}{l}\text { C35 } \\
115.9(4)\end{array}$ & $\mathrm{C} 34$ & $\mathrm{C} 33$ & $118.8(5)$ & F422 & $\mathrm{C} 422$ & C423 \\
\hline $\begin{array}{l}\text { C35 } \\
118.9(4)\end{array}$ & C34 & C341 & $120.2(5)$ & F422 & $\mathrm{C} 422$ & C421 \\
\hline $\begin{array}{l}\mathrm{C} 33 \\
125.3(5)\end{array}$ & $\mathrm{C} 34$ & C341 & $121.0(5)$ & $\mathrm{C} 423$ & $\mathrm{C} 422$ & C421 \\
\hline $\begin{array}{l}\mathrm{C} 36 \\
120.6(4)\end{array}$ & $\mathrm{C} 35$ & C34 & $122.1(5)$ & F423 & $\mathrm{C} 423$ & C424 \\
\hline $\mathrm{C} 36$ & C35 & H35 & $119.0(5)$ & F423 & C423 & $\mathrm{C} 422$ \\
\hline
\end{tabular}




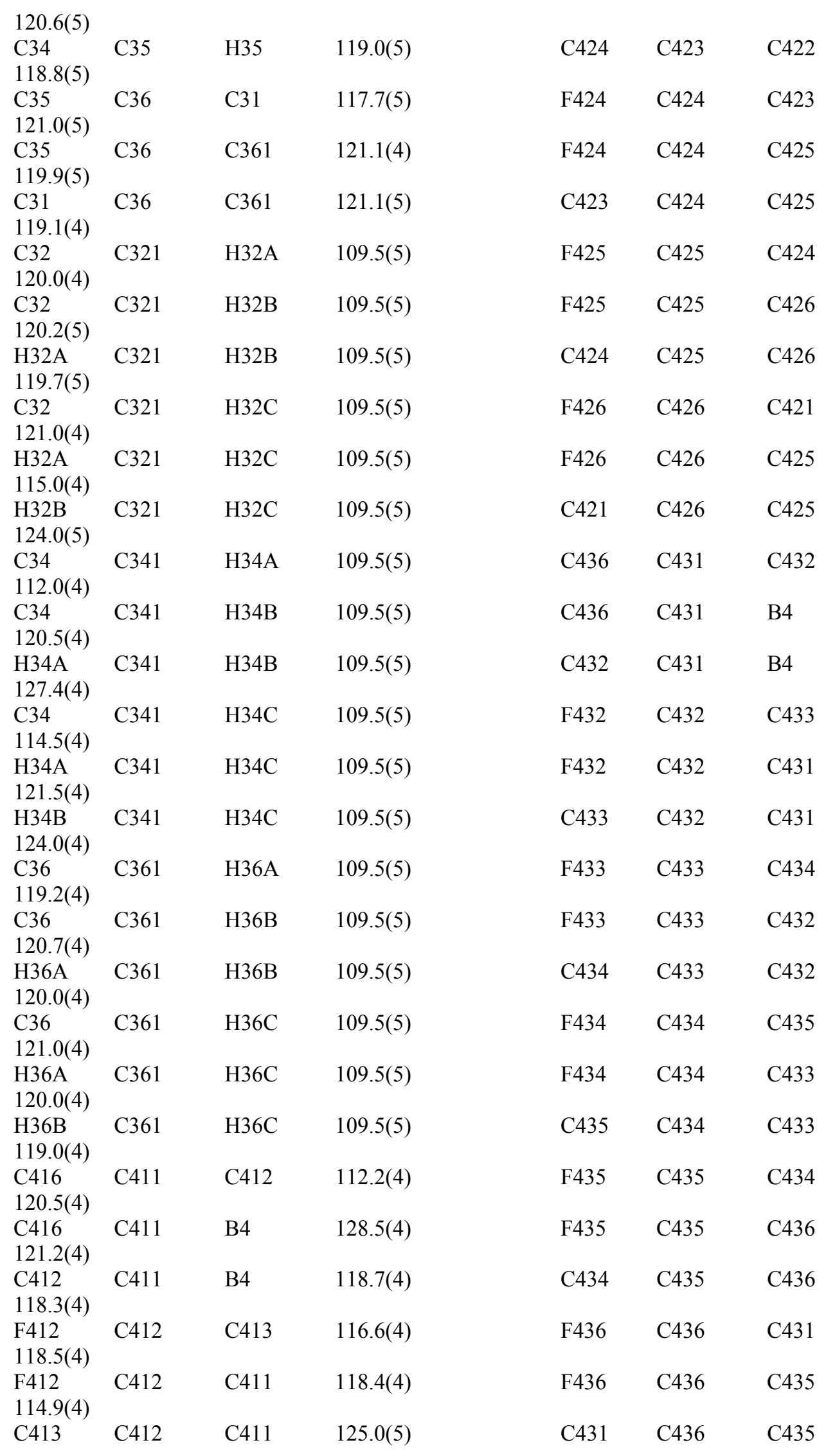




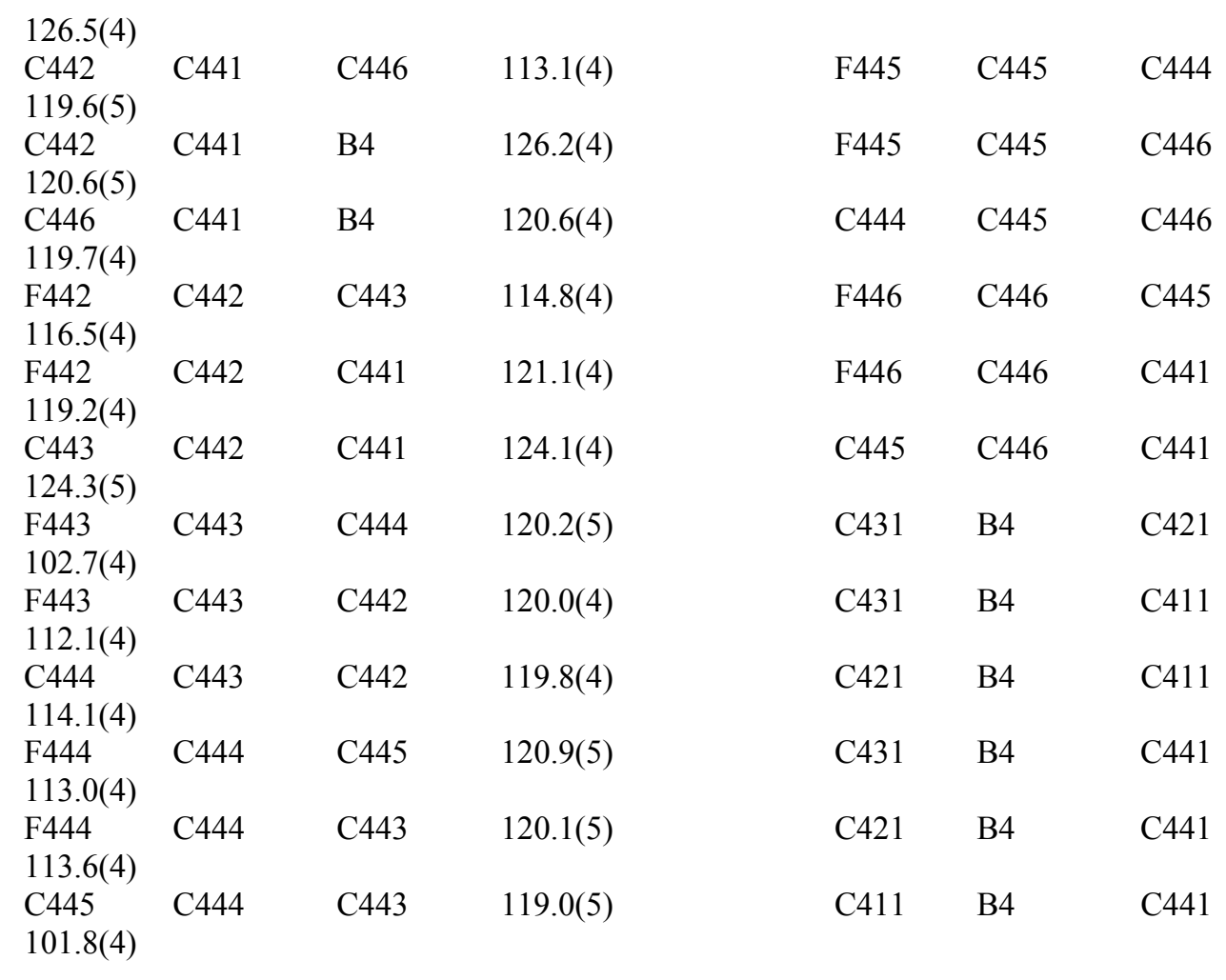

Numbers in parentheses are estimated standard deviations in the least significant digits

Table S2. Bond Distances in Angstroms for $\left[\operatorname{Re}(\mathrm{O})(\mathrm{NMes})_{\left.\text {saldach }^{+}\right], \mathbf{5 a}}\right.$

\begin{tabular}{|c|c|c|c|c|c|}
\hline Atom 1 & $\underline{\text { Atom }} \underline{2}$ & Distance & Atom 1 & $\underline{\text { Atom }} \underline{2}$ & Distance \\
\hline$\overline{\operatorname{Re}}-$ & $\overline{\mathrm{O}}$ & $\overline{1.714(3)}$ & $\overline{\mathrm{C} 10}$ & $\overline{\mathrm{C} 11}$ & $\overline{1.531(7)}$ \\
\hline $\mathrm{Re}$ & N30 & $1.751(4)$ & C10 & H10A & $0.990(7)$ \\
\hline $\mathrm{Re}$ & $\mathrm{O} 22$ & $1.914(3)$ & $\mathrm{C} 10$ & H10B & $0.990(7)$ \\
\hline $\mathrm{Re}$ & $\mathrm{O} 1$ & $2.052(3)$ & $\mathrm{C} 11$ & $\mathrm{C} 12$ & $1.514(7)$ \\
\hline $\operatorname{Re}$ & N8 & $2.070(4)$ & C11 & H11A & $0.990(7)$ \\
\hline $\mathrm{Re}$ & N15 & $2.192(4)$ & C11 & H11B & $0.990(7)$ \\
\hline F412 & $\mathrm{C} 412$ & $1.356(5)$ & $\mathrm{C} 12$ & $\mathrm{C} 13$ & $1.538(7)$ \\
\hline F413 & C413 & $1.347(5)$ & $\mathrm{C} 12$ & $\mathrm{H} 12 \mathrm{~A}$ & $0.990(7)$ \\
\hline F414 & C414 & $1.335(6)$ & $\mathrm{C} 12$ & H12B & $0.990(7)$ \\
\hline F415 & C415 & $1.356(6)$ & $\mathrm{C} 13$ & C14 & $1.525(6)$ \\
\hline F416 & $\mathrm{C} 416$ & $1.360(5)$ & $\mathrm{C} 13$ & H13A & $0.990(6)$ \\
\hline F422 & C422 & $1.363(5)$ & $\mathrm{C} 13$ & H13B & $0.990(6)$ \\
\hline F423 & $\mathrm{C} 423$ & $1.341(6)$ & $\mathrm{C} 14$ & H14 & $1.000(6)$ \\
\hline F424 & $\mathrm{C} 424$ & $1.343(5)$ & $\mathrm{C} 16$ & $\mathrm{C} 17$ & $1.439(7)$ \\
\hline F425 & $\mathrm{C} 425$ & $1.342(5)$ & $\mathrm{C} 16$ & H16 & $0.950(7)$ \\
\hline F426 & $\mathrm{C} 426$ & $1.358(6)$ & $\mathrm{C} 17$ & $\mathrm{C} 22$ & $1.395(7)$ \\
\hline F432 & C432 & $1.348(5)$ & C17 & $\mathrm{C} 18$ & $1.396(7)$ \\
\hline F433 & $\mathrm{C} 433$ & $1.343(5)$ & $\mathrm{C} 18$ & C19 & $1.376(7)$ \\
\hline F434 & C434 & $1.344(5)$ & $\mathrm{C} 18$ & H18 & $0.950(7)$ \\
\hline F435 & C435 & $1.346(5)$ & C19 & $\mathrm{C} 20$ & $1.393(8)$ \\
\hline F436 & C436 & $1.359(5)$ & C19 & H19 & $0.950(8)$ \\
\hline F442 & $\mathrm{C} 442$ & $1.350(5)$ & $\mathrm{C} 20$ & $\mathrm{C} 21$ & $1.370(8)$ \\
\hline F443 & C443 & $1.355(5)$ & $\mathrm{C} 20$ & $\mathrm{H} 20$ & $0.950(8)$ \\
\hline
\end{tabular}




\begin{tabular}{|c|c|c|c|c|c|}
\hline F444 & C444 & $1.345(6)$ & $\mathrm{C} 21$ & $\mathrm{C} 22$ & $1.397(7)$ \\
\hline F445 & C445 & $1.354(5)$ & $\mathrm{C} 21$ & $\mathrm{H} 21$ & $0.950(7)$ \\
\hline F446 & $\mathrm{C} 446$ & $1.360(5)$ & $\mathrm{C} 31$ & $\mathrm{C} 32$ & $1.397(7)$ \\
\hline $\mathrm{O} 1$ & $\mathrm{C} 1$ & $1.316(6)$ & C31 & C36 & $1.428(6)$ \\
\hline $\mathrm{O} 22$ & $\mathrm{C} 22$ & $1.359(6)$ & C32 & C33 & $1.389(7)$ \\
\hline N8 & C7 & $1.297(6)$ & C32 & C 321 & $1.512(6)$ \\
\hline N8 & C9 & $1.477(6)$ & C33 & C34 & $1.392(7)$ \\
\hline N15 & $\mathrm{C} 16$ & $1.286(6)$ & C33 & H33 & $0.950(7)$ \\
\hline N15 & $\mathrm{C} 14$ & $1.487(5)$ & C34 & C35 & $1.391(7)$ \\
\hline N30 & C31 & $1.376(6)$ & $\mathrm{C} 34$ & C341 & $1.517(8)$ \\
\hline C1 & C2 & $1.412(7)$ & C35 & C36 & $1.382(7)$ \\
\hline $\mathrm{C} 1$ & C6 & $1.429(7)$ & C35 & H35 & $0.950(7)$ \\
\hline $\mathrm{C} 2$ & $\mathrm{C} 3$ & $1.360(8)$ & C36 & C361 & $1.489(7)$ \\
\hline C2 & $\mathrm{H} 2$ & $0.950(8)$ & C321 & $\mathrm{H} 32 \mathrm{~A}$ & $0.980(7)$ \\
\hline C3 & $\mathrm{C} 4$ & $1.382(8)$ & C 321 & H32B & $0.980(7)$ \\
\hline C3 & H3 & $0.950(8)$ & C321 & $\mathrm{H} 32 \mathrm{C}$ & $0.980(7)$ \\
\hline $\mathrm{C} 4$ & $\mathrm{C} 5$ & $1.380(8)$ & C341 & $\mathrm{H} 34 \mathrm{~A}$ & $0.980(7)$ \\
\hline C4 & $\mathrm{H} 4$ & $0.950(8)$ & C341 & H34B & $0.980(7)$ \\
\hline $\mathrm{C} 5$ & C6 & $1.424(7)$ & C341 & $\mathrm{H} 34 \mathrm{C}$ & $0.980(7)$ \\
\hline $\mathrm{C} 5$ & H5 & $0.950(7)$ & C361 & $\mathrm{H} 36 \mathrm{~A}$ & $0.980(7)$ \\
\hline C6 & C7 & $1.408(7)$ & C361 & H36B & $0.980(7)$ \\
\hline C7 & H7 & $0.950(7)$ & C361 & $\mathrm{H} 36 \mathrm{C}$ & $0.980(7)$ \\
\hline C9 & $\mathrm{C} 10$ & $1.518(7)$ & C411 & C416 & $1.395(7)$ \\
\hline C9 & $\mathrm{C} 14$ & $1.542(7)$ & C411 & $\mathrm{C} 412$ & $1.408(6)$ \\
\hline C9 & H9 & $1.000(7)$ & C411 & B4 & $1.654(8)$ \\
\hline C412 & C413 & $1.363(7)$ & C431 & B4 & $1.633(7)$ \\
\hline $\mathrm{C} 413$ & C414 & $1.380(7)$ & $\mathrm{C} 432$ & $\mathrm{C} 433$ & $1.383(6)$ \\
\hline C414 & C415 & $1.366(7)$ & C433 & C434 & $1.378(7)$ \\
\hline C415 & C416 & $1.370(7)$ & C434 & C435 & $1.373(7)$ \\
\hline C421 & C426 & $1.370(6)$ & C435 & C436 & $1.392(6)$ \\
\hline C421 & C422 & $1.393(7)$ & C441 & C442 & $1.384(6)$ \\
\hline C421 & B4 & $1.649(7)$ & C441 & C446 & $1.387(6)$ \\
\hline $\mathrm{C} 422$ & $\mathrm{C} 423$ & $1.373(7)$ & C441 & B4 & $1.654(7)$ \\
\hline C423 & C424 & $1.366(7)$ & C442 & C443 & $1.381(7)$ \\
\hline $\mathrm{C} 424$ & $\mathrm{C} 425$ & $1.371(8)$ & C443 & $\mathrm{C} 444$ & $1.362(7)$ \\
\hline C425 & C426 & $1.388(7)$ & C444 & C445 & $1.361(7)$ \\
\hline C431 & C436 & $1.371(6)$ & C445 & C446 & $1.378(7)$ \\
\hline C431 & C432 & $1.410(6)$ & & & \\
\hline
\end{tabular}

Numbers in parentheses are estimated standard deviations in the least significant digits.

Table S3. Torsional Angles in Degrees for $\left[\operatorname{Re}(\mathrm{O})\left(\mathrm{NMes}_{\mathrm{s}} \mathrm{saldach}^{+}\right], \mathbf{5 a}\right.$

\begin{tabular}{lllll}
$\underline{\text { Atom }} \underline{1}$ & $\underline{\text { Atom }} \underline{\underline{\mathrm{O}}}$ & $\underline{\underline{\text { Atom }}} \underline{\underline{3}}$ & $\underline{\text { Atom }} \underline{4}$ & \multicolumn{1}{c}{ Angle } \\
$\mathrm{O}(22)$ & $\mathrm{Re}$ & $\mathrm{O}(1)$ & $\mathrm{C}(1)$ & $-100.49(0.57)$ \\
$\mathrm{N}(8)$ & $\mathrm{Re}$ & $\mathrm{O}(1)$ & $\mathrm{C}(1)$ & $-44.38(0.38)$ \\
$\mathrm{N}(15)$ & $\mathrm{Re}$ & $\mathrm{O}(1)$ & $\mathrm{C}(1)$ & $-119.42(0.38)$ \\
$\mathrm{N}(30)$ & $\mathrm{Re}$ & $\mathrm{O}(1)$ & $\mathrm{C}(1)$ & $49.40(0.39)$ \\
$\mathrm{O}$ & $\mathrm{Re}$ & $\mathrm{O}(22)$ & $\mathrm{C}(22)$ & $-89.67(0.43)$ \\
$\mathrm{O}(1)$ & $\mathrm{Re}$ & $\mathrm{O}(22)$ & $\mathrm{C}(22)$ & $74.14(0.42)$ \\
$\mathrm{N}(8)$ & $\mathrm{Re}$ & $\mathrm{O}(22)$ & $\mathrm{C}(22)$ & $29.60(0.63)$ \\
$\mathrm{N}(15)$ & $\mathrm{Re}$ & $\mathrm{O}(22)$ & $\mathrm{C}(22)$ & $-8.12(0.42)$ \\
$\mathrm{N}(30)$ & $\mathrm{Re}$ & $\mathrm{O}(22)$ & $\mathrm{C}(22)$ & $165.93(0.42)$ \\
$\mathrm{O}$ & $\mathrm{Re}$ & $\mathrm{N}(8)$ & $\mathrm{C}(7)$ & $-158.28(0.43)$ \\
$\mathrm{O}$ & $\mathrm{Re}$ & $\mathrm{N}(8)$ & $\mathrm{C}(9)$ & $38.94(0.31)$
\end{tabular}




\begin{tabular}{|c|c|c|c|c|}
\hline $\mathrm{O}(1)$ & $\operatorname{Re}$ & $\mathrm{N}(8)$ & $C(7)$ & $35.47(0.41)$ \\
\hline $\mathrm{O}(1)$ & $\operatorname{Re}$ & $\mathrm{N}(8)$ & $\mathrm{C}(9)$ & $-127.31(0.31)$ \\
\hline $\mathrm{O}(22)$ & $\operatorname{Re}$ & $\mathrm{N}(8)$ & $C(7)$ & $80.28(0.59)$ \\
\hline $\mathrm{O}(22)$ & $\operatorname{Re}$ & $\mathrm{N}(8)$ & $\mathrm{C}(9)$ & $-82.49(0.46)$ \\
\hline $\mathrm{N}(15)$ & $\operatorname{Re}$ & $\mathrm{N}(8)$ & $C(7)$ & $119.68(0.44)$ \\
\hline $\mathrm{N}(15)$ & $\operatorname{Re}$ & $\mathrm{N}(8)$ & $\mathrm{C}(9)$ & $-43.10(0.30)$ \\
\hline $\mathrm{N}(30)$ & $\operatorname{Re}$ & $\mathrm{N}(8)$ & $C(7)$ & $-57.37(0.44)$ \\
\hline $\mathrm{N}(30)$ & $\operatorname{Re}$ & $\mathrm{N}(8)$ & $C(9)$ & $139.85(0.32)$ \\
\hline $\mathrm{O}$ & $\operatorname{Re}$ & $\mathrm{N}(15)$ & $\mathrm{C}(14)$ & $-73.97(0.31)$ \\
\hline $\mathrm{O}$ & $\operatorname{Re}$ & $\mathrm{N}(15)$ & $C(16)$ & $106.70(0.43)$ \\
\hline $\mathrm{O}(1)$ & $\operatorname{Re}$ & $\mathrm{N}(15)$ & $\mathrm{C}(14)$ & $100.67(0.31)$ \\
\hline $\mathrm{O}(1)$ & $\operatorname{Re}$ & $\mathrm{N}(15)$ & $C(16)$ & $-78.67(0.42)$ \\
\hline $\mathrm{O}(22)$ & $\operatorname{Re}$ & $\mathrm{N}(15)$ & $C(14)$ & $-176.49(0.32)$ \\
\hline $\mathrm{O}(22)$ & $\operatorname{Re}$ & $\mathrm{N}(15)$ & $C(16)$ & $4.17(0.42)$ \\
\hline $\mathrm{N}(8)$ & $\operatorname{Re}$ & $\mathrm{N}(15)$ & $\mathrm{C}(14)$ & $18.20(0.30)$ \\
\hline $\mathrm{N}(8)$ & $\operatorname{Re}$ & $\mathrm{N}(15)$ & $C(16)$ & $-161.14(0.45)$ \\
\hline $\mathrm{N}(30)$ & $\operatorname{Re}$ & $\mathrm{N}(15)$ & $C(14)$ & $32.47(0.99)$ \\
\hline $\mathrm{N}(30)$ & $\operatorname{Re}$ & $\mathrm{N}(15)$ & $C(16)$ & $-146.87(0.78)$ \\
\hline $\mathrm{O}$ & $\operatorname{Re}$ & $\mathrm{N}(30)$ & $\mathrm{C}(31)$ & $56.04(1.35)$ \\
\hline $\mathrm{O}(1)$ & $\operatorname{Re}$ & $\mathrm{N}(30)$ & $\mathrm{C}(31)$ & $-115.58(1.33)$ \\
\hline $\mathrm{O}(22)$ & $\operatorname{Re}$ & $\mathrm{N}(30)$ & $\mathrm{C}(31)$ & $161.30(1.31)$ \\
\hline $\mathrm{N}(8)$ & $\operatorname{Re}$ & $\mathrm{N}(30)$ & $\mathrm{C}(31)$ & $-34.74(1.34)$ \\
\hline $\mathrm{N}(15)$ & $\operatorname{Re}$ & $\mathrm{N}(30)$ & $\mathrm{C}(31)$ & $-48.49(1.84)$ \\
\hline $\operatorname{Re}$ & $\mathrm{O}(1)$ & $\mathrm{C}(1)$ & $\mathrm{C}(2)$ & $-153.86(0.38)$ \\
\hline $\operatorname{Re}$ & $\mathrm{O}(1)$ & $\mathrm{C}(1)$ & $\mathrm{C}(6)$ & $28.79(0.63)$ \\
\hline $\operatorname{Re}$ & $\mathrm{O}(22)$ & $C(22)$ & $\mathrm{C}(17)$ & $7.21(0.76)$ \\
\hline $\operatorname{Re}$ & $\mathrm{O}(22)$ & $\mathrm{C}(22)$ & $\mathrm{C}(21)$ & $-174.02(0.34)$ \\
\hline $\operatorname{Re}$ & $\mathrm{N}(8)$ & $C(7)$ & $C(6)$ & $-11.46(0.72)$ \\
\hline $\mathrm{C}(9)$ & $\mathrm{N}(8)$ & $C(7)$ & $\mathrm{C}(6)$ & $149.60(0.47)$ \\
\hline $\operatorname{Re}$ & $\mathrm{N}(8)$ & $\mathrm{C}(9)$ & $C(10)$ & $-178.34(0.36)$ \\
\hline $\operatorname{Re}$ & $\mathrm{N}(8)$ & $\mathrm{C}(9)$ & $\mathrm{C}(14)$ & $59.67(0.40)$ \\
\hline$C(7)$ & $\mathrm{N}(8)$ & $\mathrm{C}(9)$ & $C(10)$ & $17.79(0.67)$ \\
\hline$C(7)$ & $\mathrm{N}(8)$ & $C(9)$ & $C(14)$ & $-104.20(0.51)$ \\
\hline $\operatorname{Re}$ & $\mathrm{N}(15)$ & $\mathrm{C}(14)$ & $\mathrm{C}(9)$ & $8.29(0.44)$ \\
\hline $\operatorname{Re}$ & $\mathrm{N}(15)$ & $\mathrm{C}(14)$ & $\mathrm{C}(13)$ & $131.52(0.36)$ \\
\hline$C(16)$ & $\mathrm{N}(15)$ & $C(14)$ & $\mathrm{C}(9)$ & $-172.34(0.43)$ \\
\hline$C(16)$ & $\mathrm{N}(15)$ & $\mathrm{C}(14)$ & $\mathrm{C}(13)$ & $-49.11(0.62)$ \\
\hline $\operatorname{Re}$ & $\mathrm{N}(15)$ & $C(16)$ & $\mathrm{C}(17)$ & $0.75(0.74)$ \\
\hline$C(14)$ & $\mathrm{N}(15)$ & $C(16)$ & $\mathrm{C}(17)$ & $-178.55(0.47)$ \\
\hline $\mathrm{Re}$ & $\mathrm{N}(30)$ & $C(31)$ & $\mathrm{C}(32)$ & $-38.60(1.59)$ \\
\hline $\operatorname{Re}$ & $\mathrm{N}(30)$ & $\mathrm{C}(31)$ & $C(36)$ & $140.88(1.16)$ \\
\hline $\mathrm{O}(1)$ & $\mathrm{C}(1)$ & $\mathrm{C}(2)$ & $\mathrm{C}(3)$ & $179.22(0.52)$ \\
\hline$C(6)$ & $\mathrm{C}(1)$ & $\mathrm{C}(2)$ & $\mathrm{C}(3)$ & $-3.31(0.81)$ \\
\hline $\mathrm{O}(1)$ & $\mathrm{C}(1)$ & $C(6)$ & $C(5)$ & $178.14(0.47)$ \\
\hline $\mathrm{O}(1)$ & $\mathrm{C}(1)$ & $C(6)$ & $\mathrm{C}(7)$ & $13.62(0.71)$ \\
\hline $\mathrm{C}(2)$ & $\mathrm{C}(1)$ & $C(6)$ & $\mathrm{C}(5)$ & $0.76(0.72)$ \\
\hline$C(2)$ & $\mathrm{C}(1)$ & $C(6)$ & $C(7)$ & $-163.77(0.48)$ \\
\hline $\mathrm{C}(1)$ & $\mathrm{C}(2)$ & $\mathrm{C}(3)$ & $\mathrm{C}(4)$ & $3.26(0.94)$ \\
\hline$C(2)$ & $C(3)$ & $C(4)$ & $C(5)$ & $-0.51(0.96)$ \\
\hline$C(3)$ & $\mathrm{C}(4)$ & $C(5)$ & $\mathrm{C}(6)$ & $-2.05(0.90)$ \\
\hline $\mathrm{C}(4)$ & $C(5)$ & $C(6)$ & $\mathrm{C}(1)$ & $1.88(0.81)$ \\
\hline C(4) & $C(5)$ & $C(6)$ & $C(7)$ & $166.25(0.52)$ \\
\hline $\mathrm{C}(1)$ & $C(6)$ & $C(7)$ & $\mathrm{N}(8)$ & $-22.61(0.74)$ \\
\hline$C(5)$ & $C(6)$ & $C(7)$ & $\mathrm{N}(8)$ & $172.95(0.50)$ \\
\hline $\mathrm{N}(8)$ & $\mathrm{C}(9)$ & $C(10)$ & $\mathrm{C}(11)$ & $178.01(0.43)$ \\
\hline$C(14)$ & $\mathrm{C}(9)$ & $\mathrm{C}(10)$ & $\mathrm{C}(11)$ & $-63.02(0.54)$ \\
\hline
\end{tabular}




\begin{tabular}{|c|c|c|c|c|}
\hline $\mathrm{N}(8)$ & $C(9)$ & $C(14)$ & $\mathrm{N}(15)$ & $-41.10(0.48)$ \\
\hline $\mathrm{N}(8)$ & $C(9)$ & $C(14)$ & $C(13)$ & $-168.26(0.38)$ \\
\hline$C(10)$ & $C(9)$ & $C(14)$ & $\mathrm{N}(15)$ & $-168.15(0.40)$ \\
\hline$C(10)$ & $\mathrm{C}(9)$ & $C(14)$ & $\mathrm{C}(13)$ & $64.69(0.52)$ \\
\hline C(9) & $\mathrm{C}(10)$ & $\mathrm{C}(11)$ & $C(12)$ & $57.44(0.61)$ \\
\hline$C(10)$ & $C(11)$ & $C(12)$ & $C(13)$ & $-53.87(0.64)$ \\
\hline$C(11)$ & $\mathrm{C}(12)$ & $\mathrm{C}(13)$ & $\mathrm{C}(14)$ & $52.79(0.58)$ \\
\hline$C(12)$ & $\mathrm{C}(13)$ & $C(14)$ & $\mathrm{N}(15)$ & $-179.88(0.44)$ \\
\hline$C(12)$ & $\mathrm{C}(13)$ & $C(14)$ & $\mathrm{C}(9)$ & $-56.98(0.54)$ \\
\hline $\mathrm{N}(15)$ & $C(16)$ & $C(17)$ & $C(18)$ & $177.47(0.50)$ \\
\hline $\mathrm{N}(15)$ & $C(16)$ & $\mathrm{C}(17)$ & $\mathrm{C}(22)$ & $-4.60(0.86)$ \\
\hline$C(16)$ & $\mathrm{C}(17)$ & $\mathrm{C}(18)$ & $\mathrm{C}(19)$ & $178.78(0.51)$ \\
\hline$C(22)$ & $\mathrm{C}(17)$ & $\mathrm{C}(18)$ & $C(19)$ & $0.75(0.81)$ \\
\hline$C(16)$ & $\mathrm{C}(17)$ & $\mathrm{C}(22)$ & $\mathrm{O}(22)$ & $0.95(0.84)$ \\
\hline$C(16)$ & $\mathrm{C}(17)$ & $\mathrm{C}(22)$ & $\mathrm{C}(21)$ & $-177.78(0.50)$ \\
\hline$C(18)$ & $\mathrm{C}(17)$ & $\mathrm{C}(22)$ & $\mathrm{O}(22)$ & $178.82(0.48)$ \\
\hline$C(18)$ & $\mathrm{C}(17)$ & $\mathrm{C}(22)$ & $\mathrm{C}(21)$ & $0.10(0.80)$ \\
\hline$C(17)$ & $\mathrm{C}(18)$ & $C(19)$ & $C(20)$ & $-1.06(0.85)$ \\
\hline$C(18)$ & $\mathrm{C}(19)$ & $C(20)$ & $\mathrm{C}(21)$ & $0.52(0.86)$ \\
\hline$C(19)$ & $\mathrm{C}(20)$ & $\mathrm{C}(21)$ & $C(22)$ & $0.33(0.82)$ \\
\hline $\mathrm{C}(20)$ & $\mathrm{C}(21)$ & $\mathrm{C}(22)$ & $\mathrm{O}(22)$ & $-179.46(0.47)$ \\
\hline$C(20)$ & $\mathrm{C}(21)$ & $\mathrm{C}(22)$ & $\mathrm{C}(17)$ & $-0.63(0.79)$ \\
\hline $\mathrm{N}(30)$ & $\mathrm{C}(31)$ & $C(32)$ & $\mathrm{C}(33)$ & $175.73(0.46)$ \\
\hline $\mathrm{N}(30)$ & $\mathrm{C}(31)$ & $C(32)$ & $C(321)$ & $-2.44(0.76)$ \\
\hline$C(36)$ & $\mathrm{C}(31)$ & $\mathrm{C}(32)$ & $C(33)$ & $-3.74(0.77)$ \\
\hline C(36) & $\mathrm{C}(31)$ & $C(32)$ & $C(321)$ & $178.10(0.52)$ \\
\hline $\mathrm{N}(30)$ & $\mathrm{C}(31)$ & $C(36)$ & $C(35)$ & $-176.80(0.51)$ \\
\hline $\mathrm{N}(30)$ & $C(31)$ & $C(36)$ & $C(361)$ & $3.96(0.83)$ \\
\hline $\mathrm{C}(32)$ & $\mathrm{C}(31)$ & $C(36)$ & $C(35)$ & $2.68(0.83)$ \\
\hline C(32) & $\mathrm{C}(31)$ & $C(36)$ & $C(361)$ & $-176.57(0.55)$ \\
\hline $\mathrm{C}(31)$ & $\mathrm{C}(32)$ & $\mathrm{C}(33)$ & $\mathrm{C}(34)$ & 0.78 ( 0.77) \\
\hline$C(321)$ & $C(32)$ & $C(33)$ & $C(34)$ & $178.96(0.50)$ \\
\hline$C(32)$ & $C(33)$ & $C(34)$ & $C(35)$ & $3.16(0.80)$ \\
\hline$C(32)$ & $\mathrm{C}(33)$ & $C(34)$ & $C(341)$ & $-176.93(0.53)$ \\
\hline C(33) & $\mathrm{C}(34)$ & $C(35)$ & $\mathrm{C}(36)$ & $-4.28(0.88)$ \\
\hline$C(341)$ & $C(34)$ & $C(35)$ & $C(36)$ & $175.81(0.59)$ \\
\hline $\mathrm{C}(34)$ & $C(35)$ & $C(36)$ & $\mathrm{C}(31)$ & 1.41 ( 0.91) \\
\hline C(34) & $C(35)$ & $C(36)$ & $C(361)$ & $-179.35(0.59)$ \\
\hline$C(416)$ & $\mathrm{C}(411)$ & $C(412)$ & $F(412)$ & $179.96(0.39)$ \\
\hline$C(416)$ & $C(411)$ & $C(412)$ & $C(413)$ & $-0.20(0.73)$ \\
\hline $\mathrm{B}(4)$ & $C(411)$ & $C(412)$ & $F(412)$ & $8.25(0.66)$ \\
\hline B(4) & $\mathrm{C}(411)$ & $C(412)$ & $C(413)$ & $-171.91(0.47)$ \\
\hline$C(412)$ & $\mathrm{C}(411)$ & $C(416)$ & $F(416)$ & $-178.20(0.43)$ \\
\hline$C(412)$ & $C(411)$ & $C(416)$ & $C(415)$ & $0.60(0.72)$ \\
\hline B(4) & $\mathrm{C}(411)$ & $C(416)$ & $F(416)$ & $-7.49(0.78)$ \\
\hline $\mathrm{B}(4)$ & $\mathrm{C}(411)$ & $C(416)$ & $C(415)$ & $171.31(0.48)$ \\
\hline$C(412)$ & $\mathrm{C}(411)$ & $\mathrm{B}(4)$ & $C(421)$ & $-63.43(0.58)$ \\
\hline$C(412)$ & $C(411)$ & $\mathrm{B}(4)$ & $C(431)$ & $-179.57(0.42)$ \\
\hline$C(412)$ & $C(411)$ & $\mathrm{B}(4)$ & $C(441)$ & $59.28(0.53)$ \\
\hline$C(416)$ & $\mathrm{C}(411)$ & $\mathrm{B}(4)$ & $C(421)$ & $126.37(0.53)$ \\
\hline$C(416)$ & $\mathrm{C}(411)$ & $\mathrm{B}(4)$ & $C(431)$ & $10.23(0.70)$ \\
\hline$C(416)$ & $\mathrm{C}(411)$ & B(4) & $C(441)$ & $-110.92(0.54)$ \\
\hline$F(412)$ & $\mathrm{C}(412)$ & $C(413)$ & $\mathrm{F}(413)$ & $-0.17(0.73)$ \\
\hline$F(412)$ & $C(412)$ & $C(413)$ & $C(414)$ & $-179.83(0.43)$ \\
\hline$C(411)$ & $\mathrm{C}(412)$ & $C(413)$ & $F(413)$ & $180.00(0.65)$ \\
\hline$C(411)$ & $C(412)$ & $C(413)$ & $C(414)$ & $0.32(0.79)$ \\
\hline
\end{tabular}




\begin{tabular}{|c|c|c|c|c|}
\hline $\mathrm{F}(413)$ & $C(413)$ & $\mathrm{C}(414)$ & $F(414)$ & $0.21(0.73)$ \\
\hline$F(413)$ & $C(413)$ & $C(414)$ & $C(415)$ & $179.54(0.45)$ \\
\hline$C(412)$ & $C(413)$ & $C(414)$ & $F(414)$ & $179.88(0.46)$ \\
\hline$C(412)$ & $C(413)$ & $C(414)$ & $C(415)$ & $-0.79(0.74)$ \\
\hline$F(414)$ & $C(414)$ & $C(415)$ & $\mathrm{F}(415)$ & $2.18(0.72)$ \\
\hline$F(414)$ & $C(414)$ & $C(415)$ & $C(416)$ & $-179.49(0.45)$ \\
\hline$C(413)$ & $\mathrm{C}(414)$ & $C(415)$ & $\mathrm{F}(415)$ & $-177.14(0.45)$ \\
\hline$C(413)$ & $C(414)$ & $C(415)$ & $C(416)$ & 1.18 ( 0.75) \\
\hline$F(415)$ & $C(415)$ & $C(416)$ & $F(416)$ & $-3.96(0.69)$ \\
\hline$F(415)$ & $C(415)$ & $C(416)$ & $\mathrm{C}(411)$ & $177.18(0.46)$ \\
\hline$C(414)$ & $C(415)$ & $C(416)$ & $F(416)$ & $177.72(0.45)$ \\
\hline$C(414)$ & $C(415)$ & $C(416)$ & $C(411)$ & $-1.14(0.80)$ \\
\hline$C(426)$ & $C(421)$ & $C(422)$ & $\mathrm{F}(422)$ & $-178.71(0.38)$ \\
\hline$C(426)$ & $C(421)$ & $C(422)$ & $C(423)$ & 0.98 ( 0.68) \\
\hline B(4) & $\mathrm{C}(421)$ & $C(422)$ & $F(422)$ & $-5.53(0.62)$ \\
\hline B(4) & $C(421)$ & $C(422)$ & $C(423)$ & $174.16(0.46)$ \\
\hline$C(422)$ & $C(421)$ & $C(426)$ & $F(426)$ & $177.35(0.38)$ \\
\hline$C(422)$ & $\mathrm{C}(421)$ & $C(426)$ & $C(425)$ & $-2.07(0.68)$ \\
\hline B(4) & $C(421)$ & $C(426)$ & $F(426)$ & $4.97(0.71)$ \\
\hline $\mathrm{B}(4)$ & $C(421)$ & $C(426)$ & $C(425)$ & $-174.45(0.48)$ \\
\hline$C(422)$ & $C(421)$ & $\mathrm{B}(4)$ & $C(411)$ & $177.67(0.40)$ \\
\hline$C(422)$ & $C(421)$ & $\mathrm{B}(4)$ & $C(431)$ & $-60.77(0.51)$ \\
\hline$C(422)$ & $C(421)$ & $\mathrm{B}(4)$ & $\mathrm{C}(441)$ & $61.63(0.56)$ \\
\hline$C(426)$ & $C(421)$ & $\mathrm{B}(4)$ & $C(411)$ & $-10.31(0.68)$ \\
\hline$C(426)$ & $C(421)$ & $\mathrm{B}(4)$ & $C(431)$ & $111.26(0.51)$ \\
\hline$C(426)$ & $C(421)$ & $\mathrm{B}(4)$ & $C(441)$ & $-126.34(0.50)$ \\
\hline$F(422)$ & $C(422)$ & $C(423)$ & $\mathrm{F}(423)$ & $0.94(0.67)$ \\
\hline $\mathrm{F}(422)$ & $C(422)$ & $C(423)$ & $C(424)$ & $179.21(0.44)$ \\
\hline$C(421)$ & $C(422)$ & $C(423)$ & $\mathrm{F}(423)$ & $-178.76(0.43)$ \\
\hline$C(421)$ & $C(422)$ & $C(423)$ & $C(424)$ & $-0.48(0.79)$ \\
\hline$F(423)$ & $C(423)$ & $C(424)$ & $\mathrm{F}(424)$ & $-0.35(0.80)$ \\
\hline$F(423)$ & $C(423)$ & $C(424)$ & $C(425)$ & $179.23(0.49)$ \\
\hline$C(422)$ & $C(423)$ & $C(424)$ & $\mathrm{F}(424)$ & $-178.62(0.46)$ \\
\hline$C(422)$ & $C(423)$ & $C(424)$ & $C(425)$ & $0.96(0.80)$ \\
\hline$F(424)$ & $C(424)$ & $C(425)$ & $\mathrm{F}(425)$ & $0.50(0.80)$ \\
\hline $\mathrm{F}(424)$ & $C(424)$ & $C(425)$ & $C(426)$ & $177.59(0.46)$ \\
\hline$C(423)$ & $C(424)$ & $C(425)$ & $\mathrm{F}(425)$ & $-179.08(0.48)$ \\
\hline$C(423)$ & $C(424)$ & $C(425)$ & $C(426)$ & $-2.00(0.82)$ \\
\hline$F(425)$ & $C(425)$ & $C(426)$ & $F(426)$ & $0.32(0.67)$ \\
\hline$F(425)$ & $C(425)$ & $C(426)$ & $C(421)$ & $179.78(0.44)$ \\
\hline$C(424)$ & $C(425)$ & $C(426)$ & $F(426)$ & $-176.76(0.46)$ \\
\hline$C(424)$ & $C(425)$ & $C(426)$ & $C(421)$ & $2.69(0.80)$ \\
\hline$C(436)$ & $\mathrm{C}(431)$ & $C(432)$ & $\mathrm{F}(432)$ & $174.17(0.39)$ \\
\hline$C(436)$ & $C(431)$ & $C(432)$ & $C(433)$ & $-3.73(0.64)$ \\
\hline B(4) & $C(431)$ & $C(432)$ & $F(432)$ & $-1.97(0.71)$ \\
\hline B(4) & $C(431)$ & $C(432)$ & $C(433)$ & $-179.88(0.47)$ \\
\hline$C(432)$ & $C(431)$ & $C(436)$ & $F(436)$ & $-178.26(0.38)$ \\
\hline$C(432)$ & $C(431)$ & $C(436)$ & $C(435)$ & 3.08 ( 0.67) \\
\hline $\mathrm{B}(4)$ & $C(431)$ & $C(436)$ & $F(436)$ & $-1.81(0.64)$ \\
\hline B(4) & $C(431)$ & $C(436)$ & $C(435)$ & $179.53(0.46)$ \\
\hline$C(432)$ & $\mathrm{C}(431)$ & $\mathrm{B}(4)$ & $C(411)$ & $-123.96(0.48)$ \\
\hline$C(432)$ & $C(431)$ & $\mathrm{B}(4)$ & $C(421)$ & $113.22(0.49)$ \\
\hline$C(432)$ & $C(431)$ & $\mathrm{B}(4)$ & $\mathrm{C}(441)$ & $-9.52(0.68)$ \\
\hline$C(436)$ & $C(431)$ & $\mathrm{B}(4)$ & $C(411)$ & $60.20(0.57)$ \\
\hline$C(436)$ & $C(431)$ & $\mathrm{B}(4)$ & $C(421)$ & $-62.63(0.53)$ \\
\hline$C(436)$ & $C(431)$ & $\mathrm{B}(4)$ & $C(441)$ & $174.63(0.41)$ \\
\hline
\end{tabular}




\begin{tabular}{|c|c|c|c|c|}
\hline$F(432)$ & $C(432)$ & $C(433)$ & $F(433)$ & $3.90(0.61)$ \\
\hline $\mathrm{F}(432)$ & $C(432)$ & $C(433)$ & $C(434)$ & $-175.81(0.40)$ \\
\hline C(431) & $C(432)$ & $C(433)$ & $F(433)$ & $-178.06(0.40)$ \\
\hline$C(431)$ & $C(432)$ & $C(433)$ & $C(434)$ & $2.22(0.70)$ \\
\hline $\mathrm{F}(433)$ & $C(433)$ & $C(434)$ & $\mathrm{F}(434)$ & $0.61(0.66)$ \\
\hline $\mathrm{F}(433)$ & $C(433)$ & $C(434)$ & $C(435)$ & $-179.33(0.41)$ \\
\hline C(432) & $C(433)$ & $C(434)$ & $F(434)$ & $-179.67(0.41)$ \\
\hline$C(432)$ & $C(433)$ & $C(434)$ & $C(435)$ & $0.39(0.69)$ \\
\hline $\mathrm{F}(434)$ & $C(434)$ & $C(435)$ & $F(435)$ & $-0.40(0.69)$ \\
\hline $\mathrm{F}(434)$ & $C(434)$ & $C(435)$ & $C(436)$ & $179.00(0.41)$ \\
\hline C(433) & $C(434)$ & $C(435)$ & $\mathrm{F}(435)$ & $179.54(0.42)$ \\
\hline$C(433)$ & $C(434)$ & $C(435)$ & $C(436)$ & $-1.06(0.69)$ \\
\hline$F(435)$ & $C(435)$ & $C(436)$ & $F(436)$ & $-0.15(0.63)$ \\
\hline$F(435)$ & $C(435)$ & $C(436)$ & $C(431)$ & $178.55(0.43)$ \\
\hline$C(434)$ & $C(435)$ & $C(436)$ & $F(436)$ & $-179.55(0.41)$ \\
\hline$C(434)$ & $C(435)$ & $C(436)$ & $C(431)$ & $-0.85(0.74)$ \\
\hline$C(446)$ & $C(441)$ & $C(442)$ & $F(442)$ & $-175.93(0.41)$ \\
\hline$C(446)$ & $\mathrm{C}(441)$ & $\mathrm{C}(442)$ & $C(443)$ & $1.60(0.70)$ \\
\hline $\mathrm{B}(4)$ & $C(441)$ & $C(442)$ & $F(442)$ & $0.43(0.71)$ \\
\hline B(4) & $C(441)$ & $C(442)$ & $C(443)$ & $177.96(0.49)$ \\
\hline$C(442)$ & $C(441)$ & $C(446)$ & $F(446)$ & $178.21(0.40)$ \\
\hline$C(442)$ & $\mathrm{C}(441)$ & $C(446)$ & $C(445)$ & $-2.00(0.71)$ \\
\hline $\mathrm{B}(4)$ & $C(441)$ & $C(446)$ & $F(446)$ & $1.62(0.67)$ \\
\hline B(4) & $C(441)$ & $C(446)$ & $C(445)$ & $-178.59(0.48)$ \\
\hline$C(442)$ & $\mathrm{C}(441)$ & $\mathrm{B}(4)$ & $C(411)$ & $-112.17(0.50)$ \\
\hline$C(442)$ & $\mathrm{C}(441)$ & $\mathrm{B}(4)$ & $C(421)$ & $10.86(0.68)$ \\
\hline$C(442)$ & $\mathrm{C}(441)$ & $\mathrm{B}(4)$ & $\mathrm{C}(431)$ & $127.30(0.49)$ \\
\hline$C(446)$ & $\mathrm{C}(441)$ & $\mathrm{B}(4)$ & $\mathrm{C}(411)$ & $63.94(0.53)$ \\
\hline$C(446)$ & $\mathrm{C}(441)$ & $\mathrm{B}(4)$ & $\mathrm{C}(421)$ & $-173.03(0.42)$ \\
\hline$C(446)$ & $\mathrm{C}(441)$ & $\mathrm{B}(4)$ & $\mathrm{C}(431)$ & $-56.59(0.60)$ \\
\hline $\mathrm{F}(442)$ & $C(442)$ & $C(443)$ & $F(443)$ & $-2.71(0.70)$ \\
\hline $\mathrm{F}(442)$ & $C(442)$ & $C(443)$ & $C(444)$ & $178.08(0.48)$ \\
\hline $\mathrm{C}(441)$ & $\mathrm{C}(442)$ & $\mathrm{C}(443)$ & $\mathrm{F}(443)$ & $179.62(0.45)$ \\
\hline C(441) & $C(442)$ & $\mathrm{C}(443)$ & $\mathrm{C}(444)$ & $0.41(0.83)$ \\
\hline $\mathrm{F}(443)$ & $C(443)$ & $\mathrm{C}(444)$ & $\mathrm{F}(444)$ & $-0.81(0.83)$ \\
\hline $\mathrm{F}(443)$ & $C(443)$ & $\mathrm{C}(444)$ & $C(445)$ & $178.63(0.51)$ \\
\hline$C(442)$ & $C(443)$ & $\mathrm{C}(444)$ & $\mathrm{F}(444)$ & $178.41(0.47)$ \\
\hline C(442) & $C(443)$ & $\mathrm{C}(444)$ & $C(445)$ & $-2.16(0.86)$ \\
\hline $\mathrm{F}(444)$ & $C(444)$ & $C(445)$ & $F(445)$ & $-0.11(0.84)$ \\
\hline $\mathrm{F}(444)$ & $C(444)$ & $C(445)$ & $C(446)$ & $-178.79(0.48)$ \\
\hline$C(443)$ & $\mathrm{C}(444)$ & $C(445)$ & $\mathrm{F}(445)$ & $-179.54(0.52)$ \\
\hline$C(443)$ & $C(444)$ & $C(445)$ & $C(446)$ & $1.78(0.86)$ \\
\hline $\mathrm{F}(445)$ & $C(445)$ & $C(446)$ & $\mathrm{F}(446)$ & $1.52(0.74)$ \\
\hline $\mathrm{F}(445)$ & $C(445)$ & $C(446)$ & $\mathrm{C}(441)$ & $-178.27(0.48)$ \\
\hline$C(444)$ & $\mathrm{C}(445)$ & $C(446)$ & $\mathrm{F}(446)$ & $-179.81(0.47)$ \\
\hline$C(444)$ & $C(445)$ & $C(446)$ & $C(441)$ & $0.39(0.84)$ \\
\hline
\end{tabular}


Table S4. Positional Parameters and Their Estimated Standard Deviations for $\left[\operatorname{Re}(\mathrm{O})(\mathrm{NMes})\right.$ saldach $^{+}$, 5a

\begin{tabular}{|c|c|c|c|c|}
\hline Atom & $\underline{x}$ & $\mathrm{y}$ & $\underline{\mathrm{Z}}$ & $\underline{\mathrm{U}}\left(\AA^{2}\right)$ \\
\hline$\overline{\operatorname{Re}}$ & $0 . \overline{1} 04292(16)$ & $0.343254(14)$ & $0 . \overline{2} 67345(14)$ & $0.03063(7)$ \\
\hline $\mathrm{F}(412)$ & $0.2932(2)$ & $0.22656(19)$ & $-0.34030(19)$ & $0.0368(10)$ \\
\hline$F(413)$ & $0.3344(3)$ & $0.1740(2)$ & $-0.5017(2)$ & $0.0463(11)$ \\
\hline$F(414)$ & $0.3889(2)$ & $-0.0261(2)$ & $-0.5778(2)$ & $0.0476(11)$ \\
\hline$F(415)$ & $0.4062(3)$ & $-0.1689(2)$ & $-0.4850(2)$ & $0.0477(11)$ \\
\hline$F(416)$ & $0.3579(3)$ & $-0.1226(2)$ & $-0.32785(19)$ & $0.0412(10)$ \\
\hline$F(422)$ & $0.2244(2)$ & $0.1969(2)$ & $-0.06466(19)$ & $0.0410(10)$ \\
\hline$F(423)$ & $0.3405(3)$ & $0.3496(2)$ & $0.0737(2)$ & $0.0512(11)$ \\
\hline$F(424)$ & $0.5370(3)$ & $0.4377(2)$ & $0.0649(2)$ & $0.0558(11)$ \\
\hline$F(425)$ & $0.6105(2)$ & $0.3669(2)$ & $-0.0869(2)$ & $0.0540(11)$ \\
\hline$F(426)$ & $0.4998(2)$ & $0.2115(2)$ & $-0.2239(2)$ & $0.0387(10)$ \\
\hline$F(432)$ & $0.1022(2)$ & $-0.0128(2)$ & $-0.1675(2)$ & $0.0373(10)$ \\
\hline $\mathrm{F}(433)$ & $0.1386(2)$ & $-0.1618(2)$ & $-0.08887(19)$ & $0.0398(10)$ \\
\hline $\mathrm{F}(434)$ & $0.3455(3)$ & $-0.2249(2)$ & $-0.0424(2)$ & $0.0463(11)$ \\
\hline$F(435)$ & $0.5209(2)$ & $-0.1342(2)$ & $-0.0812(2)$ & $0.0475(11)$ \\
\hline$F(436)$ & $0.4849(2)$ & $0.0106(2)$ & $-0.1672(2)$ & $0.0380(10)$ \\
\hline$F(442)$ & $0.1683(2)$ & $0.30335(19)$ & $-0.2012(2)$ & $0.0407(10)$ \\
\hline $\mathrm{F}(443)$ & $-0.0414(3)$ & $0.3274(2)$ & $-0.2662(2)$ & $0.0512(11)$ \\
\hline $\mathrm{F}(444)$ & $-0.1829(2)$ & $0.1585(2)$ & $-0.3722(2)$ & $0.0600(14)$ \\
\hline $\mathrm{F}(445)$ & $-0.1054(3)$ & $-0.0375(2)$ & $-0.4138(3)$ & $0.0658(14)$ \\
\hline $\mathrm{F}(446)$ & $0.1076(2)$ & $-0.06484(19)$ & $-0.3480(2)$ & $0.0425(10)$ \\
\hline $\mathrm{O}$ & $0.0247(3)$ & $0.2390(2)$ & $0.2707(2)$ & $0.0368(11)$ \\
\hline $\mathrm{O}(1)$ & $0.2158(3)$ & $0.4339(2)$ & $0.2372(2)$ & $0.0375(11)$ \\
\hline $\mathrm{O}(22)$ & $-0.0019(3)$ & $0.4094(2)$ & $0.1978(2)$ & $0.0366(11)$ \\
\hline $\mathrm{N}(8)$ & $0.2469(3)$ & $0.2672(3)$ & $0.2984(3)$ & $0.0308(12)$ \\
\hline $\mathrm{N}(15)$ & $0.1195(3)$ & $0.2313(3)$ & $0.1408(3)$ & $0.0270(12)$ \\
\hline $\mathrm{N}(30)$ & $0.1211(3)$ & $0.4273(3)$ & $0.3765(3)$ & $0.0310(14)$ \\
\hline $\mathrm{C}(1)$ & $0.3168(4)$ & $0.4698(4)$ & $0.2857(3)$ & $0.0369(17)$ \\
\hline$C(2)$ & $0.3658(5)$ & $0.5627(4)$ & $0.2791(4)$ & $0.052(2)$ \\
\hline$C(3)$ & $0.4695(5)$ & $0.6025(5)$ & $0.3291(4)$ & $0.062(2)$ \\
\hline C(4) & $0.5330(5)$ & $0.5515(5)$ & $0.3846(4)$ & $0.056(2)$ \\
\hline$C(5)$ & $0.4914(5)$ & $0.4581(4)$ & $0.3910(4)$ & $0.0472(18)$ \\
\hline$C(6)$ & $0.3815(4)$ & $0.4160(4)$ & $0.3426(3)$ & $0.0356(17)$ \\
\hline$C(7)$ & $0.3473(4)$ & $0.3111(4)$ & $0.3332(3)$ & $0.0351(17)$ \\
\hline C(9) & $0.2268(4)$ & $0.1527(3)$ & $0.2514(3)$ & $0.0321(16)$ \\
\hline$C(10)$ & $0.3214(5)$ & $0.0830(4)$ & $0.2680(4)$ & $0.0390(18)$ \\
\hline $\mathrm{C}(11)$ & $0.2776(5)$ & $-0.0310(4)$ & $0.2145(4)$ & $0.045(2)$ \\
\hline$C(12)$ & $0.2354(4)$ & $-0.0437(4)$ & $0.1159(4)$ & $0.0390(18)$ \\
\hline$C(13)$ & $0.1481(4)$ & $0.0340(3)$ & $0.0967(4)$ & $0.0377(17)$ \\
\hline $\mathrm{C}(14)$ & $0.1944(4)$ & $0.1462(3)$ & $0.1517(3)$ & $0.0299(16)$ \\
\hline$C(16)$ & $0.0709(4)$ & $0.2356(4)$ & $0.0624(3)$ & $0.0315(17)$ \\
\hline$C(17)$ & $-0.0012(4)$ & $0.3151(4)$ & $0.0433(3)$ & $0.0302(17)$ \\
\hline $\mathrm{C}(18)$ & $-0.0417(4)$ & $0.3073(4)$ & $-0.0467(4)$ & $0.0407(18)$ \\
\hline C(19) & $-0.1128(5)$ & $0.3784(4)$ & $-0.0717(4)$ & $0.047(2)$ \\
\hline$C(20)$ & $-0.1460(4)$ & $0.4580(4)$ & $-0.0062(4)$ & $0.044(2)$ \\
\hline $\mathrm{C}(21)$ & $-0.1077(4)$ & $0.4662(4)$ & $0.0824(4)$ & $0.041(2)$ \\
\hline$C(22)$ & $-0.0343(4)$ & $0.3952(4)$ & $0.1085(3)$ & $0.0324(17)$ \\
\hline$C(31)$ & $0.1455(4)$ & $0.4699(3)$ & $0.4680(3)$ & $0.0295(16)$ \\
\hline$C(32)$ & $0.1273(4)$ & $0.4088(4)$ & $0.5218(3)$ & $0.0327(17)$ \\
\hline$C(33)$ & $0.1600(4)$ & $0.4526(4)$ & $0.6139(3)$ & $0.0382(17)$ \\
\hline
\end{tabular}




\begin{tabular}{|c|c|c|c|c|}
\hline Atom & $\underline{x}$ & $\mathrm{y}$ & $\underline{\mathrm{z}}$ & $\underline{\mathrm{U}\left(\AA^{2}\right)}$ \\
\hline$C(35)$ & $0.2197(5)$ & $0.6169(4)$ & $0.5985(4)$ & $0.0437(19)$ \\
\hline$C(36)$ & $0.1905(5)$ & $0.5772(4)$ & $0.5064(4)$ & $0.0395(16)$ \\
\hline$C(321)$ & $0.0764(5)$ & $0.2963(4)$ & $0.4813(4)$ & $0.0439(19)$ \\
\hline$C(341)$ & $0.2492(6)$ & $0.6001(5)$ & $0.7539(4)$ & $0.061(2)$ \\
\hline$C(361)$ & $0.2041(6)$ & $0.6443(4)$ & $0.4491(4)$ & $0.066(3)$ \\
\hline$C(411)$ & $0.3243(4)$ & $0.0564(3)$ & $-0.3231(3)$ & $0.0287(16)$ \\
\hline$C(412)$ & $0.3199(4)$ & $0.1263(4)$ & $-0.3747(3)$ & $0.0331(17)$ \\
\hline C(413) & $0.3408(4)$ & $0.1014(4)$ & $-0.4576(3)$ & $0.0362(18)$ \\
\hline C(414) & $0.3682(4)$ & $0.0006(4)$ & $-0.4967(3)$ & $0.0364(18)$ \\
\hline$C(415)$ & $0.3747(4)$ & $-0.0708(4)$ & $-0.4497(3)$ & $0.0348(17)$ \\
\hline$C(416)$ & $0.3526(4)$ & $-0.0437(4)$ & $-0.3666(3)$ & $0.0323(17)$ \\
\hline$C(421)$ & $0.3537(4)$ & $0.1976(3)$ & $-0.1533(3)$ & $0.0269(14)$ \\
\hline$C(422)$ & $0.3208(4)$ & $0.2381(3)$ & $-0.0735(3)$ & $0.0310(16)$ \\
\hline$C(423)$ & $0.3784(4)$ & $0.3175(4)$ & $-0.0009(3)$ & $0.0356(17)$ \\
\hline C(424) & $0.4765(4)$ & $0.3613(4)$ & $-0.0051(4)$ & $0.0376(16)$ \\
\hline$C(425)$ & $0.5136(4)$ & $0.3259(4)$ & $-0.0819(4)$ & $0.037(2)$ \\
\hline$C(426)$ & $0.4530(4)$ & $0.2442(3)$ & $-0.1529(3)$ & $0.0309(16)$ \\
\hline$C(431)$ & $0.2922(4)$ & $0.0042(3)$ & $-0.1782(3)$ & $0.0273(14)$ \\
\hline C(432) & $0.2073(4)$ & $-0.0425(3)$ & $-0.1527(3)$ & $0.0284(14)$ \\
\hline$C(433)$ & $0.2241(4)$ & $-0.1186(3)$ & $-0.1088(3)$ & $0.0298(16)$ \\
\hline C(434) & $0.3294(4)$ & $-0.1505(4)$ & $-0.0847(3)$ & $0.0329(17)$ \\
\hline$C(435)$ & $0.4168(4)$ & $-0.1053(4)$ & $-0.1044(3)$ & $0.0329(17)$ \\
\hline$C(436)$ & $0.3945(4)$ & $-0.0300(3)$ & $-0.1495(3)$ & $0.0311(16)$ \\
\hline$C(441)$ & $0.1494(4)$ & $0.1166(3)$ & $-0.2672(3)$ & $0.0280(16)$ \\
\hline$C(442)$ & $0.1046(4)$ & $0.2140(3)$ & $-0.2511(3)$ & $0.0312(17)$ \\
\hline C(443) & $-0.0047(4)$ & $0.2290(4)$ & $-0.2861(4)$ & $0.0363(17)$ \\
\hline C(444) & $-0.0757(4)$ & $0.1446(4)$ & $-0.3397(4)$ & $0.043(2)$ \\
\hline$C(445)$ & $-0.0360(4)$ & $0.0470(4)$ & $-0.3601(4)$ & $0.041(2)$ \\
\hline$C(446)$ & $0.0737(4)$ & $0.0348(4)$ & $-0.3245(3)$ & $0.0348(17)$ \\
\hline$B(4)$ & $0.2803(4)$ & $0.0943(4)$ & $-0.2298(4)$ & $0.0292(17)$ \\
\hline
\end{tabular}




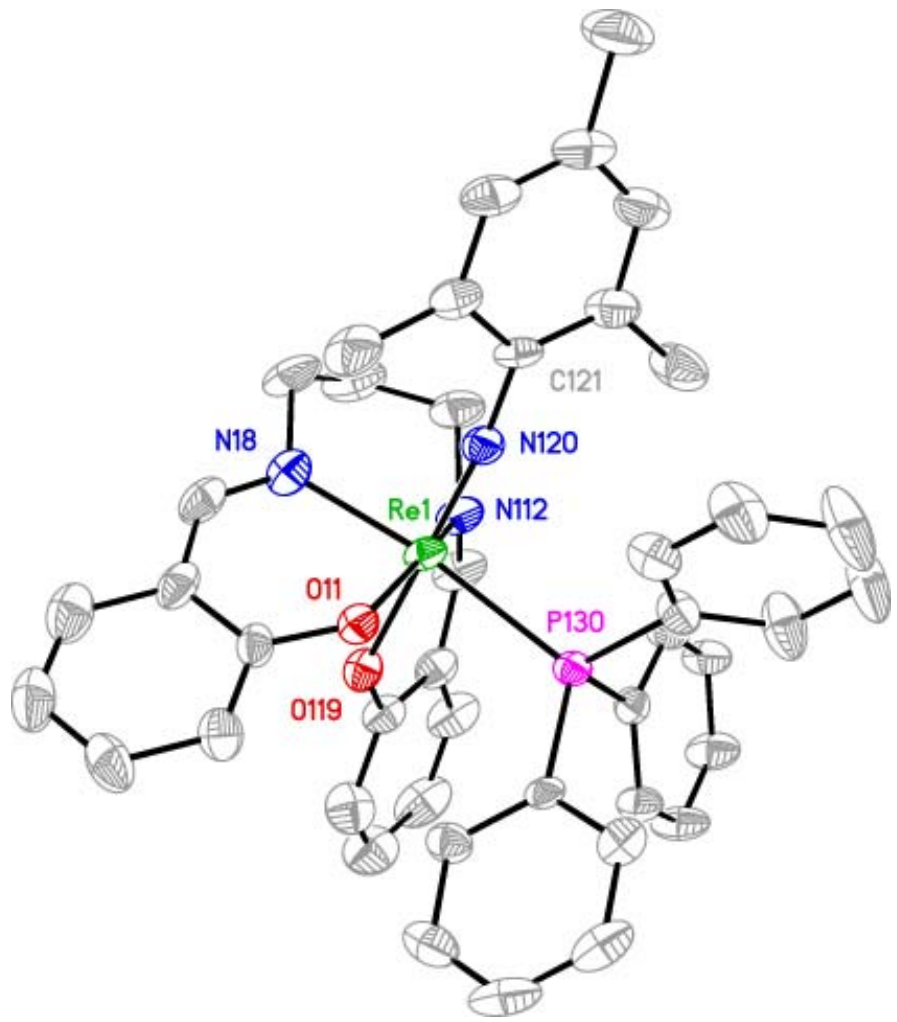

Table S5. Bond Angles in Degrees for [Re(NMes) $\left(\mathrm{PPh}_{3}\right)$ saldach $\left.^{+}\right]$, 7a

\begin{tabular}{|c|c|c|c|c|c|c|}
\hline Anole $\underline{\text { Atom } 1}$ & $\underline{\text { Atom } 2}$ & Atom 3 & Angle & Atom 1 & $\underline{\text { Atom }} \underline{2}$ & Atom 3 \\
\hline $\begin{array}{l}\mathrm{N} 120 \\
1208(4)\end{array}$ & $\operatorname{Re} 1$ & O119 & $178.86(15)$ & C19 & N18 & $\operatorname{Re} 1$ \\
\hline $\begin{array}{l}\text { N120 } \\
118.3(5)\end{array}$ & $\operatorname{Re} 1$ & O11 & $100.27(17)$ & $\mathrm{C} 27$ & $\mathrm{~N} 28$ & $\mathrm{C} 29$ \\
\hline $\begin{array}{l}\text { O119 } \\
122.2(4)\end{array}$ & Re1 & O11 & $80.87(14)$ & $\mathrm{C} 27$ & N28 & $\operatorname{Re} 2$ \\
\hline $\begin{array}{l}\text { N120 } \\
118.9(4)\end{array}$ & $\operatorname{Re} 1$ & N112 & $96.54(19)$ & $\mathrm{C} 29$ & $\mathrm{~N} 28$ & $\operatorname{Re} 2$ \\
\hline $\begin{array}{l}\text { O119 } \\
115.4(5)\end{array}$ & $\operatorname{Re} 1$ & N112 & $82.32(16)$ & C113 & N112 & C111 \\
\hline $\begin{array}{l}\text { O11 } \\
128.2(4)\end{array}$ & $\operatorname{Re} 1$ & N112 & $162.57(16)$ & $\mathrm{C} 113$ & N112 & $\operatorname{Re} 1$ \\
\hline $\begin{array}{l}\text { N120 } \\
114.3(4)\end{array}$ & $\operatorname{Re} 1$ & N18 & $91.43(19)$ & C111 & N112 & Re1 \\
\hline $\begin{array}{l}\text { O119 } \\
173.1(4)\end{array}$ & $\operatorname{Re} 1$ & N18 & $88.44(16)$ & $\mathrm{C} 121$ & N120 & Re1 \\
\hline $\begin{array}{l}\text { O11 } \\
114.6(5)\end{array}$ & $\operatorname{Re} 1$ & N18 & $89.18(15)$ & $\mathrm{C} 213$ & $\mathrm{~N} 212$ & $\mathrm{C} 211$ \\
\hline $\begin{array}{l}\text { N112 } \\
128.4(4)\end{array}$ & $\operatorname{Re} 1$ & N18 & $85.78(17)$ & $\mathrm{C} 213$ & $\mathrm{~N} 212$ & $\operatorname{Re} 2$ \\
\hline $\begin{array}{l}\text { N120 } \\
115.3(4)\end{array}$ & $\operatorname{Re} 1$ & P130 & $94.38(14)$ & $\mathrm{C} 211$ & $\mathrm{~N} 212$ & $\operatorname{Re} 2$ \\
\hline O119 & $\operatorname{Re} 1$ & P130 & $85.84(10)$ & $\mathrm{C} 221$ & N220 & Re2 \\
\hline
\end{tabular}




\begin{tabular}{|c|c|c|c|c|c|c|}
\hline $\begin{array}{l}\text { 176.2(4) } \\
\text { O11 }\end{array}$ & Re1 & P130 & $85.53(10)$ & O11 & $\mathrm{C} 11$ & $\mathrm{C} 12$ \\
\hline $117.4(5)$ & & & & & & \\
\hline $\begin{array}{l}\text { N112 } \\
124.0(5)\end{array}$ & Re1 & P130 & $97.83(13)$ & O11 & $\mathrm{C} 11$ & $\mathrm{C} 16$ \\
\hline $\begin{array}{l}\text { N18 } \\
118.6(5)\end{array}$ & Re1 & P130 & $172.75(12)$ & $\mathrm{C} 12$ & $\mathrm{C} 11$ & $\mathrm{C} 16$ \\
\hline $\begin{array}{l}\mathrm{N} 220 \\
121.2(5)\end{array}$ & $\operatorname{Re} 2$ & O219 & $179.49(18)$ & $\mathrm{C} 13$ & $\mathrm{C} 12$ & $\mathrm{C} 11$ \\
\hline $\begin{array}{l}\text { N220 } \\
119.4(5)\end{array}$ & $\operatorname{Re} 2$ & $\mathrm{O} 21$ & $99.76(17)$ & $\mathrm{C} 13$ & $\mathrm{C} 12$ & $\mathrm{H} 12$ \\
\hline $\begin{array}{l}\mathrm{O} 219 \\
119.4(5)\end{array}$ & $\operatorname{Re} 2$ & $\mathrm{O} 21$ & $80.73(14)$ & $\mathrm{C} 11$ & $\mathrm{C} 12$ & H12 \\
\hline $\begin{array}{l}\text { N220 } \\
120.7(6)\end{array}$ & $\mathrm{Re} 2$ & N212 & $97.5(2)$ & $\mathrm{C} 14$ & $\mathrm{C} 13$ & $\mathrm{C} 12$ \\
\hline $\begin{array}{l}\mathrm{O} 219 \\
119.6(6)\end{array}$ & $\mathrm{Re} 2$ & N212 & $81.99(18)$ & $\mathrm{C} 14$ & $\mathrm{C} 13$ & H13 \\
\hline $\begin{array}{l}\mathrm{O} 21 \\
119.6(6)\end{array}$ & $\mathrm{Re} 2$ & N212 & $161.95(18)$ & $\mathrm{C} 12$ & $\mathrm{C} 13$ & H13 \\
\hline $\begin{array}{l}\text { N220 } \\
118.7(6)\end{array}$ & $\operatorname{Re} 2$ & N28 & $91.2(2)$ & $\mathrm{C} 15$ & $\mathrm{C} 14$ & $\mathrm{C} 13$ \\
\hline $\begin{array}{l}\text { O219 } \\
120.6(6)\end{array}$ & $\operatorname{Re} 2$ & N28 & $88.96(17)$ & $\mathrm{C} 15$ & $\mathrm{C} 14$ & H14 \\
\hline $\begin{array}{l}\mathrm{O} 21 \\
120.6(6)\end{array}$ & $\operatorname{Re} 2$ & N28 & $88.75(16)$ & $\mathrm{C} 13$ & $\mathrm{C} 14$ & H14 \\
\hline $\begin{array}{l}\mathrm{N} 212 \\
122.3(6)\end{array}$ & $\operatorname{Re} 2$ & N28 & $85.74(19)$ & $\mathrm{C} 14$ & $\mathrm{C} 15$ & $\mathrm{C} 16$ \\
\hline $\begin{array}{l}\text { N220 } \\
118.9(6)\end{array}$ & $\operatorname{Re} 2$ & P230 & $93.74(15)$ & $\mathrm{C} 14$ & $\mathrm{C} 15$ & H15 \\
\hline $\begin{array}{l}\text { O219 } \\
118.9(6)\end{array}$ & $\operatorname{Re} 2$ & P230 & $86.15(10)$ & $\mathrm{C} 16$ & $\mathrm{C} 15$ & H15 \\
\hline $\begin{array}{l}\mathrm{O} 21 \\
118.5(5)\end{array}$ & $\operatorname{Re} 2$ & P230 & $85.41(10)$ & $\mathrm{C} 15$ & $\mathrm{C} 16$ & $\mathrm{C} 11$ \\
\hline $\begin{array}{l}\text { N212 } \\
117.3(5)\end{array}$ & $\operatorname{Re} 2$ & P230 & $98.62(14)$ & $\mathrm{C} 15$ & $\mathrm{C} 16$ & $\mathrm{C} 17$ \\
\hline $\begin{array}{l}\mathrm{N} 28 \\
124.2(5)\end{array}$ & $\operatorname{Re} 2$ & P230 & $172.92(14)$ & $\mathrm{C} 11$ & $\mathrm{C} 16$ & $\mathrm{C} 17$ \\
\hline $\begin{array}{l}\text { C131 } \\
128.6(5)\end{array}$ & P130 & C137 & $104.6(2)$ & N18 & $\mathrm{C} 17$ & C16 \\
\hline $\begin{array}{l}\text { C131 } \\
115.7(5)\end{array}$ & P130 & C143 & $104.8(2)$ & N18 & $\mathrm{C} 17$ & H17 \\
\hline $\begin{array}{l}\text { C137 } \\
115.7(5)\end{array}$ & P130 & C143 & $102.4(2)$ & $\mathrm{C} 16$ & $\mathrm{C} 17$ & H17 \\
\hline $\begin{array}{l}\text { C131 } \\
113.4(5)\end{array}$ & P130 & Re1 & 114.94(17) & N18 & C19 & C110 \\
\hline $\begin{array}{l}\text { C137 } \\
108.9(5)\end{array}$ & $\mathrm{P} 130$ & Re1 & $113.60(19)$ & N18 & C19 & H19A \\
\hline $\begin{array}{l}\text { C143 } \\
108.9(5)\end{array}$ & P130 & Re1 & $115.15(16)$ & C110 & $\mathrm{C} 19$ & H19A \\
\hline $\begin{array}{l}\text { C231 } \\
108.9(5)\end{array}$ & $\mathrm{P} 230$ & $\mathrm{C} 243$ & $102.3(2)$ & N18 & C19 & H19B \\
\hline $\begin{array}{l}\text { C231 } \\
108.9(5)\end{array}$ & $\mathrm{P} 230$ & C237 & $104.9(2)$ & C110 & C19 & H19B \\
\hline $\begin{array}{l}\text { C243 } \\
107.7(5)\end{array}$ & P230 & $\mathrm{C} 237$ & $104.5(2)$ & H19A & C19 & H19B \\
\hline $\mathrm{C} 231$ & P230 & $\operatorname{Re} 2$ & $112.1(2)$ & $\mathrm{O} 21$ & $\mathrm{C} 21$ & $\mathrm{C} 22$ \\
\hline
\end{tabular}




\begin{tabular}{|c|c|c|c|c|c|c|}
\hline $\begin{array}{l}117.1(5) \\
\text { C243 }\end{array}$ & P230 & $\operatorname{Re} 2$ & $115.44(16)$ & $\mathrm{O} 21$ & $\mathrm{C} 21$ & $\mathrm{C} 26$ \\
\hline $\begin{array}{l}124.0(5) \\
\text { C237 }\end{array}$ & P230 & $\operatorname{Re} 2$ & $116.16(17)$ & $\mathrm{C} 22$ & $\mathrm{C} 21$ & $\mathrm{C} 26$ \\
\hline $\begin{array}{l}118.9(5) \\
\text { C11 }\end{array}$ & O11 & Re1 & $123.9(3)$ & $\mathrm{C} 21$ & $\mathrm{C} 22$ & $\mathrm{C} 23$ \\
\hline $\begin{array}{l}121.2(6) \\
\mathrm{C} 21 \\
119.4(6)\end{array}$ & $\mathrm{O} 21$ & $\operatorname{Re} 2$ & $124.3(3)$ & $\mathrm{C} 21$ & $\mathrm{C} 22$ & $\mathrm{H} 22$ \\
\hline $\begin{array}{l}\text { C119 } \\
119.4(6)\end{array}$ & O119 & Re1 & $133.4(3)$ & $\mathrm{C} 23$ & $\mathrm{C} 22$ & $\mathrm{H} 22$ \\
\hline $\begin{array}{l}\text { C219 } \\
120.4(6)\end{array}$ & $\mathrm{O} 219$ & $\operatorname{Re} 2$ & $132.3(3)$ & $\mathrm{C} 24$ & $\mathrm{C} 23$ & $\mathrm{C} 22$ \\
\hline $\begin{array}{l}\text { C17 } \\
119.8(6)\end{array}$ & N18 & C19 & $116.8(5)$ & $\mathrm{C} 24$ & $\mathrm{C} 23$ & $\mathrm{H} 23$ \\
\hline $\begin{array}{l}\text { C17 } \\
119.8(6)\end{array}$ & N18 & Re1 & $121.9(4)$ & $\mathrm{C} 22$ & $\mathrm{C} 23$ & $\mathrm{H} 23$ \\
\hline $\begin{array}{l}\mathrm{C} 23 \\
119.9(5)\end{array}$ & $\mathrm{C} 24$ & $\mathrm{C} 25$ & $119.5(6)$ & O119 & C119 & $\mathrm{C} 118$ \\
\hline $\begin{array}{l}\mathrm{C} 23 \\
121.8(5)\end{array}$ & $\mathrm{C} 24$ & $\mathrm{H} 24$ & $120.2(6)$ & O119 & C119 & $\mathrm{C} 114$ \\
\hline $\begin{array}{l}\mathrm{C} 25 \\
118.3(5)\end{array}$ & $\mathrm{C} 24$ & $\mathrm{H} 24$ & $120.2(6)$ & C118 & C119 & $\mathrm{C} 114$ \\
\hline $\begin{array}{l}\mathrm{C} 24 \\
121.1(5)\end{array}$ & $\mathrm{C} 25$ & $\mathrm{C} 26$ & $121.2(6)$ & N120 & C121 & $\mathrm{C} 122$ \\
\hline $\begin{array}{l}\mathrm{C} 24 \\
118.0(5)\end{array}$ & $\mathrm{C} 25$ & $\mathrm{H} 25$ & $119.4(6)$ & N120 & $\mathrm{C} 121$ & $\mathrm{C} 126$ \\
\hline $\begin{array}{l}\mathrm{C} 26 \\
120.8(5)\end{array}$ & $\mathrm{C} 25$ & $\mathrm{H} 25$ & $119.4(6)$ & $\mathrm{C} 122$ & $\mathrm{C} 121$ & $\mathrm{C} 126$ \\
\hline $\begin{array}{l}\mathrm{C} 25 \\
117.9(6)\end{array}$ & $\mathrm{C} 26$ & $\mathrm{C} 21$ & $118.6(6)$ & $\mathrm{C} 123$ & $\mathrm{C} 122$ & $\mathrm{C} 121$ \\
\hline $\begin{array}{l}\mathrm{C} 25 \\
120.7(5)\end{array}$ & $\mathrm{C} 26$ & $\mathrm{C} 27$ & $116.8(5)$ & $\mathrm{C} 123$ & $\mathrm{C} 122$ & $\mathrm{C} 127$ \\
\hline $\begin{array}{l}\mathrm{C} 21 \\
121.4(5)\end{array}$ & $\mathrm{C} 26$ & $\mathrm{C} 27$ & $124.5(5)$ & C121 & C122 & $\mathrm{C} 127$ \\
\hline $\begin{array}{l}\mathrm{N} 28 \\
122.5(6)\end{array}$ & $\mathrm{C} 27$ & $\mathrm{C} 26$ & $128.3(5)$ & C124 & $\mathrm{C} 123$ & $\mathrm{C} 122$ \\
\hline $\begin{array}{l}\text { N28 } \\
118.8(6)\end{array}$ & $\mathrm{C} 27$ & $\mathrm{H} 27$ & $115.8(5)$ & $\mathrm{C} 124$ & $\mathrm{C} 123$ & H123 \\
\hline $\begin{array}{l}\mathrm{C} 26 \\
118.8(6)\end{array}$ & $\mathrm{C} 27$ & $\mathrm{H} 27$ & $115.8(5)$ & C122 & $\mathrm{C} 123$ & $\mathrm{H} 123$ \\
\hline $\begin{array}{l}\text { N28 } \\
119.0(6)\end{array}$ & C29 & C210 & $115.2(5)$ & C123 & C124 & $\mathrm{C} 125$ \\
\hline $\begin{array}{l}\text { N28 } \\
121.8(6)\end{array}$ & C29 & $\mathrm{H} 29 \mathrm{~A}$ & $108.5(5)$ & C123 & C124 & $\mathrm{C} 128$ \\
\hline $\begin{array}{l}\mathrm{C} 210 \\
119.3(6)\end{array}$ & $\mathrm{C} 29$ & $\mathrm{H} 29 \mathrm{~A}$ & $108.5(5)$ & $\mathrm{C} 125$ & $\mathrm{C} 124$ & $\mathrm{C} 128$ \\
\hline $\begin{array}{l}\text { N28 } \\
122.6(6)\end{array}$ & $\mathrm{C} 29$ & H29B & $108.5(5)$ & C126 & $\mathrm{C} 125$ & $\mathrm{C} 124$ \\
\hline $\begin{array}{l}\text { C210 } \\
118.7(6)\end{array}$ & $\mathrm{C} 29$ & $\mathrm{H} 29 \mathrm{~B}$ & $108.5(5)$ & C126 & C125 & H125 \\
\hline $\begin{array}{l}\mathrm{H} 29 \mathrm{~A} \\
118.7(6)\end{array}$ & $\mathrm{C} 29$ & H29B & $107.5(5)$ & C124 & C125 & H125 \\
\hline $\begin{array}{l}\text { C111 } \\
117.1(5)\end{array}$ & C110 & C19 & $115.2(5)$ & C125 & C126 & $\mathrm{C} 121$ \\
\hline C111 & C110 & H11A & $108.5(5)$ & C125 & C126 & C129 \\
\hline
\end{tabular}




\begin{tabular}{|c|c|c|c|c|c|c|}
\hline $\begin{array}{l}122.2(5) \\
\text { C19 }\end{array}$ & $\mathrm{C} 110$ & H11A & $108.5(5)$ & C121 & C126 & C129 \\
\hline $\begin{array}{l}\text { 120.7(5) } \\
\text { C111 }\end{array}$ & $\mathrm{C} 110$ & H11B & $108.5(5)$ & $\mathrm{C} 122$ & C127 & $\mathrm{H} 12 \mathrm{~A}$ \\
\hline $\begin{array}{l}109.5(5) \\
\text { C19 } \\
109.5(5)\end{array}$ & $\mathrm{C} 110$ & H11B & $108.5(5)$ & $\mathrm{C} 122$ & C127 & H12B \\
\hline $\begin{array}{l}\text { H11A } \\
109.5(5)\end{array}$ & $\mathrm{C} 110$ & H11B & $107.5(5)$ & $\mathrm{H} 12 \mathrm{~A}$ & C127 & H12B \\
\hline $\begin{array}{l}\text { C110 } \\
109.5(5)\end{array}$ & C111 & N112 & $107.5(5)$ & C122 & C127 & $\mathrm{H} 12 \mathrm{C}$ \\
\hline $\begin{array}{l}\text { C110 } \\
109.5(5)\end{array}$ & C111 & $\mathrm{H} 11 \mathrm{C}$ & $110.2(5)$ & $\mathrm{H} 12 \mathrm{~A}$ & C127 & $\mathrm{H} 12 \mathrm{C}$ \\
\hline $\begin{array}{l}\text { N112 } \\
109.5(5)\end{array}$ & C111 & $\mathrm{H} 11 \mathrm{C}$ & $110.2(5)$ & H12B & C127 & $\mathrm{H} 12 \mathrm{C}$ \\
\hline $\begin{array}{l}C 110 \\
109.5(5)\end{array}$ & C111 & H11D & $110.2(5)$ & $\mathrm{C} 124$ & C128 & H12D \\
\hline $\begin{array}{l}\text { N112 } \\
109.5(5)\end{array}$ & C111 & H11D & $110.2(5)$ & C124 & C128 & $\mathrm{H} 12 \mathrm{E}$ \\
\hline $\begin{array}{l}\text { H11C } \\
109.5(5)\end{array}$ & C111 & H11D & $108.5(5)$ & H12D & C128 & $\mathrm{H} 12 \mathrm{E}$ \\
\hline $\begin{array}{l}\text { N112 } \\
109.5(5)\end{array}$ & C113 & C114 & $126.6(5)$ & $\mathrm{C} 124$ & C128 & $\mathrm{H} 12 \mathrm{~F}$ \\
\hline $\begin{array}{l}\text { N112 } \\
109.5(5)\end{array}$ & C113 & H113 & $116.7(5)$ & H12D & C128 & $\mathrm{H} 12 \mathrm{~F}$ \\
\hline $\begin{array}{l}\text { C114 } \\
109.5(5)\end{array}$ & C113 & H113 & $116.7(5)$ & H12E & C128 & $\mathrm{H} 12 \mathrm{~F}$ \\
\hline $\begin{array}{l}\text { C119 } \\
109.5(5)\end{array}$ & C114 & C115 & $120.0(5)$ & $\mathrm{C} 126$ & C129 & $\mathrm{H} 12 \mathrm{G}$ \\
\hline $\begin{array}{l}C 119 \\
109.5(5)\end{array}$ & $\mathrm{C} 114$ & $\mathrm{C} 113$ & $120.1(5)$ & C126 & C129 & $\mathrm{H} 12 \mathrm{H}$ \\
\hline $\begin{array}{l}\text { C115 } \\
109.5(5)\end{array}$ & $\mathrm{C} 114$ & C113 & $119.4(5)$ & $\mathrm{H} 12 \mathrm{G}$ & C129 & $\mathrm{H} 12 \mathrm{H}$ \\
\hline $\begin{array}{l}\text { C116 } \\
109.5(5)\end{array}$ & C115 & C114 & $120.6(6)$ & C126 & C129 & H12I \\
\hline $\begin{array}{l}\text { C116 } \\
109.5(5)\end{array}$ & C115 & H115 & 119.7(6) & H12G & C129 & H12I \\
\hline $\begin{array}{l}\text { C114 } \\
109.5(5)\end{array}$ & C115 & H115 & $119.7(6)$ & $\mathrm{H} 12 \mathrm{H}$ & C129 & H12I \\
\hline $\begin{array}{l}\text { C115 } \\
118.6(5)\end{array}$ & C116 & C117 & $120.2(6)$ & C132 & C131 & C136 \\
\hline $\begin{array}{l}\text { C115 } \\
122.8(4)\end{array}$ & C116 & H116 & $119.9(6)$ & C132 & C131 & P130 \\
\hline $\begin{array}{l}\text { C117 } \\
118.6(4)\end{array}$ & C116 & H116 & 119.9(6) & C136 & C131 & P130 \\
\hline $\begin{array}{l}\text { C118 } \\
120.4(5)\end{array}$ & C117 & C116 & $120.2(6)$ & $\mathrm{C} 133$ & $\mathrm{C} 132$ & C131 \\
\hline $\begin{array}{l}\text { C118 } \\
119.8(5)\end{array}$ & C117 & H117 & $119.9(6)$ & C133 & C132 & H132 \\
\hline $\begin{array}{l}\text { C116 } \\
119.8(5)\end{array}$ & C117 & H117 & 119.9(6) & C131 & C132 & H132 \\
\hline $\begin{array}{l}\text { C117 } \\
120.1(5)\end{array}$ & C118 & C119 & $120.7(6)$ & C134 & C133 & C132 \\
\hline $\begin{array}{l}\text { C117 } \\
120.0(5)\end{array}$ & C118 & H118 & $119.6(6)$ & C134 & C133 & H133 \\
\hline C119 & C118 & H118 & $119.6(6)$ & C132 & C133 & H133 \\
\hline
\end{tabular}




\begin{tabular}{|c|c|c|c|c|c|c|}
\hline $\begin{array}{l}120.0(5) \\
\text { C133 }\end{array}$ & $\mathrm{C} 134$ & C135 & $120.5(6)$ & $\mathrm{C} 211$ & $\mathrm{C} 210$ & $\mathrm{H} 21 \mathrm{~B}$ \\
\hline $\begin{array}{l}108.8(6) \\
\text { C133 }\end{array}$ & $\mathrm{C} 134$ & H134 & $119.8(6)$ & $\mathrm{C} 29$ & $\mathrm{C} 210$ & $\mathrm{H} 21 \mathrm{~B}$ \\
\hline $\begin{array}{l}108.8(6) \\
C 135 \\
107.7(6)\end{array}$ & $\mathrm{C} 134$ & H134 & $119.8(6)$ & $\mathrm{H} 21 \mathrm{~A}$ & $\mathrm{C} 210$ & $\mathrm{H} 21 \mathrm{~B}$ \\
\hline $\begin{array}{l}\text { C136 } \\
108.6(6)\end{array}$ & C135 & C134 & $120.0(5)$ & N212 & $\mathrm{C} 211$ & $\mathrm{C} 210$ \\
\hline $\begin{array}{l}\text { C136 } \\
110.0(6)\end{array}$ & C135 & H135 & $120.0(5)$ & N212 & $\mathrm{C} 211$ & $\mathrm{H} 21 \mathrm{C}$ \\
\hline $\begin{array}{l}\text { C134 } \\
110.0(6)\end{array}$ & C135 & H135 & $120.0(5)$ & $\mathrm{C} 210$ & $\mathrm{C} 211$ & $\mathrm{H} 21 \mathrm{C}$ \\
\hline $\begin{array}{l}\text { C135 } \\
110.0(6)\end{array}$ & C136 & C131 & $120.4(5)$ & $\mathrm{N} 212$ & $\mathrm{C} 211$ & $\mathrm{H} 21 \mathrm{D}$ \\
\hline $\begin{array}{l}\text { C135 } \\
110.0(6)\end{array}$ & C136 & H136 & $119.8(5)$ & $\mathrm{C} 210$ & $\mathrm{C} 211$ & $\mathrm{H} 21 \mathrm{D}$ \\
\hline $\begin{array}{l}\text { C131 } \\
108.3(6)\end{array}$ & C136 & H136 & $119.8(5)$ & $\mathrm{H} 21 \mathrm{C}$ & $\mathrm{C} 211$ & $\mathrm{H} 21 \mathrm{D}$ \\
\hline $\begin{array}{l}\mathrm{C} 142 \\
127.1(5)\end{array}$ & C137 & C138 & 119.2(5) & $\mathrm{N} 212$ & $\mathrm{C} 213$ & $\mathrm{C} 214$ \\
\hline $\begin{array}{l}\text { C142 } \\
116.5(5)\end{array}$ & C137 & P130 & 118.2(4) & $\mathrm{N} 212$ & $\mathrm{C} 213$ & $\mathrm{H} 213$ \\
\hline $\begin{array}{l}\text { C138 } \\
116.5(5)\end{array}$ & C137 & P130 & $122.5(4)$ & $\mathrm{C} 214$ & $\mathrm{C} 213$ & $\mathrm{H} 213$ \\
\hline $\begin{array}{l}\text { C139 } \\
118.8(6)\end{array}$ & C138 & C137 & $119.5(6)$ & C215 & $\mathrm{C} 214$ & C219 \\
\hline $\begin{array}{l}\text { C139 } \\
121.0(6)\end{array}$ & $\mathrm{C} 138$ & H138 & $120.2(6)$ & $\mathrm{C} 215$ & $\mathrm{C} 214$ & $\mathrm{C} 213$ \\
\hline $\begin{array}{l}\text { C137 } \\
119.8(5)\end{array}$ & C138 & H138 & $120.2(6)$ & C219 & $\mathrm{C} 214$ & C213 \\
\hline $\begin{array}{l}\text { C140 } \\
121.3(7)\end{array}$ & C139 & C138 & $120.5(6)$ & $\mathrm{C} 216$ & $\mathrm{C} 215$ & $\mathrm{C} 214$ \\
\hline $\begin{array}{l}\text { C140 } \\
119.4(7)\end{array}$ & C139 & H139 & 119.7(6) & C216 & $\mathrm{C} 215$ & H215 \\
\hline $\begin{array}{l}\text { C138 } \\
119.4(7)\end{array}$ & C139 & H139 & $119.7(6)$ & $\mathrm{C} 214$ & $\mathrm{C} 215$ & $\mathrm{H} 215$ \\
\hline $\begin{array}{l}\text { C139 } \\
120.3(7)\end{array}$ & C140 & C141 & $120.9(6)$ & $\mathrm{C} 218$ & $\mathrm{C} 217$ & $\mathrm{C} 216$ \\
\hline $\begin{array}{l}\text { C139 } \\
119.8(7)\end{array}$ & $\mathrm{C} 140$ & $\mathrm{H} 140$ & $119.6(6)$ & $\mathrm{C} 218$ & $\mathrm{C} 217$ & $\mathrm{H} 217$ \\
\hline $\begin{array}{l}\text { C141 } \\
119.8(7)\end{array}$ & C140 & H140 & $119.6(6)$ & C216 & $\mathrm{C} 217$ & $\mathrm{H} 217$ \\
\hline $\begin{array}{l}\text { C142 } \\
119.9(6)\end{array}$ & C141 & C140 & 119.3(6) & $\mathrm{C} 217$ & $\mathrm{C} 218$ & C219 \\
\hline $\begin{array}{l}\text { C142 } \\
120.0(6)\end{array}$ & C141 & H141 & $120.4(6)$ & $\mathrm{C} 217$ & $\mathrm{C} 218$ & $\mathrm{H} 218$ \\
\hline $\begin{array}{l}\text { C140 } \\
120.0(6)\end{array}$ & C141 & H141 & $120.4(6)$ & $\mathrm{C} 219$ & $\mathrm{C} 218$ & $\mathrm{H} 218$ \\
\hline $\begin{array}{l}\text { C141 } \\
118.4(5)\end{array}$ & C142 & C137 & $120.6(6)$ & $\mathrm{O} 219$ & $\mathrm{C} 219$ & $\mathrm{C} 218$ \\
\hline $\begin{array}{l}\text { C141 } \\
121.9(5)\end{array}$ & C142 & H142 & $119.7(6)$ & $\mathrm{O} 219$ & $\mathrm{C} 219$ & $\mathrm{C} 214$ \\
\hline $\begin{array}{l}\text { C137 } \\
119.6(6)\end{array}$ & C142 & H142 & $119.7(6)$ & $\mathrm{C} 218$ & $\mathrm{C} 219$ & $\mathrm{C} 214$ \\
\hline $\mathrm{C} 144$ & C143 & C148 & $119.6(5)$ & N220 & $\mathrm{C} 221$ & $\mathrm{C} 226$ \\
\hline
\end{tabular}




\begin{tabular}{|c|c|c|c|c|c|c|}
\hline $\begin{array}{l}121.6(5) \\
\text { C144 }\end{array}$ & C143 & P130 & $120.1(4)$ & N220 & $\mathrm{C} 221$ & $\mathrm{C} 222$ \\
\hline $\begin{array}{l}118.3(5) \\
\text { C148 }\end{array}$ & C143 & P130 & $120.3(4)$ & C226 & $\mathrm{C} 221$ & $\mathrm{C} 222$ \\
\hline $\begin{array}{l}120.1(5) \\
\mathrm{C} 143\end{array}$ & C144 & $\mathrm{C} 145$ & $119.6(6)$ & C223 & $\mathrm{C} 222$ & $\mathrm{C} 221$ \\
\hline $118.4(5)$ & & & & & & \\
\hline $\begin{array}{l}\text { C143 } \\
122.1(5)\end{array}$ & C144 & $\mathrm{H} 144$ & $120.2(6)$ & C223 & $\mathrm{C} 222$ & $\mathrm{C} 227$ \\
\hline $\begin{array}{l}\mathrm{C} 145 \\
119.5(5)\end{array}$ & C144 & $\mathrm{H} 144$ & $120.2(6)$ & $\mathrm{C} 221$ & $\mathrm{C} 222$ & $\mathrm{C} 227$ \\
\hline $\begin{array}{l}\mathrm{C} 146 \\
122.9(6)\end{array}$ & C145 & C144 & $120.5(6)$ & C222 & $\mathrm{C} 223$ & $\mathrm{C} 224$ \\
\hline $\begin{array}{l}\mathrm{C} 146 \\
118.5(6)\end{array}$ & C145 & $\mathrm{H} 145$ & $119.8(6)$ & C222 & $\mathrm{C} 223$ & $\mathrm{H} 223$ \\
\hline $\begin{array}{l}\mathrm{C} 144 \\
118.5(6)\end{array}$ & C145 & H145 & $119.8(6)$ & $\mathrm{C} 224$ & $\mathrm{C} 223$ & $\mathrm{H} 223$ \\
\hline $\begin{array}{l}\mathrm{C} 147 \\
118.7(6)\end{array}$ & C146 & C145 & $119.9(6)$ & $\mathrm{C} 225$ & $\mathrm{C} 224$ & $\mathrm{C} 223$ \\
\hline $\begin{array}{l}\mathrm{C} 147 \\
119.8(6)\end{array}$ & C146 & H146 & $120.1(6)$ & $\mathrm{C} 225$ & $\mathrm{C} 224$ & C228 \\
\hline $\begin{array}{l}\mathrm{C} 145 \\
121.4(6)\end{array}$ & C146 & H146 & $120.1(6)$ & C223 & C224 & C228 \\
\hline $\begin{array}{l}\mathrm{C} 146 \\
121.5(5)\end{array}$ & C147 & C148 & $121.3(6)$ & $\mathrm{C} 224$ & $\mathrm{C} 225$ & $\mathrm{C} 226$ \\
\hline $\begin{array}{l}\mathrm{C} 146 \\
119.3(5)\end{array}$ & C147 & H147 & $119.4(6)$ & C224 & $\mathrm{C} 225$ & $\mathrm{H} 225$ \\
\hline $\begin{array}{l}\mathrm{C} 148 \\
119.3(5)\end{array}$ & C147 & H147 & $119.4(6)$ & C226 & $\mathrm{C} 225$ & $\mathrm{H} 225$ \\
\hline $\begin{array}{l}\text { C147 } \\
118.3(6)\end{array}$ & C148 & C143 & $119.0(5)$ & $\mathrm{C} 225$ & $\mathrm{C} 226$ & $\mathrm{C} 221$ \\
\hline $\begin{array}{l}\mathrm{C} 147 \\
119.9(5)\end{array}$ & C148 & H148 & $120.5(5)$ & $\mathrm{C} 225$ & $\mathrm{C} 226$ & C229 \\
\hline $\begin{array}{l}\mathrm{C} 143 \\
121.7(5)\end{array}$ & C148 & H148 & $120.5(5)$ & C221 & C226 & C229 \\
\hline $\begin{array}{l}\mathrm{C} 211 \\
109.5(5)\end{array}$ & $\mathrm{C} 210$ & C29 & $113.8(6)$ & C222 & $\mathrm{C} 227$ & $\mathrm{H} 22 \mathrm{~A}$ \\
\hline $\begin{array}{l}\text { C211 } \\
109.5(5)\end{array}$ & $\mathrm{C} 210$ & $\mathrm{H} 21 \mathrm{~A}$ & $108.8(6)$ & C222 & $\mathrm{C} 227$ & $\mathrm{H} 22 \mathrm{~B}$ \\
\hline $\begin{array}{l}\mathrm{C} 29 \\
109.5(5)\end{array}$ & $\mathrm{C} 210$ & $\mathrm{H} 21 \mathrm{~A}$ & $108.8(6)$ & $\mathrm{H} 22 \mathrm{~A}$ & $\mathrm{C} 227$ & $\mathrm{H} 22 \mathrm{~B}$ \\
\hline $\begin{array}{l}\mathrm{C} 222 \\
121.1(5)\end{array}$ & $\mathrm{C} 227$ & $\mathrm{H} 22 \mathrm{C}$ & $109.5(5)$ & C241 & C242 & C237 \\
\hline $\begin{array}{l}\mathrm{H} 22 \mathrm{~A} \\
119.5(5)\end{array}$ & $\mathrm{C} 227$ & $\mathrm{H} 22 \mathrm{C}$ & $109.5(5)$ & C241 & C242 & $\mathrm{H} 242$ \\
\hline $\begin{array}{l}\text { H22B } \\
119.5(5)\end{array}$ & $\mathrm{C} 227$ & $\mathrm{H} 22 \mathrm{C}$ & $109.5(5)$ & C237 & $\mathrm{C} 242$ & $\mathrm{H} 242$ \\
\hline $\begin{array}{l}\mathrm{C} 224 \\
119.3(5)\end{array}$ & $\mathrm{C} 228$ & H22D & $109.5(5)$ & C244 & $\mathrm{C} 243$ & C248 \\
\hline $\begin{array}{l}\mathrm{C} 224 \\
120.3(4)\end{array}$ & $\mathrm{C} 228$ & $\mathrm{H} 22 \mathrm{E}$ & $109.5(5)$ & C244 & C243 & P230 \\
\hline $\begin{array}{l}\mathrm{H} 22 \mathrm{D} \\
120.3(4)\end{array}$ & $\mathrm{C} 228$ & $\mathrm{H} 22 \mathrm{E}$ & $109.5(5)$ & C248 & C243 & P230 \\
\hline $\begin{array}{l}\mathrm{C} 224 \\
121.2(5)\end{array}$ & $\mathrm{C} 228$ & $\mathrm{H} 22 \mathrm{~F}$ & $109.5(5)$ & C243 & C244 & C245 \\
\hline $\mathrm{H} 22 \mathrm{D}$ & $\mathrm{C} 228$ & $\mathrm{H} 22 \mathrm{~F}$ & $109.5(5)$ & C243 & C244 & $\mathrm{H} 244$ \\
\hline
\end{tabular}




\begin{tabular}{|c|c|c|c|c|c|c|}
\hline $\begin{array}{l}119.4(5) \\
\text { H22E }\end{array}$ & C228 & $\mathrm{H} 22 \mathrm{~F}$ & $109.5(5)$ & C245 & C244 & $\mathrm{H} 244$ \\
\hline $\begin{array}{l}119.4(5) \\
\text { C226 }\end{array}$ & C229 & $\mathrm{H} 22 \mathrm{G}$ & $109.5(5)$ & C246 & $\mathrm{C} 245$ & $\mathrm{C} 244$ \\
\hline $\begin{array}{l}119.5(6) \\
\text { C226 }\end{array}$ & $\mathrm{C} 229$ & $\mathrm{H} 22 \mathrm{H}$ & $109.5(5)$ & C246 & $\mathrm{C} 245$ & $\mathrm{H} 245$ \\
\hline $\begin{array}{l}120.2(6) \\
\mathrm{H} 22 \mathrm{G}\end{array}$ & C229 & $\mathrm{H} 22 \mathrm{H}$ & $109.5(5)$ & $\mathrm{C} 244$ & $\mathrm{C} 245$ & $\mathrm{H} 245$ \\
\hline $\begin{array}{l}120.2(6) \\
C 226 \\
120.4(6)\end{array}$ & C229 & $\mathrm{H} 22 \mathrm{I}$ & $109.5(5)$ & $\mathrm{C} 245$ & $\mathrm{C} 246$ & $\mathrm{C} 247$ \\
\hline $\begin{array}{l}\mathrm{H} 22 \mathrm{G} \\
119.8(6)\end{array}$ & $\mathrm{C} 229$ & H22I & $109.5(5)$ & $\mathrm{C} 245$ & $\mathrm{C} 246$ & $\mathrm{H} 246$ \\
\hline $\begin{array}{l}\mathrm{H} 22 \mathrm{H} \\
119.8(6)\end{array}$ & $\mathrm{C} 229$ & H22I & $109.5(5)$ & C247 & $\mathrm{C} 246$ & $\mathrm{H} 246$ \\
\hline $\begin{array}{l}\mathrm{C} 232 \\
120.2(6)\end{array}$ & $\mathrm{C} 231$ & $\mathrm{C} 236$ & $118.7(5)$ & C248 & $\mathrm{C} 247$ & $\mathrm{C} 246$ \\
\hline $\begin{array}{l}\mathrm{C} 232 \\
119.9(6)\end{array}$ & $\mathrm{C} 231$ & P230 & $118.2(4)$ & $\mathrm{C} 248$ & $\mathrm{C} 247$ & $\mathrm{H} 247$ \\
\hline $\begin{array}{l}\mathrm{C} 236 \\
119.9(6)\end{array}$ & $\mathrm{C} 231$ & P230 & $122.9(5)$ & C246 & $\mathrm{C} 247$ & $\mathrm{H} 247$ \\
\hline $\begin{array}{l}\mathrm{C} 233 \\
119.4(5)\end{array}$ & $\mathrm{C} 232$ & $\mathrm{C} 231$ & $121.1(5)$ & C247 & $\mathrm{C} 248$ & $\mathrm{C} 243$ \\
\hline $\begin{array}{l}\mathrm{C} 233 \\
120.3(5)\end{array}$ & $\mathrm{C} 232$ & H232 & $119.5(5)$ & C247 & $\mathrm{C} 248$ & $\mathrm{H} 248$ \\
\hline $\begin{array}{l}\mathrm{C} 231 \\
120.3(5)\end{array}$ & $\mathrm{C} 232$ & H232 & $119.5(5)$ & $\mathrm{C} 243$ & $\mathrm{C} 248$ & $\mathrm{H} 248$ \\
\hline $\begin{array}{l}\mathrm{C} 232 \\
120.0(7)\end{array}$ & $\mathrm{C} 233$ & C234 & $120.4(6)$ & $\mathrm{C} 215$ & $\mathrm{C} 216$ & $\mathrm{C} 217$ \\
\hline $\begin{array}{l}\text { C232 } \\
120.0(7)\end{array}$ & $\mathrm{C} 233$ & H233 & $119.8(6)$ & $\mathrm{C} 215$ & $\mathrm{C} 216$ & $\mathrm{H} 216$ \\
\hline $\begin{array}{l}\mathrm{C} 234 \\
120.0(7)\end{array}$ & $\mathrm{C} 233$ & H233 & $119.8(6)$ & $\mathrm{C} 217$ & $\mathrm{C} 216$ & H216 \\
\hline $\begin{array}{l}\mathrm{C} 235 \\
111.8(5)\end{array}$ & $\mathrm{C} 234$ & $\mathrm{C} 233$ & $119.9(6)$ & $\mathrm{C} 316$ & $\mathrm{C} 311$ & C 312 \\
\hline $\begin{array}{l}\mathrm{C} 235 \\
127.2(5)\end{array}$ & C234 & H234 & $120.1(6)$ & C316 & C311 & B3 \\
\hline $\begin{array}{l}\text { C233 } \\
120.5(5)\end{array}$ & $\mathrm{C} 234$ & H234 & $120.1(6)$ & $\mathrm{C} 312$ & $\mathrm{C} 311$ & B3 \\
\hline $\begin{array}{l}\mathrm{C} 234 \\
116.9(5)\end{array}$ & $\mathrm{C} 235$ & C236 & $120.3(7)$ & F312 & C 312 & C313 \\
\hline $\begin{array}{l}\mathrm{C} 234 \\
118.0(5)\end{array}$ & C235 & $\mathrm{H} 235$ & $119.9(7)$ & F312 & C312 & C311 \\
\hline $\begin{array}{l}\text { C236 } \\
125.1(6)\end{array}$ & $\mathrm{C} 235$ & $\mathrm{H} 235$ & $119.9(7)$ & C313 & C312 & C311 \\
\hline $\begin{array}{l}\text { C235 } \\
119.7(6)\end{array}$ & $\mathrm{C} 236$ & $\mathrm{C} 231$ & 119.6(6) & F313 & $\mathrm{C} 313$ & C314 \\
\hline $\begin{array}{l}\mathrm{C} 235 \\
120.5(6)\end{array}$ & $\mathrm{C} 236$ & H236 & $120.2(6)$ & F313 & C313 & C 312 \\
\hline $\begin{array}{l}\mathrm{C} 231 \\
119.8(6)\end{array}$ & $\mathrm{C} 236$ & H236 & $120.2(6)$ & C314 & C313 & C 312 \\
\hline $\begin{array}{l}\mathrm{C} 238 \\
120.7(6)\end{array}$ & C237 & C242 & $119.2(5)$ & F314 & C314 & C313 \\
\hline $\begin{array}{l}\text { C238 } \\
120.4(6)\end{array}$ & $\mathrm{C} 237$ & $\mathrm{P} 230$ & $121.9(4)$ & F314 & C314 & C315 \\
\hline $\mathrm{C} 242$ & C237 & P230 & $118.9(4)$ & C313 & C314 & C 315 \\
\hline
\end{tabular}




\begin{tabular}{|c|c|c|c|c|c|c|}
\hline $\begin{array}{l}118.9(6) \\
C 237 \\
120.0(6)\end{array}$ & $\mathrm{C} 238$ & C239 & $119.5(5)$ & F315 & C315 & C 314 \\
\hline $\begin{array}{l}\text { C237 } \\
120.7(6)\end{array}$ & $\mathrm{C} 238$ & $\mathrm{H} 238$ & $120.3(5)$ & F315 & C315 & C316 \\
\hline $\begin{array}{l}\text { C239 } \\
119.3(6)\end{array}$ & C238 & $\mathrm{H} 238$ & $120.3(5)$ & C314 & C315 & C316 \\
\hline $\begin{array}{l}C 240 \\
114.5(5)\end{array}$ & C239 & $\mathrm{C} 238$ & 121.1(6) & F316 & C316 & C 315 \\
\hline $\begin{array}{l}\text { C240 } \\
120.3(5)\end{array}$ & C239 & $\mathrm{H} 239$ & $119.5(6)$ & F316 & C316 & C311 \\
\hline $\begin{array}{l}\text { C238 } \\
125.1(6)\end{array}$ & C239 & $\mathrm{H} 239$ & $119.5(6)$ & C315 & $\mathrm{C} 316$ & C311 \\
\hline $\begin{array}{l}\text { C239 } \\
112.0(5)\end{array}$ & $\mathrm{C} 240$ & $\mathrm{C} 241$ & $120.0(6)$ & C322 & C321 & C326 \\
\hline $\begin{array}{l}\text { C239 } \\
129.6(6)\end{array}$ & C240 & $\mathrm{H} 240$ & $120.0(6)$ & C322 & C321 & B3 \\
\hline $\begin{array}{l}\text { C241 } \\
118.1(5)\end{array}$ & $\mathrm{C} 240$ & $\mathrm{H} 240$ & $120.0(6)$ & C326 & C321 & B3 \\
\hline $\begin{array}{l}\mathrm{C} 242 \\
116.0(6)\end{array}$ & C241 & $\mathrm{C} 240$ & 119.1(5) & F322 & C322 & C323 \\
\hline $\begin{array}{l}\text { C242 } \\
119.1(5)\end{array}$ & C241 & $\mathrm{H} 241$ & $120.4(5)$ & F322 & C322 & C321 \\
\hline $\begin{array}{l}\text { C240 } \\
124.9(7)\end{array}$ & C241 & $\mathrm{H} 241$ & $120.4(5)$ & C323 & C322 & C321 \\
\hline $\begin{array}{l}\text { C324 } \\
112.5(4)\end{array}$ & C323 & F323 & $120.2(6)$ & $\mathrm{C} 412$ & C411 & $\mathrm{C} 416$ \\
\hline $\begin{array}{l}\text { C324 } \\
120.8(4)\end{array}$ & C323 & C322 & $120.5(6)$ & $\mathrm{C} 412$ & C411 & B4 \\
\hline $\begin{array}{l}\text { F323 } \\
126.5(4)\end{array}$ & C 323 & C322 & 119.1(7) & $\mathrm{C} 416$ & C411 & B4 \\
\hline $\begin{array}{l}\text { F324 } \\
115.8(4)\end{array}$ & C324 & C323 & $120.8(7)$ & F412 & C412 & C413 \\
\hline $\begin{array}{l}\text { F324 } \\
119.3(5)\end{array}$ & C324 & C325 & $119.9(7)$ & F412 & C412 & C411 \\
\hline $\begin{array}{l}\text { C323 } \\
124.9(5)\end{array}$ & C 324 & C325 & $119.3(6)$ & C413 & C412 & C411 \\
\hline $\begin{array}{l}\text { F325 } \\
119.4(5)\end{array}$ & C 325 & C324 & $120.6(6)$ & F413 & C413 & C414 \\
\hline $\begin{array}{l}\text { F325 } \\
121.0(5)\end{array}$ & C325 & C326 & $120.6(6)$ & F413 & $\mathrm{C} 413$ & $\mathrm{C} 412$ \\
\hline $\begin{array}{l}\text { C324 } \\
119.7(5)\end{array}$ & C 325 & C326 & $118.8(6)$ & C414 & C413 & C412 \\
\hline $\begin{array}{l}\text { F326 } \\
120.9(5)\end{array}$ & C 326 & C 325 & $115.9(5)$ & F414 & C414 & C413 \\
\hline $\begin{array}{l}\text { F326 } \\
120.4(5)\end{array}$ & C326 & C321 & $119.8(4)$ & F414 & C414 & C415 \\
\hline $\begin{array}{l}\text { C325 } \\
118.7(5)\end{array}$ & C 326 & C321 & $124.3(6)$ & $\mathrm{C} 413$ & C414 & $\mathrm{C} 415$ \\
\hline $\begin{array}{l}\text { C336 } \\
119.9(4)\end{array}$ & C331 & C332 & $112.0(4)$ & F415 & $\mathrm{C} 415$ & C416 \\
\hline $\begin{array}{l}\text { C336 } \\
120.0(5)\end{array}$ & C331 & B3 & $121.3(4)$ & F415 & C415 & C414 \\
\hline $\begin{array}{l}\text { C332 } \\
120.0(5)\end{array}$ & C331 & B3 & $126.5(4)$ & C416 & C415 & C414 \\
\hline F332 & C332 & C333 & $114.1(4)$ & F416 & $\mathrm{C} 416$ & $\mathrm{C} 415$ \\
\hline
\end{tabular}




\begin{tabular}{|c|c|c|c|c|c|c|}
\hline $\begin{array}{l}115.8(4) \\
\text { F332 }\end{array}$ & C 332 & C331 & $120.9(4)$ & F416 & $\mathrm{C} 416$ & C411 \\
\hline $\begin{array}{l}120.1(4) \\
\text { C333 }\end{array}$ & C 332 & C331 & $125.0(4)$ & C415 & $\mathrm{C} 416$ & C411 \\
\hline $\begin{array}{l}124.1(4) \\
\text { F333 } \\
112.3(5)\end{array}$ & C 333 & C332 & $121.2(4)$ & $\mathrm{C} 426$ & $\mathrm{C} 421$ & $\mathrm{C} 422$ \\
\hline $\begin{array}{l}\text { F333 } \\
121.6(5)\end{array}$ & C333 & C334 & $119.8(4)$ & $\mathrm{C} 426$ & $\mathrm{C} 421$ & B4 \\
\hline $\begin{array}{l}\text { C332 } \\
125.3(5)\end{array}$ & C 333 & C334 & $119.0(5)$ & $\mathrm{C} 422$ & $\mathrm{C} 421$ & B4 \\
\hline $\begin{array}{l}\text { F334 } \\
115.3(5)\end{array}$ & C334 & C335 & $121.5(5)$ & F422 & $\mathrm{C} 422$ & $\mathrm{C} 423$ \\
\hline $\begin{array}{l}\text { F334 } \\
120.4(5)\end{array}$ & C334 & $\mathrm{C} 333$ & $119.9(5)$ & F422 & $\mathrm{C} 422$ & C421 \\
\hline $\begin{array}{l}\text { C335 } \\
124.4(5)\end{array}$ & C334 & C333 & $118.6(5)$ & $\mathrm{C} 423$ & $\mathrm{C} 422$ & C421 \\
\hline $\begin{array}{l}\text { F335 } \\
120.3(5)\end{array}$ & C335 & C334 & $119.1(5)$ & F423 & $\mathrm{C} 423$ & C424 \\
\hline $\begin{array}{l}\text { F335 } \\
119.7(5)\end{array}$ & $\mathrm{C} 335$ & C336 & $120.8(5)$ & F423 & $\mathrm{C} 423$ & $\mathrm{C} 422$ \\
\hline $\begin{array}{l}\text { C334 } \\
120.0(5)\end{array}$ & C335 & C336 & $120.1(5)$ & $\mathrm{C} 424$ & $\mathrm{C} 423$ & $\mathrm{C} 422$ \\
\hline $\begin{array}{l}\text { F336 } \\
121.0(5)\end{array}$ & C336 & C335 & $115.8(4)$ & F424 & $\mathrm{C} 424$ & $\mathrm{C} 423$ \\
\hline $\begin{array}{l}\text { F336 } \\
120.1(6)\end{array}$ & C336 & C331 & $119.0(5)$ & F424 & $\mathrm{C} 424$ & C425 \\
\hline $\begin{array}{l}\text { C335 } \\
118.9(5)\end{array}$ & C336 & C331 & $125.2(5)$ & $\mathrm{C} 423$ & $\mathrm{C} 424$ & C425 \\
\hline $\begin{array}{l}\text { C346 } \\
120.8(5)\end{array}$ & C341 & C342 & $112.0(5)$ & F425 & $\mathrm{C} 425$ & C426 \\
\hline $\begin{array}{l}\text { C346 } \\
119.9(5)\end{array}$ & C341 & B3 & $126.5(5)$ & F425 & $\mathrm{C} 425$ & C424 \\
\hline $\begin{array}{l}\text { C342 } \\
119.3(5)\end{array}$ & C341 & B3 & $120.8(5)$ & $\mathrm{C} 426$ & $\mathrm{C} 425$ & C424 \\
\hline $\begin{array}{l}\text { F342 } \\
115.9(5)\end{array}$ & C342 & C343 & $116.7(5)$ & F426 & $\mathrm{C} 426$ & $\mathrm{C} 425$ \\
\hline $\begin{array}{l}\text { F342 } \\
119.1(5)\end{array}$ & C 342 & C341 & $119.0(5)$ & F426 & $\mathrm{C} 426$ & C421 \\
\hline $\begin{array}{l}\mathrm{C} 343 \\
125.0(5)\end{array}$ & C342 & $\mathrm{C} 341$ & $124.3(5)$ & $\mathrm{C} 425$ & $\mathrm{C} 426$ & C421 \\
\hline $\begin{array}{l}\text { F343 } \\
113.3(5)\end{array}$ & C343 & C342 & $120.8(5)$ & $\mathrm{C} 432$ & $\mathrm{C} 431$ & C436 \\
\hline $\begin{array}{l}\text { F343 } \\
126.6(5)\end{array}$ & C343 & C344 & $118.9(5)$ & $\mathrm{C} 432$ & $\mathrm{C} 431$ & B4 \\
\hline $\begin{array}{l}\text { C342 } \\
119.6(5)\end{array}$ & C343 & C344 & $120.2(5)$ & $\mathrm{C} 436$ & $\mathrm{C} 431$ & B4 \\
\hline $\begin{array}{l}\text { F344 } \\
121.6(5)\end{array}$ & C344 & C345 & $121.5(5)$ & F432 & $\mathrm{C} 432$ & C431 \\
\hline $\begin{array}{l}\text { F344 } \\
114.5(5)\end{array}$ & C344 & C343 & $120.4(6)$ & F432 & $\mathrm{C} 432$ & C433 \\
\hline $\begin{array}{l}\mathrm{C} 345 \\
123.8(6)\end{array}$ & C344 & C343 & 118.1(6) & C431 & $\mathrm{C} 432$ & C433 \\
\hline $\begin{array}{l}\text { C344 } \\
119.6(6)\end{array}$ & C345 & F345 & $119.6(5)$ & $\mathrm{C} 434$ & $\mathrm{C} 433$ & F433 \\
\hline C344 & C345 & C346 & $120.4(5)$ & C434 & C433 & C432 \\
\hline
\end{tabular}




\begin{tabular}{|c|c|c|c|c|c|c|}
\hline $\begin{array}{l}120.4(6) \\
\text { F345 }\end{array}$ & $\mathrm{C} 345$ & C346 & $120.0(5)$ & F433 & $\mathrm{C} 433$ & $\mathrm{C} 432$ \\
\hline $\begin{array}{l}120.0(6) \\
\text { F346 } \\
121.0(6)\end{array}$ & C346 & C 341 & $120.3(5)$ & F434 & $\mathrm{C} 434$ & $\mathrm{C} 433$ \\
\hline $\begin{array}{l}\text { F346 } \\
120.2(6)\end{array}$ & C346 & $\mathrm{C} 345$ & $114.8(5)$ & F434 & $\mathrm{C} 434$ & $\mathrm{C} 435$ \\
\hline $\begin{array}{l}\text { C341 } \\
118.8(6)\end{array}$ & C346 & C345 & $124.9(5)$ & $\mathrm{C} 433$ & $\mathrm{C} 434$ & $\mathrm{C} 435$ \\
\hline $\begin{array}{l}\text { F435 } \\
115.9(11)\end{array}$ & C435 & $\mathrm{C} 436$ & $120.4(6)$ & $\mathrm{Cl} 94$ & C934 & $\mathrm{Cl} 93$ \\
\hline $\begin{array}{l}\text { F435 } \\
108.3(11)\end{array}$ & C435 & $\mathrm{C} 434$ & $120.8(6)$ & $\mathrm{Cl} 94$ & C934 & H93A \\
\hline $\begin{array}{l}\text { C436 } \\
108.3(11)\end{array}$ & $\mathrm{C} 435$ & $\mathrm{C} 434$ & $118.8(6)$ & $\mathrm{Cl} 93$ & C934 & H93A \\
\hline $\begin{array}{l}\text { F436 } \\
108.3(11)\end{array}$ & $\mathrm{C} 436$ & $\mathrm{C} 435$ & $116.1(5)$ & $\mathrm{Cl} 94$ & C934 & H93B \\
\hline $\begin{array}{l}\text { F436 } \\
108.3(11)\end{array}$ & $\mathrm{C} 436$ & $\mathrm{C} 431$ & $119.0(5)$ & $\mathrm{Cl} 93$ & C934 & H93B \\
\hline $\begin{array}{l}\text { C435 } \\
107.4(11)\end{array}$ & $\mathrm{C} 436$ & $\mathrm{C} 431$ & $124.8(6)$ & H93A & C934 & H93B \\
\hline $\begin{array}{l}\text { C446 } \\
116.5(6)\end{array}$ & $\mathrm{C} 441$ & C442 & $114.9(5)$ & $\mathrm{C} 195$ & C956 & $\mathrm{Cl} 96$ \\
\hline $\begin{array}{l}\text { C446 } \\
108.2(6)\end{array}$ & C441 & B4 & $126.8(5)$ & $\mathrm{Cl} 95$ & C956 & H95A \\
\hline $\begin{array}{l}\mathrm{C} 442 \\
108.2(6)\end{array}$ & $\mathrm{C} 441$ & B4 & $118.2(5)$ & $\mathrm{Cl} 96$ & C956 & H95A \\
\hline $\begin{array}{l}\text { F442 } \\
108.2(6)\end{array}$ & $\mathrm{C} 442$ & C443 & $116.0(5)$ & $\mathrm{Cl} 95$ & C956 & H95B \\
\hline $\begin{array}{l}\text { F442 } \\
108.2(6)\end{array}$ & $\mathrm{C} 442$ & C441 & $120.7(4)$ & $\mathrm{Cl} 96$ & C956 & H95B \\
\hline $\begin{array}{l}\text { C443 } \\
107.3(6)\end{array}$ & $\mathrm{C} 442$ & C441 & $123.3(5)$ & H95A & C956 & H95B \\
\hline $\begin{array}{l}\text { F443 } \\
120.3(12)\end{array}$ & C443 & C442 & $121.4(6)$ & $\mathrm{C} 197$ & C978 & $\mathrm{Cl} 98$ \\
\hline $\begin{array}{l}\text { F443 } \\
107.2(12)\end{array}$ & C443 & C444 & $118.7(5)$ & $\mathrm{Cl} 97$ & C978 & H97A \\
\hline $\begin{array}{l}\text { C442 } \\
107.2(12)\end{array}$ & C443 & C444 & $119.9(6)$ & $\mathrm{C} 198$ & C978 & H97A \\
\hline $\begin{array}{l}\text { F444 } \\
107.2(12)\end{array}$ & C444 & C445 & $121.1(6)$ & $\mathrm{Cl} 97$ & C978 & H97B \\
\hline $\begin{array}{l}\text { F444 } \\
107.2(12)\end{array}$ & C444 & C443 & $120.6(7)$ & $\mathrm{Cl} 98$ & C978 & H97B \\
\hline $\begin{array}{l}\text { C445 } \\
106.9(12)\end{array}$ & C444 & C443 & $118.3(6)$ & H97A & C978 & H97B \\
\hline $\begin{array}{l}\text { C444 } \\
114.7(4)\end{array}$ & $\mathrm{C} 445$ & F445 & $120.4(6)$ & C331 & B3 & C321 \\
\hline $\begin{array}{l}\text { C444 } \\
101.4(4)\end{array}$ & $\mathrm{C} 445$ & C446 & $120.8(6)$ & C331 & B3 & C341 \\
\hline $\begin{array}{l}\text { F445 } \\
113.1(5)\end{array}$ & $\mathrm{C} 445$ & C446 & $118.8(7)$ & C321 & B3 & C341 \\
\hline $\begin{array}{l}\text { F446 } \\
114.2(4)\end{array}$ & $\mathrm{C} 446$ & C441 & $121.8(5)$ & $\mathrm{C} 331$ & B3 & C311 \\
\hline $\begin{array}{l}\text { F446 } \\
101.3(4)\end{array}$ & C446 & C445 & $115.4(5)$ & C321 & B3 & C311 \\
\hline C441 & C446 & C445 & $122.8(6)$ & C341 & B3 & C311 \\
\hline
\end{tabular}




$\begin{array}{lllllll}112.7(4) & & & & & \\ \text { C192 } & \text { C912 } & \text { C191 } & 118.2(8) & \text { C411 } & \text { B4 } & \text { C421 } \\ \begin{array}{l}101.5(4) \\ \text { C192 }\end{array} & \text { C912 } & \text { H91A } & 107.8(8) & \text { C411 } & \text { B4 } & \text { C431 } \\ \begin{array}{l}113.8(4) \\ \text { C191 }\end{array} & \text { C912 } & \text { H91A } & 107.8(8) & \text { C421 } & \text { B4 } & \text { C431 } \\ \begin{array}{l}113.2(4) \\ \text { C192 }\end{array} & \text { C912 } & \text { H91B } & 107.8(8) & \text { C411 } & \text { B4 } & \text { C441 } \\ \begin{array}{l}114.6(4) \\ \text { C191 }\end{array} & \text { C912 } & \text { H91B } & 107.8(8) & \text { C421 } & \text { B4 } & \text { C441 } \\ 113.8(4) & & & & & & \\ \text { H91A } & \text { C912 } & \text { H91B } & 107.1(8) & \text { C431 } & \text { B4 } & \text { C441 } \\ 100.4(4) & & & & & & \end{array}$

Numbers in parentheses are estimated standard deviations in the least significant digits.

Table S6. Bond Distances in Angstroms for [Re(NMes) $\left(\mathrm{PPh}_{3}\right)$ saldach $\left.{ }^{+}\right], 7 a$

\begin{tabular}{|c|c|c|c|c|c|}
\hline Atom 1 & Atom 2 & Distance & Atom 1 & Atom 2 & Distance \\
\hline Re1 & N120 & $1.755(4)$ & F414 & C414 & $1.352(6)$ \\
\hline Re1 & O119 & $1.986(4)$ & F415 & C415 & $1.355(6)$ \\
\hline Re1 & O11 & $2.027(3)$ & F416 & C416 & $1.353(5)$ \\
\hline Re1 & N112 & $2.091(4)$ & F422 & C422 & $1.350(6)$ \\
\hline Re1 & N18 & $2.126(4)$ & F423 & $\mathrm{C} 423$ & $1.349(6)$ \\
\hline Re1 & P130 & $2.4644(14)$ & F424 & C424 & $1.353(6)$ \\
\hline $\operatorname{Re} 2$ & N220 & $1.721(4)$ & F425 & $\mathrm{C} 425$ & $1.350(6)$ \\
\hline Re2 & O219 & $2.008(4)$ & F426 & C426 & $1.358(5)$ \\
\hline Re2 & $\mathrm{O} 21$ & $2.027(3)$ & F432 & C432 & $1.356(6)$ \\
\hline Re2 & N212 & $2.082(4)$ & F433 & C433 & $1.355(7)$ \\
\hline $\operatorname{Re} 2$ & N28 & $2.156(5)$ & F434 & C434 & $1.341(7)$ \\
\hline Re2 & P230 & $2.4670(14)$ & F435 & C435 & $1.329(7)$ \\
\hline $\mathrm{Cl} 91$ & C912 & $1.687(11)$ & F436 & C436 & $1.353(6)$ \\
\hline $\mathrm{Cl} 92$ & C912 & $1.673(10)$ & F442 & C442 & $1.340(6)$ \\
\hline $\mathrm{Cl} 193$ & C934 & $1.619(12)$ & F443 & C443 & $1.351(7)$ \\
\hline $\mathrm{Cl} 94$ & C934 & $1.604(12)$ & F444 & C444 & $1.350(6)$ \\
\hline $\mathrm{C} 195$ & C956 & $1.686(9)$ & F445 & C445 & $1.362(7)$ \\
\hline $\mathrm{Cl} 96$ & C956 & $1.712(9)$ & F446 & C446 & $1.357(7)$ \\
\hline $\mathrm{Cl} 97$ & C978 & $1.581(15)$ & O11 & C11 & $1.322(6)$ \\
\hline $\mathrm{Cl} 98$ & C978 & $1.599(16)$ & $\mathrm{O} 21$ & C21 & $1.338(6)$ \\
\hline P130 & C131 & $1.810(5)$ & O119 & C119 & $1.327(6)$ \\
\hline P130 & C137 & $1.818(5)$ & O219 & C219 & $1.337(6)$ \\
\hline P130 & C143 & $1.836(5)$ & N18 & C17 & $1.269(7)$ \\
\hline P230 & $\mathrm{C} 231$ & $1.800(5)$ & N18 & C19 & $1.476(7)$ \\
\hline P230 & $\mathrm{C} 243$ & $1.822(5)$ & N28 & $\mathrm{C} 27$ & $1.269(7)$ \\
\hline P230 & $\mathrm{C} 237$ & $1.822(5)$ & N28 & $\mathrm{C} 29$ & $1.471(7)$ \\
\hline F312 & C312 & $1.350(6)$ & N112 & C113 & $1.299(7)$ \\
\hline F313 & C313 & $1.340(7)$ & N112 & C111 & $1.502(7)$ \\
\hline F314 & C314 & $1.353(7)$ & N120 & $\mathrm{C} 121$ & $1.358(7)$ \\
\hline F315 & C315 & $1.343(7)$ & N212 & $\mathrm{C} 213$ & $1.300(8)$ \\
\hline F316 & C316 & $1.358(6)$ & N212 & $\mathrm{C} 211$ & $1.492(7)$ \\
\hline F322 & C322 & $1.361(7)$ & N220 & $\mathrm{C} 221$ & $1.375(7)$ \\
\hline F323 & C323 & $1.364(8)$ & C11 & C12 & $1.405(7)$ \\
\hline F324 & C324 & $1.352(6)$ & C11 & C16 & $1.424(7)$ \\
\hline F325 & C325 & $1.347(7)$ & C12 & C13 & $1.394(7)$ \\
\hline F326 & C326 & $1.349(6)$ & $\mathrm{C} 12$ & H12 & $0.950(7)$ \\
\hline
\end{tabular}




\begin{tabular}{|c|c|c|c|c|c|}
\hline F332 & C332 & $1.356(5)$ & $\mathrm{C} 13$ & $\mathrm{C} 14$ & $1.391(9)$ \\
\hline F333 & C333 & $1.341(6)$ & $\mathrm{C} 13$ & H13 & $0.950(9)$ \\
\hline F334 & C334 & $1.345(6)$ & C14 & $\mathrm{C} 15$ & $1.385(9)$ \\
\hline F335 & C335 & $1.355(6)$ & C14 & H14 & $0.950(9)$ \\
\hline F336 & C336 & $1.359(6)$ & $\mathrm{C} 15$ & $\mathrm{C} 16$ & $1.412(8)$ \\
\hline F342 & C342 & $1.351(6)$ & $\mathrm{C} 15$ & H15 & $0.950(8)$ \\
\hline F343 & C343 & $1.356(6)$ & $\mathrm{C} 16$ & $\mathrm{C} 17$ & $1.432(8)$ \\
\hline F344 & C344 & $1.346(7)$ & $\mathrm{C} 17$ & H17 & $0.950(8)$ \\
\hline F345 & C345 & $1.358(6)$ & C19 & C110 & $1.582(8)$ \\
\hline F346 & C346 & $1.360(6)$ & C19 & H19A & $0.990(8)$ \\
\hline F412 & C412 & $1.350(6)$ & C19 & H19B & $0.990(8)$ \\
\hline F413 & $\mathrm{C} 413$ & $1.344(6)$ & $\mathrm{C} 21$ & C22 & $1.384(7)$ \\
\hline C21 & $\mathrm{C} 26$ & $1.422(7)$ & C129 & $\mathrm{H} 12 \mathrm{G}$ & $0.980(7)$ \\
\hline $\mathrm{C} 22$ & $\mathrm{C} 23$ & $1.385(7)$ & C129 & $\mathrm{H} 12 \mathrm{H}$ & $0.980(7)$ \\
\hline $\mathrm{C} 22$ & $\mathrm{H} 22$ & $0.950(7)$ & C129 & H12I & $0.980(7)$ \\
\hline $\mathrm{C} 23$ & $\mathrm{C} 24$ & $1.381(9)$ & C131 & C132 & $1.397(7)$ \\
\hline $\mathrm{C} 23$ & $\mathrm{H} 23$ & $0.950(9)$ & C131 & C136 & $1.401(7)$ \\
\hline $\mathrm{C} 24$ & $\mathrm{C} 25$ & $1.383(9)$ & C132 & $\mathrm{C} 133$ & $1.386(7)$ \\
\hline C24 & $\mathrm{H} 24$ & $0.950(9)$ & C132 & H132 & $0.950(7)$ \\
\hline $\mathrm{C} 25$ & $\mathrm{C} 26$ & $1.402(8)$ & C133 & C134 & $1.370(7)$ \\
\hline $\mathrm{C} 25$ & $\mathrm{H} 25$ & $0.950(8)$ & C133 & H133 & $0.950(7)$ \\
\hline $\mathrm{C} 26$ & $\mathrm{C} 27$ & $1.429(8)$ & C134 & C135 & $1.385(8)$ \\
\hline $\mathrm{C} 27$ & H27 & $0.950(8)$ & C134 & H134 & $0.950(8)$ \\
\hline C29 & C210 & $1.541(9)$ & C135 & C136 & $1.379(7)$ \\
\hline C29 & $\mathrm{H} 29 \mathrm{~A}$ & $0.990(9)$ & C135 & H135 & $0.950(7)$ \\
\hline C29 & H29B & $0.990(9)$ & C136 & H136 & $0.950(7)$ \\
\hline C110 & C111 & $1.501(8)$ & C137 & $\mathrm{C} 142$ & $1.397(7)$ \\
\hline C110 & H11A & $0.990(8)$ & C137 & $\mathrm{C} 138$ & $1.403(8)$ \\
\hline C110 & H11B & $0.990(8)$ & C138 & C139 & $1.396(8)$ \\
\hline C111 & $\mathrm{H} 11 \mathrm{C}$ & $0.990(8)$ & C138 & H138 & $0.950(8)$ \\
\hline C111 & H11D & $0.990(8)$ & C139 & $\mathrm{C} 140$ & $1.356(9)$ \\
\hline C113 & C114 & $1.425(8)$ & C139 & H139 & $0.950(9)$ \\
\hline C113 & H113 & $0.950(8)$ & C140 & C141 & $1.400(9)$ \\
\hline C114 & C119 & $1.409(7)$ & C140 & H140 & $0.950(9)$ \\
\hline C114 & C115 & $1.411(8)$ & C141 & $\mathrm{C} 142$ & $1.382(8)$ \\
\hline C115 & C116 & $1.352(9)$ & C141 & H141 & $0.950(8)$ \\
\hline C115 & H115 & $0.950(9)$ & C142 & H142 & $0.950(8)$ \\
\hline C116 & C117 & $1.404(9)$ & C143 & C144 & $1.380(7)$ \\
\hline C116 & H116 & $0.950(9)$ & C143 & C148 & $1.394(7)$ \\
\hline C117 & C118 & $1.379(9)$ & C144 & C145 & $1.392(8)$ \\
\hline C117 & H117 & $0.950(9)$ & C144 & H144 & $0.950(8)$ \\
\hline C118 & C119 & $1.405(8)$ & C145 & C146 & $1.366(9)$ \\
\hline C118 & H118 & $0.950(8)$ & C145 & H145 & $0.950(9)$ \\
\hline C121 & C122 & $1.422(7)$ & C146 & C147 & $1.358(8)$ \\
\hline $\mathrm{C} 121$ & C126 & $1.429(8)$ & C146 & H146 & $0.950(8)$ \\
\hline C122 & C123 & $1.381(8)$ & C147 & C148 & $1.385(7)$ \\
\hline C122 & C127 & $1.498(8)$ & C147 & H147 & $0.950(7)$ \\
\hline $\mathrm{C} 123$ & $\mathrm{C} 124$ & $1.367(9)$ & C148 & H148 & $0.950(7)$ \\
\hline C123 & H123 & $0.950(9)$ & C210 & $\mathrm{C} 211$ & $1.510(10)$ \\
\hline C124 & C125 & $1.397(8)$ & $\mathrm{C} 210$ & $\mathrm{H} 21 \mathrm{~A}$ & $0.990(10)$ \\
\hline C124 & C128 & $1.516(8)$ & $\mathrm{C} 210$ & $\mathrm{H} 21 \mathrm{~B}$ & $0.990(10)$ \\
\hline C125 & C126 & $1.377(8)$ & $\mathrm{C} 211$ & $\mathrm{H} 21 \mathrm{C}$ & $0.990(10)$ \\
\hline C125 & H125 & $0.950(8)$ & $\mathrm{C} 211$ & H21D & $0.990(10)$ \\
\hline C126 & C129 & $1.507(7)$ & C213 & C214 & $1.430(8)$ \\
\hline C127 & $\mathrm{H} 12 \mathrm{~A}$ & $0.980(7)$ & $\mathrm{C} 213$ & $\mathrm{H} 213$ & $0.950(8)$ \\
\hline C127 & H12B & $0.980(7)$ & C214 & $\mathrm{C} 215$ & $1.403(9)$ \\
\hline
\end{tabular}




\begin{tabular}{|c|c|c|c|c|c|}
\hline C127 & $\mathrm{H} 12 \mathrm{C}$ & $0.980(7)$ & C214 & C219 & $1.406(8)$ \\
\hline $\mathrm{C} 128$ & H12D & $0.980(7)$ & $\mathrm{C} 215$ & $\mathrm{C} 216$ & $1.347(10)$ \\
\hline C128 & H12E & $0.980(7)$ & $\mathrm{C} 215$ & H215 & $0.950(10)$ \\
\hline C128 & $\mathrm{H} 12 \mathrm{~F}$ & $0.980(7)$ & C217 & C218 & $1.373(9)$ \\
\hline C217 & C216 & $1.406(9)$ & $\mathrm{C} 244$ & $\mathrm{C} 245$ & $1.388(8)$ \\
\hline C217 & H217 & $0.950(9)$ & C244 & $\mathrm{H} 244$ & $0.950(8)$ \\
\hline C218 & C219 & $1.398(8)$ & $\mathrm{C} 245$ & C246 & $1.361(9)$ \\
\hline C218 & H218 & $0.950(8)$ & C245 & H245 & $0.950(9)$ \\
\hline $\mathrm{C} 221$ & $\mathrm{C} 226$ & $1.414(7)$ & $\mathrm{C} 246$ & $\mathrm{C} 247$ & $1.403(9)$ \\
\hline C221 & $\mathrm{C} 222$ & $1.417(7)$ & $\mathrm{C} 246$ & $\mathrm{H} 246$ & $0.950(9)$ \\
\hline C222 & $\mathrm{C} 223$ & $1.361(8)$ & C247 & C248 & $1.386(7)$ \\
\hline C222 & $\mathrm{C} 227$ & $1.496(7)$ & C247 & H247 & $0.950(7)$ \\
\hline C223 & $\mathrm{C} 224$ & $1.392(8)$ & $\mathrm{C} 248$ & $\mathrm{H} 248$ & $0.950(7)$ \\
\hline C223 & $\mathrm{H} 223$ & $0.950(8)$ & C216 & H216 & $0.950(7)$ \\
\hline C224 & C225 & $1.371(9)$ & C311 & C316 & $1.390(8)$ \\
\hline C224 & $\mathrm{C} 228$ & $1.531(8)$ & C311 & C312 & $1.398(7)$ \\
\hline $\mathrm{C} 225$ & $\mathrm{C} 226$ & $1.405(8)$ & C311 & B3 & $1.657(8)$ \\
\hline $\mathrm{C} 225$ & $\mathrm{H} 225$ & $0.950(8)$ & C 312 & $\mathrm{C} 313$ & $1.370(8)$ \\
\hline C226 & C229 & $1.493(9)$ & C313 & C314 & $1.362(9)$ \\
\hline C227 & $\mathrm{H} 22 \mathrm{~A}$ & $0.980(9)$ & C314 & C315 & $1.373(9)$ \\
\hline C227 & H22B & $0.980(9)$ & C315 & C316 & $1.375(8)$ \\
\hline C227 & $\mathrm{H} 22 \mathrm{C}$ & $0.980(9)$ & C321 & C322 & $1.377(8)$ \\
\hline C228 & H22D & $0.980(9)$ & C321 & C326 & $1.429(8)$ \\
\hline C228 & $\mathrm{H} 22 \mathrm{E}$ & $0.980(9)$ & C321 & B3 & $1.639(7)$ \\
\hline C228 & $\mathrm{H} 22 \mathrm{~F}$ & $0.980(9)$ & C322 & C323 & $1.372(8)$ \\
\hline C229 & $\mathrm{H} 22 \mathrm{G}$ & $0.980(9)$ & C323 & C324 & $1.357(10)$ \\
\hline C229 & $\mathrm{H} 22 \mathrm{H}$ & $0.980(9)$ & C324 & C325 & $1.376(10)$ \\
\hline C229 & H22I & $0.980(9)$ & C325 & C326 & $1.381(7)$ \\
\hline C231 & $\mathrm{C} 232$ & $1.394(7)$ & C331 & C336 & $1.385(6)$ \\
\hline C231 & $\mathrm{C} 236$ & $1.412(8)$ & C331 & C332 & $1.393(7)$ \\
\hline C232 & C233 & $1.374(8)$ & C331 & B3 & $1.639(7)$ \\
\hline $\mathrm{C} 232$ & H232 & $0.950(8)$ & C332 & C333 & $1.379(7)$ \\
\hline $\mathrm{C} 233$ & C234 & $1.390(10)$ & C333 & C334 & $1.382(7)$ \\
\hline C233 & $\mathrm{H} 233$ & $0.950(10)$ & C334 & C335 & $1.361(8)$ \\
\hline C234 & $\mathrm{C} 235$ & $1.382(10)$ & C335 & C336 & $1.367(7)$ \\
\hline C234 & $\mathrm{H} 234$ & $0.950(10)$ & C341 & C346 & $1.376(7)$ \\
\hline $\mathrm{C} 235$ & C236 & $1.401(9)$ & C341 & C342 & $1.410(7)$ \\
\hline C235 & H235 & $0.950(9)$ & C341 & B3 & $1.647(8)$ \\
\hline C236 & H236 & $0.950(9)$ & C342 & C343 & $1.359(8)$ \\
\hline C237 & C238 & $1.374(7)$ & C343 & C344 & $1.381(8)$ \\
\hline $\mathrm{C} 237$ & C242 & $1.397(7)$ & C344 & C345 & $1.348(8)$ \\
\hline C238 & C239 & $1.385(7)$ & C345 & C346 & $1.381(8)$ \\
\hline C238 & H238 & $0.950(7)$ & C411 & C412 & $1.385(7)$ \\
\hline C239 & C240 & $1.365(7)$ & C411 & C416 & $1.408(7)$ \\
\hline C239 & H239 & $0.950(7)$ & C411 & B4 & $1.649(8)$ \\
\hline C240 & C241 & $1.386(8)$ & C412 & C413 & $1.369(8)$ \\
\hline C240 & $\mathrm{H} 240$ & $0.950(8)$ & C413 & C414 & $1.367(8)$ \\
\hline C241 & C242 & $1.371(8)$ & C414 & C415 & $1.376(7)$ \\
\hline $\mathrm{C} 241$ & $\mathrm{H} 241$ & $0.950(8)$ & $\mathrm{C} 415$ & $\mathrm{C} 416$ & $1.361(7)$ \\
\hline $\mathrm{C} 242$ & H242 & $0.950(8)$ & C421 & $\mathrm{C} 426$ & $1.391(7)$ \\
\hline C243 & C244 & $1.385(7)$ & C421 & C422 & $1.391(7)$ \\
\hline C243 & C248 & $1.397(7)$ & C421 & B4 & $1.659(8)$ \\
\hline C422 & $\mathrm{C} 423$ & $1.379(8)$ & C441 & B4 & $1.666(7)$ \\
\hline C423 & C424 & $1.357(8)$ & C442 & C443 & $1.374(7)$ \\
\hline C424 & C425 & $1.374(8)$ & C443 & C444 & $1.379(9)$ \\
\hline C425 & C426 & $1.372(8)$ & C444 & C445 & $1.352(9)$ \\
\hline
\end{tabular}




$\begin{array}{llllll}\text { C431 } & \text { C432 } & 1.365(8) & \text { C445 } & \text { C446 } & 1.393(8) \\ \text { C431 } & \text { C436 } & 1.394(8) & \text { C912 } & \text { H91A } & 0.990(8) \\ \text { C431 } & \text { B4 } & 1.666(8) & \text { C912 } & \text { H91B } & 0.990(8) \\ \text { C432 } & \text { C433 } & 1.394(8) & \text { C934 } & \text { H93A } & 0.990(8) \\ \text { C433 } & \text { C434 } & 1.354(9) & \text { C934 } & \text { H93B } & 0.990(8) \\ \text { C434 } & \text { C435 } & 1.380(9) & \text { C956 } & \text { H95A } & 0.990(8) \\ \text { C435 } & \text { C436 } & 1.377(8) & \text { C956 } & \text { H95B } & 0.990(8) \\ \text { C441 } & \text { C446 } & 1.366(8) & \text { C978 } & \text { H97A } & 0.990(8) \\ \text { C441 } & \text { C442 } & 1.389(8) & \text { C978 } & \text { H97B } & 0.990(8)\end{array}$

Numbers in parentheses are estimated standard deviations in the least

Table S7. Torsional Angles in Degrees for $\left[\mathrm{Re}(\mathrm{NMes})\left(\mathrm{PPh}_{3}\right) \mathrm{saldach}^{+}\right], 7 \mathbf{a}$

\begin{tabular}{|c|c|c|c|c|}
\hline Atom 1 & Atom 2 & Atom 3 & Atom 4 & Angle \\
\hline$\overline{\mathrm{O}(11)}$ & $\overline{\operatorname{Re}(1)}$ & $\overline{P(130)}$ & $\overline{C(131)}$ & $14 \overline{46.30}(0.19)$ \\
\hline $\mathrm{O}(11)$ & $\operatorname{Re}(1)$ & $\mathrm{P}(130)$ & $C(137)$ & $-93.31(0.20)$ \\
\hline $\mathrm{O}(11)$ & $\operatorname{Re}(1)$ & $\mathrm{P}(130)$ & $C(143)$ & $24.25(0.22)$ \\
\hline$O(119)$ & $\operatorname{Re}(1)$ & $\mathrm{P}(130)$ & $\mathrm{C}(131)$ & $65.16(0.19)$ \\
\hline $\mathrm{O}(119)$ & $\operatorname{Re}(1)$ & $\mathrm{P}(130)$ & $C(137)$ & $-174.45(0.20)$ \\
\hline $\mathrm{O}(119)$ & $\operatorname{Re}(1)$ & $\mathrm{P}(130)$ & $C(143)$ & $-56.89(0.22)$ \\
\hline $\mathrm{N}(18)$ & $\operatorname{Re}(1)$ & $\mathrm{P}(130)$ & $C(131)$ & $103.04(1.20)$ \\
\hline $\mathrm{N}(18)$ & $\operatorname{Re}(1)$ & $\mathrm{P}(130)$ & $C(137)$ & $-136.56(1.19)$ \\
\hline $\mathrm{N}(18)$ & $\operatorname{Re}(1)$ & $\mathrm{P}(130)$ & $C(143)$ & $-19.01(1.22)$ \\
\hline $\mathrm{N}(112)$ & $\operatorname{Re}(1)$ & $\mathrm{P}(130)$ & $C(131)$ & $-16.49(0.20)$ \\
\hline $\mathrm{N}(112)$ & $\operatorname{Re}(1)$ & $\mathrm{P}(130)$ & $C(137)$ & $103.91(0.22)$ \\
\hline $\mathrm{N}(112)$ & $\operatorname{Re}(1)$ & $\mathrm{P}(130)$ & $C(143)$ & $-138.53(0.23)$ \\
\hline $\mathrm{N}(120)$ & $\operatorname{Re}(1)$ & $\mathrm{P}(130)$ & $C(131)$ & $-113.73(0.23)$ \\
\hline $\mathrm{N}(120)$ & $\operatorname{Re}(1)$ & $\mathrm{P}(130)$ & $C(137)$ & $6.66(0.24)$ \\
\hline $\mathrm{N}(120)$ & $\operatorname{Re}(1)$ & $\mathrm{P}(130)$ & $C(143)$ & $124.22(0.25)$ \\
\hline $\mathrm{P}(130)$ & $\operatorname{Re}(1)$ & $\mathrm{O}(11)$ & $\mathrm{C}(11)$ & $-143.74(0.39)$ \\
\hline $\mathrm{O}(119)$ & $\operatorname{Re}(1)$ & $\mathrm{O}(11)$ & $C(11)$ & $-57.24(0.40)$ \\
\hline $\mathrm{N}(18)$ & $\operatorname{Re}(1)$ & $\mathrm{O}(11)$ & $\mathrm{C}(11)$ & $31.32(0.41)$ \\
\hline $\mathrm{N}(112)$ & $\operatorname{Re}(1)$ & $\mathrm{O}(11)$ & $C(11)$ & $-41.78(0.86)$ \\
\hline $\mathrm{N}(120)$ & $\operatorname{Re}(1)$ & $\mathrm{O}(11)$ & $\mathrm{C}(11)$ & $122.66(0.43)$ \\
\hline $\mathrm{P}(130)$ & $\operatorname{Re}(1)$ & $\mathrm{O}(119)$ & $C(119)$ & $-69.11(0.50)$ \\
\hline $\mathrm{O}(11)$ & $\operatorname{Re}(1)$ & $\mathrm{O}(119)$ & $C(119)$ & $-155.23(0.52)$ \\
\hline $\mathrm{N}(18)$ & $\operatorname{Re}(1)$ & $\mathrm{O}(119)$ & $C(119)$ & $115.32(0.52)$ \\
\hline $\mathrm{N}(112)$ & $\operatorname{Re}(1)$ & $\mathrm{O}(119)$ & $\mathrm{C}(119)$ & $29.39(0.52)$ \\
\hline $\mathrm{N}(120)$ & $\operatorname{Re}(1)$ & $\mathrm{O}(119)$ & $C(119)$ & $29.87(11.80)$ \\
\hline $\mathrm{P}(130)$ & $\operatorname{Re}(1)$ & $\mathrm{N}(18)$ & $C(17)$ & $23.33(1.56)$ \\
\hline $\mathrm{P}(130)$ & $\operatorname{Re}(1)$ & $\mathrm{N}(18)$ & $C(19)$ & $-164.97(0.94)$ \\
\hline $\mathrm{O}(11)$ & $\operatorname{Re}(1)$ & $\mathrm{N}(18)$ & $C(17)$ & $-19.77(0.49)$ \\
\hline $\mathrm{O}(11)$ & $\operatorname{Re}(1)$ & $\mathrm{N}(18)$ & $C(19)$ & $151.93(0.47)$ \\
\hline $\mathrm{O}(119)$ & $\operatorname{Re}(1)$ & $\mathrm{N}(18)$ & $C(17)$ & $61.11(0.49)$ \\
\hline $\mathrm{O}(119)$ & $\operatorname{Re}(1)$ & $\mathrm{N}(18)$ & $C(19)$ & $-127.18(0.48)$ \\
\hline $\mathrm{N}(112)$ & $\operatorname{Re}(1)$ & $\mathrm{N}(18)$ & $C(17)$ & $143.52(0.50)$ \\
\hline $\mathrm{N}(112)$ & $\operatorname{Re}(1)$ & $\mathrm{N}(18)$ & $\mathrm{C}(19)$ & $-44.78(0.48)$ \\
\hline $\mathrm{N}(120)$ & $\operatorname{Re}(1)$ & $\mathrm{N}(18)$ & $C(17)$ & $-120.00(0.50)$ \\
\hline $\mathrm{N}(120)$ & $\operatorname{Re}(1)$ & $\mathrm{N}(18)$ & $C(19)$ & $51.70(0.49)$ \\
\hline $\mathrm{P}(130)$ & $\operatorname{Re}(1)$ & $\mathrm{N}(112)$ & $C(111)$ & $-130.52(0.41)$ \\
\hline $\mathrm{P}(130)$ & $\operatorname{Re}(1)$ & $\mathrm{N}(112)$ & $C(113)$ & $66.89(0.61)$ \\
\hline $\mathrm{O}(11)$ & $\operatorname{Re}(1)$ & $\mathrm{N}(112)$ & $\mathrm{C}(111)$ & $129.39(0.61)$ \\
\hline $\mathrm{O}(11)$ & $\operatorname{Re}(1)$ & $\mathrm{N}(112)$ & $C(113)$ & $-33.21(1.10)$ \\
\hline $\mathrm{O}(119)$ & $\operatorname{Re}(1)$ & $\mathrm{N}(112)$ & $C(111)$ & $144.79(0.45)$ \\
\hline
\end{tabular}




\begin{tabular}{|c|c|c|c|c|}
\hline $\mathrm{O}(119)$ & $\operatorname{Re}(1)$ & $\mathrm{N}(112)$ & $C(113)$ & $-17.80(0.60)$ \\
\hline $\mathrm{N}(18)$ & $\operatorname{Re}(1)$ & $\mathrm{N}(112)$ & $C(111)$ & $55.78(0.43)$ \\
\hline $\mathrm{N}(18)$ & $\operatorname{Re}(1)$ & $\mathrm{N}(112)$ & $C(113)$ & $-106.81(0.62)$ \\
\hline $\mathrm{N}(120)$ & $\operatorname{Re}(1)$ & $\mathrm{N}(112)$ & $C(111)$ & $-35.20(0.47)$ \\
\hline $\mathrm{N}(120)$ & $\operatorname{Re}(1)$ & $\mathrm{N}(112)$ & $C(113)$ & $162.21(0.63)$ \\
\hline$P(130)$ & $\operatorname{Re}(1)$ & $\mathrm{N}(120)$ & $C(121)$ & $-177.76(3.45)$ \\
\hline $\mathrm{O}(11)$ & $\operatorname{Re}(1)$ & $\mathrm{N}(120)$ & $C(121)$ & $-91.55(3.47)$ \\
\hline$O(119)$ & $\operatorname{Re}(1)$ & $\mathrm{N}(120)$ & $C(121)$ & $83.34(12.29)$ \\
\hline $\mathrm{N}(18)$ & $\operatorname{Re}(1)$ & $\mathrm{N}(120)$ & $C(121)$ & $-2.08(3.47)$ \\
\hline $\mathrm{N}(112)$ & $\operatorname{Re}(1)$ & $\mathrm{N}(120)$ & $C(121)$ & $83.81(3.47)$ \\
\hline $\mathrm{O}(21)$ & $\operatorname{Re}(2)$ & $\mathrm{P}(230)$ & $C(231)$ & $-93.53(0.20)$ \\
\hline $\mathrm{O}(21)$ & $\operatorname{Re}(2)$ & $\mathrm{P}(230)$ & $C(237)$ & $145.91(0.19)$ \\
\hline $\mathrm{O}(21)$ & $\operatorname{Re}(2)$ & $\mathrm{P}(230)$ & $C(243)$ & $23.10(0.22)$ \\
\hline $\mathrm{O}(219)$ & $\operatorname{Re}(2)$ & $\mathrm{P}(230)$ & $C(231)$ & $-174.51(0.21)$ \\
\hline $\mathrm{O}(219)$ & $\operatorname{Re}(2)$ & $\mathrm{P}(230)$ & $C(237)$ & $64.93(0.19)$ \\
\hline $\mathrm{O}(219)$ & $\operatorname{Re}(2)$ & $\mathrm{P}(230)$ & $C(243)$ & $-57.88(0.22)$ \\
\hline $\mathrm{N}(28)$ & $\operatorname{Re}(2)$ & $\mathrm{P}(230)$ & $C(231)$ & $-128.07(1.23)$ \\
\hline $\mathrm{N}(28)$ & $\operatorname{Re}(2)$ & $\mathrm{P}(230)$ & $C(237)$ & $111.36(1.23)$ \\
\hline $\mathrm{N}(28)$ & $\operatorname{Re}(2)$ & $\mathrm{P}(230)$ & $C(243)$ & $-11.44(1.26)$ \\
\hline $\mathrm{N}(212)$ & $\operatorname{Re}(2)$ & $\mathrm{P}(230)$ & $C(231)$ & $104.20(0.22)$ \\
\hline $\mathrm{N}(212)$ & $\operatorname{Re}(2)$ & $\mathrm{P}(230)$ & $C(237)$ & $-16.36(0.21)$ \\
\hline $\mathrm{N}(212)$ & $\operatorname{Re}(2)$ & $\mathrm{P}(230)$ & $C(243)$ & $-139.16(0.23)$ \\
\hline $\mathrm{N}(220)$ & $\operatorname{Re}(2)$ & $\mathrm{P}(230)$ & $C(231)$ & $5.98(0.24)$ \\
\hline $\mathrm{N}(220)$ & $\operatorname{Re}(2)$ & $\mathrm{P}(230)$ & $C(237)$ & $-114.59(0.23)$ \\
\hline $\mathrm{N}(220)$ & $\operatorname{Re}(2)$ & $\mathrm{P}(230)$ & $C(243)$ & $122.61(0.26)$ \\
\hline $\mathrm{P}(230)$ & $\operatorname{Re}(2)$ & $\mathrm{O}(21)$ & $C(21)$ & $-145.68(0.40)$ \\
\hline $\mathrm{O}(219)$ & $\operatorname{Re}(2)$ & $\mathrm{O}(21)$ & $\mathrm{C}(21)$ & $-58.84(0.40)$ \\
\hline $\mathrm{N}(28)$ & $\operatorname{Re}(2)$ & $\mathrm{O}(21)$ & $\mathrm{C}(21)$ & $30.30(0.42)$ \\
\hline$N(212)$ & $\operatorname{Re}(2)$ & $\mathrm{O}(21)$ & $\mathrm{C}(21)$ & $-41.73(0.84)$ \\
\hline $\mathrm{N}(220)$ & $\operatorname{Re}(2)$ & $\mathrm{O}(21)$ & $C(21)$ & $121.32(0.43)$ \\
\hline $\mathrm{P}(230)$ & $\operatorname{Re}(2)$ & $\mathrm{O}(219)$ & $C(219)$ & $-67.47(0.48)$ \\
\hline $\mathrm{O}(21)$ & $\operatorname{Re}(2)$ & $\mathrm{O}(219)$ & $C(219)$ & $-153.47(0.51)$ \\
\hline $\mathrm{N}(28)$ & $\operatorname{Re}(2)$ & $\mathrm{O}(219)$ & $C(219)$ & $117.67(0.51)$ \\
\hline $\mathrm{N}(212)$ & $\operatorname{Re}(2)$ & $\mathrm{O}(219)$ & $C(219)$ & $31.82(0.50)$ \\
\hline $\mathrm{N}(220)$ & $\operatorname{Re}(2)$ & $\mathrm{O}(219)$ & $C(219)$ & $7.78(26.05)$ \\
\hline $\mathrm{P}(230)$ & $\operatorname{Re}(2)$ & $\mathrm{N}(28)$ & $C(27)$ & $17.09(1.62)$ \\
\hline $\mathrm{P}(230)$ & $\operatorname{Re}(2)$ & $\mathrm{N}(28)$ & $\mathrm{C}(29)$ & $-172.13(0.96)$ \\
\hline $\mathrm{O}(21)$ & $\operatorname{Re}(2)$ & $\mathrm{N}(28)$ & $\mathrm{C}(27)$ & $-17.33(0.51)$ \\
\hline $\mathrm{O}(21)$ & $\operatorname{Re}(2)$ & $\mathrm{N}(28)$ & C(29) & $153.44(0.47)$ \\
\hline $\mathrm{O}(219)$ & $\operatorname{Re}(2)$ & $\mathrm{N}(28)$ & $C(27)$ & $63.41(0.51)$ \\
\hline $\mathrm{O}(219)$ & $\operatorname{Re}(2)$ & $\mathrm{N}(28)$ & $C(29)$ & $-125.82(0.47)$ \\
\hline $\mathrm{N}(212)$ & $\operatorname{Re}(2)$ & $\mathrm{N}(28)$ & $\mathrm{C}(27)$ & $145.45(0.53)$ \\
\hline $\mathrm{N}(212)$ & $\operatorname{Re}(2)$ & $\mathrm{N}(28)$ & C(29) & $-43.78(0.47)$ \\
\hline $\mathrm{N}(220)$ & $\operatorname{Re}(2)$ & $\mathrm{N}(28)$ & $C(27)$ & $-117.07(0.53)$ \\
\hline $\mathrm{N}(220)$ & $\operatorname{Re}(2)$ & $\mathrm{N}(28)$ & $C(29)$ & $53.70(0.49)$ \\
\hline $\mathrm{P}(230)$ & $\operatorname{Re}(2)$ & $\mathrm{N}(212)$ & $\mathrm{C}(211)$ & $-131.44(0.42)$ \\
\hline $\mathrm{P}(230)$ & $\operatorname{Re}(2)$ & $\mathrm{N}(212)$ & $C(213)$ & $64.43(0.61)$ \\
\hline $\mathrm{O}(21)$ & $\operatorname{Re}(2)$ & $\mathrm{N}(212)$ & $C(211)$ & $126.67(0.61)$ \\
\hline $\mathrm{O}(21)$ & $\operatorname{Re}(2)$ & $\mathrm{N}(212)$ & $C(213)$ & $-37.46(1.07)$ \\
\hline $\mathrm{O}(219)$ & $\operatorname{Re}(2)$ & $\mathrm{N}(212)$ & $C(211)$ & $143.72(0.45)$ \\
\hline $\mathrm{O}(219)$ & $\operatorname{Re}(2)$ & $\mathrm{N}(212)$ & $C(213)$ & $-20.41(0.60)$ \\
\hline $\mathrm{N}(28)$ & $\operatorname{Re}(2)$ & $\mathrm{N}(212)$ & $C(211)$ & $54.18(0.45)$ \\
\hline $\mathrm{N}(28)$ & $\operatorname{Re}(2)$ & $\mathrm{N}(212)$ & $C(213)$ & $-109.94(0.63)$ \\
\hline $\mathrm{N}(220)$ & $\operatorname{Re}(2)$ & $\mathrm{N}(212)$ & $C(211)$ & $-36.49(0.48)$ \\
\hline $\mathrm{N}(220)$ & $\operatorname{Re}(2)$ & $\mathrm{N}(212)$ & $C(213)$ & $159.39(0.62)$ \\
\hline $\mathrm{P}(230)$ & $\operatorname{Re}(2)$ & $\mathrm{N}(220)$ & $C(221)$ & $-169.25(6.37)$ \\
\hline
\end{tabular}




\begin{tabular}{|c|c|c|c|c|}
\hline $\mathrm{O}(21)$ & $\operatorname{Re}(2)$ & $\mathrm{N}(220)$ & $C(221)$ & $-83.25(6.38)$ \\
\hline $\mathrm{O}(219)$ & $\operatorname{Re}(2)$ & $\mathrm{N}(220)$ & $C(221)$ & $115.53(24.39)$ \\
\hline $\mathrm{N}(28)$ & $\operatorname{Re}(2)$ & $\mathrm{N}(220)$ & $C(221)$ & $5.65(6.38)$ \\
\hline $\mathrm{N}(212)$ & $\operatorname{Re}(2)$ & $\mathrm{N}(220)$ & $C(221)$ & 91.51 ( 6.38) \\
\hline $\operatorname{Re}(1)$ & $\mathrm{P}(130)$ & $C(131)$ & $C(132)$ & $-120.43(0.45)$ \\
\hline $\operatorname{Re}(1)$ & $\mathrm{P}(130)$ & $\mathrm{C}(131)$ & $C(136)$ & $58.40(0.46)$ \\
\hline$C(137)$ & $\mathrm{P}(130)$ & $\mathrm{C}(131)$ & $C(132)$ & $114.34(0.48)$ \\
\hline$C(137)$ & $\mathrm{P}(130)$ & $\mathrm{C}(131)$ & $C(136)$ & $-66.84(0.50)$ \\
\hline$C(143)$ & $\mathrm{P}(130)$ & $\mathrm{C}(131)$ & $C(132)$ & $7.02(0.54)$ \\
\hline$C(143)$ & $\mathrm{P}(130)$ & $\mathrm{C}(131)$ & $C(136)$ & $-174.15(0.44)$ \\
\hline $\operatorname{Re}(1)$ & $\mathrm{P}(130)$ & $\mathrm{C}(137)$ & $C(138)$ & $-139.75(0.46)$ \\
\hline $\operatorname{Re}(1)$ & $\mathrm{P}(130)$ & $\mathrm{C}(137)$ & $C(142)$ & $44.83(0.47)$ \\
\hline$C(131)$ & $\mathrm{P}(130)$ & $C(137)$ & $C(138)$ & $-13.65(0.56)$ \\
\hline$C(131)$ & $\mathrm{P}(130)$ & $\mathrm{C}(137)$ & $C(142)$ & $170.93(0.44)$ \\
\hline$C(143)$ & $\mathrm{P}(130)$ & $\mathrm{C}(137)$ & $C(138)$ & $95.50(0.52)$ \\
\hline$C(143)$ & $\mathrm{P}(130)$ & $\mathrm{C}(137)$ & $C(142)$ & $-79.92(0.49)$ \\
\hline $\operatorname{Re}(1)$ & $\mathrm{P}(130)$ & $C(143)$ & $C(144)$ & $-146.04(0.44)$ \\
\hline $\operatorname{Re}(1)$ & $\mathrm{P}(130)$ & $C(143)$ & $C(148)$ & $36.35(0.52)$ \\
\hline$C(131)$ & $\mathrm{P}(130)$ & $C(143)$ & $C(144)$ & $86.62(0.52)$ \\
\hline$C(131)$ & $\mathrm{P}(130)$ & $C(143)$ & $C(148)$ & $-90.99(0.50)$ \\
\hline$C(137)$ & $\mathrm{P}(130)$ & $C(143)$ & $C(144)$ & $-22.32(0.55)$ \\
\hline$C(137)$ & $\mathrm{P}(130)$ & $C(143)$ & $C(148)$ & $160.07(0.47)$ \\
\hline $\operatorname{Re}(2)$ & $\mathrm{P}(230)$ & $C(231)$ & $C(232)$ & 48.73 ( 0.49) \\
\hline $\operatorname{Re}(2)$ & $\mathrm{P}(230)$ & $\mathrm{C}(231)$ & $C(236)$ & $-137.13(0.48)$ \\
\hline$C(237)$ & $\mathrm{P}(230)$ & $C(231)$ & $C(232)$ & $175.66(0.46)$ \\
\hline$C(237)$ & $\mathrm{P}(230)$ & $C(231)$ & $C(236)$ & $-10.21(0.58)$ \\
\hline$C(243)$ & $\mathrm{P}(230)$ & $C(231)$ & $C(232)$ & $-75.53(0.51)$ \\
\hline$C(243)$ & $\mathrm{P}(230)$ & $C(231)$ & $C(236)$ & $98.61(0.54)$ \\
\hline $\operatorname{Re}(2)$ & $\mathrm{P}(230)$ & $C(237)$ & $C(238)$ & $-121.59(0.46)$ \\
\hline $\operatorname{Re}(2)$ & $\mathrm{P}(230)$ & $\mathrm{C}(237)$ & $C(242)$ & $56.31(0.46)$ \\
\hline$C(231)$ & $\mathrm{P}(230)$ & $\mathrm{C}(237)$ & $C(238)$ & $114.05(0.49)$ \\
\hline$C(231)$ & $\mathrm{P}(230)$ & $C(237)$ & $C(242)$ & $-68.05(0.49)$ \\
\hline$C(243)$ & $\mathrm{P}(230)$ & $\mathrm{C}(237)$ & $C(238)$ & $6.78(0.54)$ \\
\hline$C(243)$ & $\mathrm{P}(230)$ & $C(237)$ & $C(242)$ & $-175.32(0.43)$ \\
\hline $\operatorname{Re}(2)$ & $\mathrm{P}(230)$ & $C(243)$ & $C(244)$ & $-146.02(0.42)$ \\
\hline $\operatorname{Re}(2)$ & $\mathrm{P}(230)$ & $C(243)$ & $C(248)$ & $37.50(0.52)$ \\
\hline$C(231)$ & $\mathrm{P}(230)$ & $C(243)$ & $C(244)$ & $-24.00(0.54)$ \\
\hline$C(231)$ & $\mathrm{P}(230)$ & $C(243)$ & $C(248)$ & $159.52(0.47)$ \\
\hline$C(237)$ & $\mathrm{P}(230)$ & $C(243)$ & $C(244)$ & $85.14(0.50)$ \\
\hline$C(237)$ & $\mathrm{P}(230)$ & $C(243)$ & $C(248)$ & $-91.34(0.50)$ \\
\hline $\operatorname{Re}(1)$ & $\mathrm{O}(11)$ & $\mathrm{C}(11)$ & $\mathrm{C}(12)$ & $153.49(0.38)$ \\
\hline $\operatorname{Re}(1)$ & $\mathrm{O}(11)$ & $\mathrm{C}(11)$ & $C(16)$ & $-27.78(0.71)$ \\
\hline $\operatorname{Re}(2)$ & $\mathrm{O}(21)$ & $\mathrm{C}(21)$ & $C(22)$ & $153.16(0.39)$ \\
\hline $\operatorname{Re}(2)$ & $\mathrm{O}(21)$ & $\mathrm{C}(21)$ & $C(26)$ & $-27.33(0.71)$ \\
\hline $\operatorname{Re}(1)$ & $\mathrm{O}(119)$ & $\mathrm{C}(119)$ & $C(114)$ & $-21.79(0.86)$ \\
\hline $\operatorname{Re}(1)$ & $\mathrm{O}(119)$ & $\mathrm{C}(119)$ & $C(118)$ & $159.34(0.49)$ \\
\hline $\operatorname{Re}(2)$ & $\mathrm{O}(219)$ & $\mathrm{C}(219)$ & $C(214)$ & $-24.51(0.86)$ \\
\hline $\operatorname{Re}(2)$ & $\mathrm{O}(219)$ & $\mathrm{C}(219)$ & $C(218)$ & $158.59(0.48)$ \\
\hline $\operatorname{Re}(1)$ & $\mathrm{N}(18)$ & $\mathrm{C}(17)$ & $C(16)$ & $4.00(0.88)$ \\
\hline$C(19)$ & $\mathrm{N}(18)$ & $\mathrm{C}(17)$ & $C(16)$ & $-168.02(0.60)$ \\
\hline $\operatorname{Re}(1)$ & $\mathrm{N}(18)$ & $\mathrm{C}(19)$ & $C(110)$ & $50.50(0.68)$ \\
\hline $\mathrm{C}(17)$ & $\mathrm{N}(18)$ & $C(19)$ & $C(110)$ & $-137.40(0.60)$ \\
\hline $\operatorname{Re}(2)$ & $\mathrm{N}(28)$ & $\mathrm{C}(27)$ & $C(26)$ & $0.16(0.89)$ \\
\hline$C(29)$ & $\mathrm{N}(28)$ & $\mathrm{C}(27)$ & $C(26)$ & $-170.66(0.59)$ \\
\hline $\operatorname{Re}(2)$ & $\mathrm{N}(28)$ & $C(29)$ & $C(210)$ & $52.73(0.74)$ \\
\hline$C(27)$ & $\mathrm{N}(28)$ & $\mathrm{C}(29)$ & $C(210)$ & $-136.12(0.67)$ \\
\hline
\end{tabular}




\begin{tabular}{|c|c|c|c|c|}
\hline $\operatorname{Re}(1)$ & $\mathrm{N}(112)$ & $C(111)$ & $C(110)$ & $-75.92(0.58)$ \\
\hline$C(113)$ & $\mathrm{N}(112)$ & $C(111)$ & $C(110)$ & $88.98(0.70)$ \\
\hline $\operatorname{Re}(1)$ & $\mathrm{N}(112)$ & $C(113)$ & $C(114)$ & $0.43(1.10)$ \\
\hline $\mathrm{C}(111)$ & $\mathrm{N}(112)$ & $\mathrm{C}(113)$ & $C(114)$ & $-161.99(0.67)$ \\
\hline $\operatorname{Re}(1)$ & $\mathrm{N}(120)$ & $C(121)$ & $C(122)$ & $-126.00(3.32)$ \\
\hline $\operatorname{Re}(1)$ & $\mathrm{N}(120)$ & $\mathrm{C}(121)$ & $C(126)$ & $54.03(3.65)$ \\
\hline $\operatorname{Re}(2)$ & $\mathrm{N}(212)$ & $C(211)$ & $C(210)$ & $-74.61(0.70)$ \\
\hline$C(213)$ & $\mathrm{N}(212)$ & $\mathrm{C}(211)$ & $C(210)$ & $91.74(0.78)$ \\
\hline $\operatorname{Re}(2)$ & $\mathrm{N}(212)$ & $C(213)$ & $C(214)$ & $3.30(1.13)$ \\
\hline$C(211)$ & $\mathrm{N}(212)$ & $C(213)$ & $C(214)$ & $-160.91(0.71)$ \\
\hline $\operatorname{Re}(2)$ & $\mathrm{N}(220)$ & $C(221)$ & $C(222)$ & $49.64(6.59)$ \\
\hline $\operatorname{Re}(2)$ & $\mathrm{N}(220)$ & $\mathrm{C}(221)$ & $C(226)$ & $-131.25(6.17)$ \\
\hline $\mathrm{O}(11)$ & $\mathrm{C}(11)$ & $\mathrm{C}(12)$ & $\mathrm{C}(13)$ & $179.00(0.54)$ \\
\hline$C(16)$ & $\mathrm{C}(11)$ & $\mathrm{C}(12)$ & $\mathrm{C}(13)$ & $0.20(0.85)$ \\
\hline $\mathrm{O}(11)$ & $\mathrm{C}(11)$ & $C(16)$ & $C(15)$ & $-177.35(0.55)$ \\
\hline $\mathrm{O}(11)$ & $C(11)$ & $C(16)$ & $\mathrm{C}(17)$ & $1.93(0.89)$ \\
\hline$C(12)$ & $\mathrm{C}(11)$ & $C(16)$ & $C(15)$ & $1.37(0.82)$ \\
\hline$C(12)$ & $C(11)$ & $C(16)$ & $C(17)$ & $-179.35(0.54)$ \\
\hline $\mathrm{C}(11)$ & $\mathrm{C}(12)$ & $\mathrm{C}(13)$ & $\mathrm{C}(14)$ & $-1.67(0.98)$ \\
\hline$C(12)$ & $\mathrm{C}(13)$ & $\mathrm{C}(14)$ & $C(15)$ & $1.49(1.06)$ \\
\hline$C(13)$ & $C(14)$ & $C(15)$ & $C(16)$ & $0.13(1.07)$ \\
\hline$C(14)$ & $C(15)$ & $C(16)$ & $\mathrm{C}(11)$ & $-1.56(0.98)$ \\
\hline$C(14)$ & $\mathrm{C}(15)$ & $C(16)$ & $\mathrm{C}(17)$ & $179.11(0.64)$ \\
\hline $\mathrm{C}(11)$ & $C(16)$ & $C(17)$ & $\mathrm{N}(18)$ & $10.86(1.00)$ \\
\hline$C(15)$ & $C(16)$ & $\mathrm{C}(17)$ & $\mathrm{N}(18)$ & $-169.86(0.64)$ \\
\hline $\mathrm{N}(18)$ & $\mathrm{C}(19)$ & $C(110)$ & $C(111)$ & $-58.15(0.74)$ \\
\hline $\mathrm{O}(21)$ & $\mathrm{C}(21)$ & $\mathrm{C}(22)$ & $\mathrm{C}(23)$ & $177.64(0.54)$ \\
\hline$C(26)$ & $C(21)$ & $C(22)$ & $C(23)$ & $-1.90(0.85)$ \\
\hline $\mathrm{O}(21)$ & $\mathrm{C}(21)$ & $C(26)$ & $C(25)$ & $-175.32(0.55)$ \\
\hline $\mathrm{O}(21)$ & $C(21)$ & $C(26)$ & $C(27)$ & $0.61(0.89)$ \\
\hline$C(22)$ & $\mathrm{C}(21)$ & $C(26)$ & $C(25)$ & $4.18(0.83)$ \\
\hline$C(22)$ & $\mathrm{C}(21)$ & $C(26)$ & $C(27)$ & $-179.89(0.53)$ \\
\hline$C(21)$ & $C(22)$ & $C(23)$ & $C(24)$ & $-0.39(0.98)$ \\
\hline$C(22)$ & $\mathrm{C}(23)$ & $\mathrm{C}(24)$ & $C(25)$ & $0.31(1.07)$ \\
\hline$C(23)$ & $\mathrm{C}(24)$ & $C(25)$ & $C(26)$ & $2.11(1.08)$ \\
\hline$C(24)$ & $C(25)$ & $C(26)$ & $\mathrm{C}(21)$ & $-4.35(0.97)$ \\
\hline$C(24)$ & $C(25)$ & $C(26)$ & $C(27)$ & $179.41(0.62)$ \\
\hline$C(21)$ & $C(26)$ & $C(27)$ & $\mathrm{N}(28)$ & $13.99(0.99)$ \\
\hline$C(25)$ & $C(26)$ & $\mathrm{C}(27)$ & $\mathrm{N}(28)$ & $-170.02(0.64)$ \\
\hline $\mathrm{N}(28)$ & $C(29)$ & $C(210)$ & $C(211)$ & $-60.73(0.85)$ \\
\hline C(19) & $C(110)$ & $C(111)$ & $\mathrm{N}(112)$ & $70.72(0.71)$ \\
\hline $\mathrm{N}(112)$ & $C(113)$ & $\mathrm{C}(114)$ & $C(115)$ & $-170.67(0.70)$ \\
\hline $\mathrm{N}(112)$ & $C(113)$ & $C(114)$ & $C(119)$ & $17.97(1.08)$ \\
\hline$C(113)$ & $C(114)$ & $C(115)$ & $C(116)$ & $-172.12(0.70)$ \\
\hline$C(119)$ & $C(114)$ & $C(115)$ & $C(116)$ & $-0.75(1.04)$ \\
\hline$C(113)$ & $C(114)$ & $\mathrm{C}(119)$ & $\mathrm{O}(119)$ & $-8.19(0.92)$ \\
\hline$C(113)$ & $C(114)$ & $\mathrm{C}(119)$ & $C(118)$ & $170.70(0.64)$ \\
\hline$C(115)$ & $C(114)$ & $C(119)$ & $\mathrm{O}(119)$ & $-179.50(0.58)$ \\
\hline$C(115)$ & $C(114)$ & $\mathrm{C}(119)$ & $C(118)$ & $-0.60(0.94)$ \\
\hline$C(114)$ & $C(115)$ & $C(116)$ & $C(117)$ & $1.49(1.16)$ \\
\hline$C(115)$ & $C(116)$ & $C(117)$ & $C(118)$ & $-0.87(1.20)$ \\
\hline$C(116)$ & $C(117)$ & $\mathrm{C}(118)$ & $C(119)$ & $-0.51(1.18)$ \\
\hline$C(117)$ & $\mathrm{C}(118)$ & $\mathrm{C}(119)$ & $\mathrm{O}(119)$ & $-179.87(0.66)$ \\
\hline$C(117)$ & $\mathrm{C}(118)$ & $\mathrm{C}(119)$ & $C(114)$ & $1.22(1.05)$ \\
\hline $\mathrm{N}(120)$ & $C(121)$ & $C(122)$ & $C(123)$ & $175.84(0.64)$ \\
\hline $\mathrm{N}(120)$ & $C(121)$ & $C(122)$ & $C(127)$ & $-6.84(1.01)$ \\
\hline
\end{tabular}




\begin{tabular}{|c|c|c|c|c|}
\hline$C(126)$ & $C(121)$ & $C(122)$ & $C(123)$ & $-4.20(0.99)$ \\
\hline$C(126)$ & $C(121)$ & $C(122)$ & $C(127)$ & $173.13(0.62)$ \\
\hline $\mathrm{N}(120)$ & $C(121)$ & $C(126)$ & $C(125)$ & $-176.29(0.58)$ \\
\hline $\mathrm{N}(120)$ & $C(121)$ & $C(126)$ & $C(129)$ & $3.76(0.87)$ \\
\hline$C(122)$ & $\mathrm{C}(121)$ & $C(126)$ & $C(125)$ & $3.74(0.89)$ \\
\hline$C(122)$ & $C(121)$ & $C(126)$ & $C(129)$ & $-176.20(0.62)$ \\
\hline$C(121)$ & $\mathrm{C}(122)$ & $\mathrm{C}(123)$ & $C(124)$ & $1.17(1.14)$ \\
\hline C(127) & $\mathrm{C}(122)$ & $\mathrm{C}(123)$ & $C(124)$ & $-176.17(0.72)$ \\
\hline$C(122)$ & $C(123)$ & $\mathrm{C}(124)$ & $C(125)$ & $2.18(1.17)$ \\
\hline$C(122)$ & $C(123)$ & $C(124)$ & $C(128)$ & $-179.55(0.74)$ \\
\hline$C(123)$ & $C(124)$ & $C(125)$ & $C(126)$ & $-2.65(1.11)$ \\
\hline$C(128)$ & $C(124)$ & $C(125)$ & $C(126)$ & $179.05(0.67)$ \\
\hline C(124) & $C(125)$ & $C(126)$ & $C(121)$ & $-0.30(0.98)$ \\
\hline $\mathrm{C}(124)$ & $C(125)$ & $C(126)$ & $C(129)$ & $179.65(0.67)$ \\
\hline $\mathrm{P}(130)$ & $C(131)$ & $C(132)$ & $C(133)$ & $178.14(0.47)$ \\
\hline$C(136)$ & $\mathrm{C}(131)$ & $C(132)$ & $C(133)$ & $-0.69(0.84)$ \\
\hline $\mathrm{P}(130)$ & $C(131)$ & $C(136)$ & $C(135)$ & $-178.47(0.45)$ \\
\hline$C(132)$ & $C(131)$ & $C(136)$ & $C(135)$ & $0.41(0.83)$ \\
\hline$C(131)$ & $C(132)$ & $\mathrm{C}(133)$ & $C(134)$ & $0.91(0.96)$ \\
\hline C(132) & $C(133)$ & $C(134)$ & $C(135)$ & $-0.84(1.06)$ \\
\hline$C(133)$ & $C(134)$ & $C(135)$ & $C(136)$ & $0.56(1.02)$ \\
\hline$C(134)$ & $C(135)$ & $C(136)$ & $C(131)$ & $-0.34(0.90)$ \\
\hline $\mathrm{P}(130)$ & $\mathrm{C}(137)$ & $\mathrm{C}(138)$ & $\mathrm{C}(139)$ & $-177.01(0.51)$ \\
\hline$C(142)$ & $\mathrm{C}(137)$ & $\mathrm{C}(138)$ & $C(139)$ & $-1.63(0.92)$ \\
\hline $\mathrm{P}(130)$ & $C(137)$ & $C(142)$ & $C(141)$ & $176.77(0.48)$ \\
\hline$C(138)$ & $C(137)$ & $C(142)$ & $C(141)$ & $1.19(0.87)$ \\
\hline$C(137)$ & $\mathrm{C}(138)$ & $\mathrm{C}(139)$ & $C(140)$ & $1.81(1.06)$ \\
\hline$C(138)$ & $C(139)$ & $C(140)$ & $C(141)$ & $-1.53(1.14)$ \\
\hline$C(139)$ & $C(140)$ & $C(141)$ & $C(142)$ & $1.06(1.08)$ \\
\hline$C(140)$ & $C(141)$ & $C(142)$ & $C(137)$ & $-0.89(0.96)$ \\
\hline $\mathrm{P}(130)$ & $\mathrm{C}(143)$ & $\mathrm{C}(144)$ & $C(145)$ & $-176.46(0.51)$ \\
\hline$C(148)$ & $C(143)$ & $C(144)$ & $C(145)$ & $1.17(0.93)$ \\
\hline$P(130)$ & $C(143)$ & $C(148)$ & $C(147)$ & $175.43(0.49)$ \\
\hline$C(144)$ & $C(143)$ & $C(148)$ & $C(147)$ & $-2.19(0.88)$ \\
\hline$C(143)$ & $C(144)$ & $C(145)$ & $C(146)$ & $0.07(1.14)$ \\
\hline$C(144)$ & $C(145)$ & $C(146)$ & $C(147)$ & $-0.25(1.14)$ \\
\hline$C(145)$ & $C(146)$ & $C(147)$ & $C(148)$ & $-0.81(1.14)$ \\
\hline$C(146)$ & $C(147)$ & $\mathrm{C}(148)$ & $C(143)$ & $2.03(1.02)$ \\
\hline$C(29)$ & $\mathrm{C}(210)$ & $\mathrm{C}(211)$ & $N(212)$ & $70.52(0.85)$ \\
\hline $\mathrm{N}(212)$ & $C(213)$ & $C(214)$ & $C(215)$ & $-171.10(0.75)$ \\
\hline $\mathrm{N}(212)$ & $C(213)$ & $C(214)$ & $C(219)$ & $16.01(1.17)$ \\
\hline$C(213)$ & $C(214)$ & $C(215)$ & $C(216)$ & $-172.62(0.76)$ \\
\hline$C(219)$ & $C(214)$ & $C(215)$ & $C(216)$ & $0.35(1.15)$ \\
\hline$C(213)$ & $C(214)$ & $C(219)$ & $\mathrm{O}(219)$ & $-6.11(0.99)$ \\
\hline$C(213)$ & $C(214)$ & $C(219)$ & $C(218)$ & $170.75(0.67)$ \\
\hline$C(215)$ & $C(214)$ & $C(219)$ & $\mathrm{O}(219)$ & $-179.18(0.62)$ \\
\hline$C(215)$ & $C(214)$ & $C(219)$ & $C(218)$ & $-2.32(1.03)$ \\
\hline$C(214)$ & $C(215)$ & $C(216)$ & $C(217)$ & $1.18(1.23)$ \\
\hline$C(215)$ & $C(216)$ & $C(217)$ & $C(218)$ & $-0.75(1.21)$ \\
\hline$C(216)$ & $C(217)$ & $\mathrm{C}(218)$ & $C(219)$ & $-1.23(1.15)$ \\
\hline$C(217)$ & $\mathrm{C}(218)$ & $C(219)$ & $O(219)$ & $179.73(0.63)$ \\
\hline$C(217)$ & $C(218)$ & $C(219)$ & $C(214)$ & $2.75(1.07)$ \\
\hline $\mathrm{N}(220)$ & $C(221)$ & $C(222)$ & $C(223)$ & $-178.49(0.59)$ \\
\hline $\mathrm{N}(220)$ & $\mathrm{C}(221)$ & $C(222)$ & $C(227)$ & $0.61(0.87)$ \\
\hline$C(226)$ & $C(221)$ & $C(222)$ & $C(223)$ & $2.39(0.92)$ \\
\hline$C(226)$ & $\mathrm{C}(221)$ & $\mathrm{C}(222)$ & $C(227)$ & $-178.51(0.63)$ \\
\hline
\end{tabular}




\begin{tabular}{|c|c|c|c|c|}
\hline $\mathrm{N}(220)$ & $C(221)$ & $C(226)$ & $C(225)$ & $179.01(0.65)$ \\
\hline $\mathrm{N}(220)$ & $C(221)$ & $C(226)$ & $C(229)$ & $-3.65(1.05)$ \\
\hline$C(222)$ & $C(221)$ & $C(226)$ & $C(225)$ & $-1.90(1.00)$ \\
\hline$C(222)$ & $C(221)$ & $C(226)$ & $C(229)$ & $175.44(0.66)$ \\
\hline$C(221)$ & $C(222)$ & $C(223)$ & $C(224)$ & $-0.84(1.02)$ \\
\hline$C(227)$ & $C(222)$ & $C(223)$ & $C(224)$ & $-179.91(0.70)$ \\
\hline$C(222)$ & $C(223)$ & $C(224)$ & $C(225)$ & $-1.21(1.14)$ \\
\hline$C(222)$ & $C(223)$ & $C(224)$ & $\mathrm{C}(228)$ & $179.74(0.70)$ \\
\hline$C(223)$ & $C(224)$ & $C(225)$ & $C(226)$ & $1.71(1.17)$ \\
\hline$C(228)$ & $C(224)$ & $C(225)$ & $C(226)$ & $-179.22(0.73)$ \\
\hline$C(224)$ & $C(225)$ & $C(226)$ & $C(221)$ & $-0.17(1.14)$ \\
\hline$C(224)$ & $C(225)$ & $C(226)$ & $C(229)$ & $-177.56(0.74)$ \\
\hline $\mathrm{P}(230)$ & $C(231)$ & $C(232)$ & $C(233)$ & $175.23(0.54)$ \\
\hline$C(236)$ & $C(231)$ & $C(232)$ & $C(233)$ & $0.84(0.93)$ \\
\hline $\mathrm{P}(230)$ & $C(231)$ & $C(236)$ & $C(235)$ & $-176.56(0.55)$ \\
\hline$C(232)$ & $C(231)$ & $C(236)$ & $C(235)$ & $-2.45(0.96)$ \\
\hline$C(231)$ & $C(232)$ & $C(233)$ & $C(234)$ & $2.31(1.13)$ \\
\hline$C(232)$ & $C(233)$ & $C(234)$ & $C(235)$ & $-3.85(1.29)$ \\
\hline$C(233)$ & $C(234)$ & $C(235)$ & $C(236)$ & $2.22(1.29)$ \\
\hline$C(234)$ & $C(235)$ & $C(236)$ & $C(231)$ & $0.93(1.15)$ \\
\hline $\mathrm{P}(230)$ & $C(237)$ & $C(238)$ & $C(239)$ & $178.76(0.49)$ \\
\hline$C(242)$ & $C(237)$ & $C(238)$ & $C(239)$ & $0.86(0.87)$ \\
\hline $\mathrm{P}(230)$ & $C(237)$ & $C(242)$ & $C(241)$ & $-178.96(0.49)$ \\
\hline$C(238)$ & $C(237)$ & $C(242)$ & $C(241)$ & $-1.00(0.86)$ \\
\hline$C(237)$ & $C(238)$ & $C(239)$ & $C(240)$ & $0.09(1.04)$ \\
\hline$C(238)$ & $C(239)$ & $C(240)$ & $C(241)$ & $-0.94(1.11)$ \\
\hline$C(239)$ & $C(240)$ & $C(241)$ & $C(242)$ & $0.80(1.08)$ \\
\hline$C(240)$ & $C(241)$ & $C(242)$ & $C(237)$ & $0.16(0.98)$ \\
\hline $\mathrm{P}(230)$ & $C(243)$ & $C(244)$ & $C(245)$ & $-177.23(0.51)$ \\
\hline$C(248)$ & $C(243)$ & $C(244)$ & $C(245)$ & $-0.71(0.89)$ \\
\hline $\mathrm{P}(230)$ & $C(243)$ & $C(248)$ & $C(247)$ & $176.13(0.49)$ \\
\hline$C(244)$ & $C(243)$ & $C(248)$ & $C(247)$ & $-0.39(0.87)$ \\
\hline$C(243)$ & $C(244)$ & $C(245)$ & $C(246)$ & $1.49(1.03)$ \\
\hline$C(244)$ & $C(245)$ & $C(246)$ & $C(247)$ & $-1.14(1.12)$ \\
\hline$C(245)$ & $C(246)$ & $C(247)$ & $C(248)$ & $0.05(1.12)$ \\
\hline$C(246)$ & $C(247)$ & $\mathrm{C}(248)$ & $C(243)$ & $0.73(1.00)$ \\
\hline$C(316)$ & $\mathrm{C}(311)$ & $C(312)$ & $\mathrm{F}(312)$ & $-178.82(0.51)$ \\
\hline$C(316)$ & $C(311)$ & $C(312)$ & $C(313)$ & $2.15(0.86)$ \\
\hline $\mathrm{B}(3)$ & $\mathrm{C}(311)$ & $C(312)$ & $\mathrm{F}(312)$ & $-6.97(0.78)$ \\
\hline $\mathrm{B}(3)$ & $\mathrm{C}(311)$ & $C(312)$ & $C(313)$ & $173.99(0.58)$ \\
\hline$C(312)$ & $C(311)$ & $C(316)$ & $F(316)$ & $178.92(0.51)$ \\
\hline$C(312)$ & $C(311)$ & $C(316)$ & $C(315)$ & $-2.05(0.84)$ \\
\hline B(3) & $\mathrm{C}(311)$ & $C(316)$ & $\mathrm{F}(316)$ & $7.74(0.84)$ \\
\hline $\mathrm{B}(3)$ & $C(311)$ & $C(316)$ & $C(315)$ & $-173.22(0.56)$ \\
\hline$C(312)$ & $C(311)$ & $\mathrm{B}(3)$ & $C(321)$ & $-66.05(0.68)$ \\
\hline$C(312)$ & $\mathrm{C}(311)$ & $\mathrm{B}(3)$ & $C(331)$ & $170.03(0.49)$ \\
\hline$C(312)$ & $C(311)$ & $\mathrm{B}(3)$ & $C(341)$ & $55.13(0.65)$ \\
\hline$C(316)$ & $\mathrm{C}(311)$ & $\mathrm{B}(3)$ & $C(321)$ & $104.43(0.63)$ \\
\hline$C(316)$ & $C(311)$ & $\mathrm{B}(3)$ & $C(331)$ & $-19.49(0.76)$ \\
\hline$C(316)$ & $C(311)$ & $\mathrm{B}(3)$ & $C(341)$ & $-134.40(0.57)$ \\
\hline $\mathrm{F}(312)$ & $C(312)$ & $C(313)$ & $F(313)$ & $-0.90(0.90)$ \\
\hline$F(312)$ & $C(312)$ & $C(313)$ & $C(314)$ & $179.58(0.57)$ \\
\hline$C(311)$ & $\mathrm{C}(312)$ & $C(313)$ & $\mathrm{F}(313)$ & $178.15(0.55)$ \\
\hline$C(311)$ & $C(312)$ & $C(313)$ & $C(314)$ & $-1.38(1.01)$ \\
\hline$F(313)$ & $\mathrm{C}(313)$ & $C(314)$ & $F(314)$ & $1.43(0.95)$ \\
\hline$F(313)$ & $C(313)$ & $C(314)$ & $C(315)$ & $-179.28(0.58)$ \\
\hline
\end{tabular}




\begin{tabular}{|c|c|c|c|c|}
\hline$C(312)$ & $C(313)$ & $C(314)$ & $\mathrm{F}(314)$ & $-179.05(0.59)$ \\
\hline$C(312)$ & $C(313)$ & $C(314)$ & $C(315)$ & $0.25(0.98)$ \\
\hline$F(314)$ & $C(314)$ & $C(315)$ & $F(315)$ & $0.98(0.94)$ \\
\hline$F(314)$ & $C(314)$ & $C(315)$ & $C(316)$ & $179.14(0.58)$ \\
\hline$C(313)$ & $C(314)$ & $C(315)$ & $\mathrm{F}(315)$ & $-178.32(0.58)$ \\
\hline$C(313)$ & $C(314)$ & $C(315)$ & $C(316)$ & $-0.16(0.97)$ \\
\hline$F(315)$ & $C(315)$ & $C(316)$ & $F(316)$ & $-1.58(0.86)$ \\
\hline$F(315)$ & $C(315)$ & $C(316)$ & $\mathrm{C}(311)$ & $179.33(0.54)$ \\
\hline$C(314)$ & $C(315)$ & $C(316)$ & $F(316)$ & $-179.73(0.55)$ \\
\hline$C(314)$ & $C(315)$ & $C(316)$ & $C(311)$ & 1.18 ( 0.99) \\
\hline$C(326)$ & $C(321)$ & $C(322)$ & $F(322)$ & $178.18(0.55)$ \\
\hline$C(326)$ & $C(321)$ & $C(322)$ & $C(323)$ & $0.04(1.03)$ \\
\hline B(3) & $C(321)$ & $C(322)$ & $\mathrm{F}(322)$ & $4.22(1.05)$ \\
\hline B(3) & $C(321)$ & $C(322)$ & $C(323)$ & $-173.92(0.68)$ \\
\hline$C(322)$ & $\mathrm{C}(321)$ & $C(326)$ & $F(326)$ & $-178.19(0.55)$ \\
\hline$C(322)$ & $C(321)$ & $C(326)$ & $C(325)$ & $2.47(0.91)$ \\
\hline $\mathrm{B}(3)$ & $\mathrm{C}(321)$ & $C(326)$ & $F(326)$ & $-3.47(0.83)$ \\
\hline B(3) & $C(321)$ & $C(326)$ & $C(325)$ & $177.19(0.61)$ \\
\hline$C(322)$ & $\mathrm{C}(321)$ & $\mathrm{B}(3)$ & $\mathrm{C}(311)$ & $101.88(0.73)$ \\
\hline$C(322)$ & $C(321)$ & $\mathrm{B}(3)$ & $C(331)$ & $-134.56(0.70)$ \\
\hline$C(322)$ & $C(321)$ & $\mathrm{B}(3)$ & $C(341)$ & $-18.87(0.94)$ \\
\hline$C(326)$ & $C(321)$ & $\mathrm{B}(3)$ & $C(311)$ & $-71.77(0.68)$ \\
\hline$C(326)$ & $\mathrm{C}(321)$ & $\mathrm{B}(3)$ & $C(331)$ & $51.79(0.73)$ \\
\hline$C(326)$ & $C(321)$ & B(3) & $C(341)$ & $167.47(0.53)$ \\
\hline$F(322)$ & $C(322)$ & $C(323)$ & $F(323)$ & $3.27(1.03)$ \\
\hline$F(322)$ & $C(322)$ & $C(323)$ & $C(324)$ & $178.47(0.66)$ \\
\hline$C(321)$ & $C(322)$ & $C(323)$ & $\mathrm{F}(323)$ & $-178.55(0.67)$ \\
\hline$C(321)$ & $C(322)$ & $C(323)$ & $C(324)$ & $-3.35(1.21)$ \\
\hline$F(323)$ & $C(323)$ & $C(324)$ & $F(324)$ & $-3.67(1.14)$ \\
\hline$F(323)$ & $C(323)$ & $C(324)$ & $C(325)$ & 179.29 ( 0.69) \\
\hline$C(322)$ & $C(323)$ & $C(324)$ & $F(324)$ & $-178.82(0.69)$ \\
\hline$C(322)$ & $C(323)$ & $C(324)$ & $C(325)$ & 4.15 ( 1.17$)$ \\
\hline$F(324)$ & $C(324)$ & $C(325)$ & $F(325)$ & $2.73(1.10)$ \\
\hline$F(324)$ & $C(324)$ & $C(325)$ & $C(326)$ & $-178.79(0.65)$ \\
\hline$C(323)$ & $C(324)$ & $C(325)$ & $\mathrm{F}(325)$ & $179.79(0.68)$ \\
\hline$C(323)$ & $C(324)$ & $C(325)$ & $C(326)$ & $-1.73(1.11)$ \\
\hline$F(325)$ & $C(325)$ & $C(326)$ & $F(326)$ & $-2.57(0.92)$ \\
\hline$F(325)$ & $C(325)$ & $C(326)$ & $C(321)$ & $176.79(0.58)$ \\
\hline$C(324)$ & $C(325)$ & $C(326)$ & $F(326)$ & $178.95(0.61)$ \\
\hline C(324) & $C(325)$ & $C(326)$ & $C(321)$ & $-1.69(1.05)$ \\
\hline$C(336)$ & $C(331)$ & $C(332)$ & $F(332)$ & $-176.74(0.46)$ \\
\hline$C(336)$ & $\mathrm{C}(331)$ & $C(332)$ & $C(333)$ & $2.98(0.79)$ \\
\hline B(3) & $C(331)$ & $C(332)$ & $F(332)$ & $-1.91(0.83)$ \\
\hline $\mathrm{B}(3)$ & $C(331)$ & $C(332)$ & $C(333)$ & $177.80(0.54)$ \\
\hline$C(332)$ & $C(331)$ & $C(336)$ & $F(336)$ & $176.58(0.46)$ \\
\hline$C(332)$ & $C(331)$ & $C(336)$ & $C(335)$ & $-3.00(0.83)$ \\
\hline B(3) & $C(331)$ & $C(336)$ & $F(336)$ & $1.45(0.78)$ \\
\hline $\mathrm{B}(3)$ & $C(331)$ & $C(336)$ & $C(335)$ & $-178.13(0.58)$ \\
\hline$C(332)$ & $C(331)$ & $\mathrm{B}(3)$ & $C(311)$ & $134.64(0.55)$ \\
\hline$C(332)$ & $C(331)$ & $\mathrm{B}(3)$ & $C(321)$ & $18.24(0.83)$ \\
\hline$C(332)$ & $C(331)$ & $\mathrm{B}(3)$ & $C(341)$ & $-104.06(0.59)$ \\
\hline$C(336)$ & $C(331)$ & $\mathrm{B}(3)$ & $C(311)$ & $-50.98(0.71)$ \\
\hline$C(336)$ & $C(331)$ & $\mathrm{B}(3)$ & $C(321)$ & $-167.37(0.51)$ \\
\hline$C(336)$ & $C(331)$ & B(3) & $C(341)$ & $70.32(0.63)$ \\
\hline$F(332)$ & $C(332)$ & $C(333)$ & $F(333)$ & $-1.35(0.73)$ \\
\hline$F(332)$ & $C(332)$ & $C(333)$ & $C(334)$ & $178.15(0.46)$ \\
\hline
\end{tabular}




\begin{tabular}{|c|c|c|c|c|}
\hline$C(331)$ & $C(332)$ & $C(333)$ & $F(333)$ & $178.92(0.50)$ \\
\hline$C(331)$ & $C(332)$ & $C(333)$ & $C(334)$ & $-1.58(0.84)$ \\
\hline $\mathrm{F}(333)$ & $C(333)$ & $C(334)$ & $F(334)$ & $-2.95(0.77)$ \\
\hline $\mathrm{F}(333)$ & $C(333)$ & $C(334)$ & $C(335)$ & $179.37(0.52)$ \\
\hline$C(332)$ & $C(333)$ & $C(334)$ & $\mathrm{F}(334)$ & $177.54(0.47)$ \\
\hline$C(332)$ & $C(333)$ & $C(334)$ & $C(335)$ & $-0.14(0.79)$ \\
\hline $\mathrm{F}(334)$ & $C(334)$ & $C(335)$ & $F(335)$ & $1.26(0.88)$ \\
\hline $\mathrm{F}(334)$ & $C(334)$ & $C(335)$ & $C(336)$ & $-177.52(0.53)$ \\
\hline$C(333)$ & $C(334)$ & $C(335)$ & $F(335)$ & $178.90(0.51)$ \\
\hline$C(333)$ & $C(334)$ & $C(335)$ & $C(336)$ & $0.12(0.91)$ \\
\hline $\mathrm{F}(335)$ & $C(335)$ & $C(336)$ & $F(336)$ & $3.28(0.84)$ \\
\hline $\mathrm{F}(335)$ & $C(335)$ & $C(336)$ & $C(331)$ & $-177.13(0.53)$ \\
\hline$C(334)$ & $C(335)$ & $C(336)$ & $F(336)$ & $-177.96(0.53)$ \\
\hline$C(334)$ & $C(335)$ & $C(336)$ & $C(331)$ & 1.63 ( 0.97) \\
\hline$C(346)$ & $C(341)$ & $C(342)$ & $\mathrm{F}(342)$ & $178.25(0.51)$ \\
\hline$C(346)$ & $C(341)$ & $C(342)$ & $C(343)$ & $-2.96(0.88)$ \\
\hline $\mathrm{B}(3)$ & $C(341)$ & $C(342)$ & $\mathrm{F}(342)$ & $7.50(0.81)$ \\
\hline B(3) & $C(341)$ & $C(342)$ & $C(343)$ & $-173.72(0.60)$ \\
\hline$C(342)$ & $C(341)$ & $C(346)$ & $\mathrm{F}(346)$ & $-178.61(0.52)$ \\
\hline$C(342)$ & $C(341)$ & $C(346)$ & $C(345)$ & $0.26(0.89)$ \\
\hline $\mathrm{B}(3)$ & $C(341)$ & $C(346)$ & $\mathrm{F}(346)$ & $-8.50(0.90)$ \\
\hline $\mathrm{B}(3)$ & $C(341)$ & $C(346)$ & $C(345)$ & $170.37(0.61)$ \\
\hline$C(342)$ & $\mathrm{C}(341)$ & $\mathrm{B}(3)$ & $C(311)$ & $-167.05(0.49)$ \\
\hline$C(342)$ & $C(341)$ & $\mathrm{B}(3)$ & $C(321)$ & $-52.88(0.75)$ \\
\hline$C(342)$ & $C(341)$ & $\mathrm{B}(3)$ & $C(331)$ & $70.51(0.61)$ \\
\hline$C(346)$ & $C(341)$ & $\mathrm{B}(3)$ & $C(311)$ & $23.63(0.80)$ \\
\hline$C(346)$ & $C(341)$ & $\mathrm{B}(3)$ & $C(321)$ & $137.80(0.60)$ \\
\hline$C(346)$ & $C(341)$ & $\mathrm{B}(3)$ & $C(331)$ & $-98.81(0.65)$ \\
\hline $\mathrm{F}(342)$ & $C(342)$ & $C(343)$ & $\mathrm{F}(343)$ & $0.49(0.93)$ \\
\hline$F(342)$ & $C(342)$ & $C(343)$ & $C(344)$ & $-177.44(0.60)$ \\
\hline$C(341)$ & $C(342)$ & $C(343)$ & $\mathrm{F}(343)$ & $-178.33(0.56)$ \\
\hline$C(341)$ & $C(342)$ & $C(343)$ & $C(344)$ & $3.75(1.06)$ \\
\hline$F(343)$ & $C(343)$ & $C(344)$ & $F(344)$ & $-1.13(1.02)$ \\
\hline $\mathrm{F}(343)$ & $C(343)$ & $C(344)$ & $C(345)$ & $-179.53(0.63)$ \\
\hline$C(342)$ & $C(343)$ & $C(344)$ & $\mathrm{F}(344)$ & $176.83(0.62)$ \\
\hline$C(342)$ & $C(343)$ & $C(344)$ & $C(345)$ & $-1.58(1.07)$ \\
\hline $\mathrm{F}(344)$ & $C(344)$ & $C(345)$ & $\mathrm{F}(345)$ & $-0.55(1.06)$ \\
\hline $\mathrm{F}(344)$ & $C(344)$ & $C(345)$ & $C(346)$ & $-179.36(0.62)$ \\
\hline$C(343)$ & $C(344)$ & $C(345)$ & $\mathrm{F}(345)$ & $177.83(0.62)$ \\
\hline$C(343)$ & $C(344)$ & $C(345)$ & $C(346)$ & $-0.98(1.08)$ \\
\hline $\mathrm{F}(345)$ & $C(345)$ & $C(346)$ & $F(346)$ & $1.78(0.90)$ \\
\hline $\mathrm{F}(345)$ & $C(345)$ & $C(346)$ & $C(341)$ & $-177.14(0.57)$ \\
\hline$C(344)$ & $C(345)$ & $C(346)$ & $\mathrm{F}(346)$ & $-179.42(0.62)$ \\
\hline$C(344)$ & $C(345)$ & $C(346)$ & $C(341)$ & 1.66 ( 1.08) \\
\hline C(416) & $\mathrm{C}(411)$ & $C(412)$ & $F(412)$ & $-176.36(0.47)$ \\
\hline$C(416)$ & $C(411)$ & $C(412)$ & $C(413)$ & $3.83(0.81)$ \\
\hline $\mathrm{B}(4)$ & $\mathrm{C}(411)$ & $C(412)$ & $F(412)$ & $-1.88(0.78)$ \\
\hline $\mathrm{B}(4)$ & $C(411)$ & $C(412)$ & $C(413)$ & $178.31(0.55)$ \\
\hline C(412) & $\mathrm{C}(411)$ & $C(416)$ & $F(416)$ & $175.97(0.46)$ \\
\hline$C(412)$ & $C(411)$ & $C(416)$ & $C(415)$ & $-4.02(0.79)$ \\
\hline $\mathrm{B}(4)$ & $\mathrm{C}(411)$ & $C(416)$ & $F(416)$ & $1.87(0.83)$ \\
\hline $\mathrm{B}(4)$ & $\mathrm{C}(411)$ & $C(416)$ & $C(415)$ & $-178.12(0.54)$ \\
\hline C(412) & $\mathrm{C}(411)$ & $\mathrm{B}(4)$ & $\mathrm{C}(421)$ & $-70.23(0.63)$ \\
\hline$C(412)$ & $\mathrm{C}(411)$ & $\mathrm{B}(4)$ & $C(431)$ & $51.76(0.71)$ \\
\hline$C(412)$ & $\mathrm{C}(411)$ & $\mathrm{B}(4)$ & $C(441)$ & $166.55(0.51)$ \\
\hline$C(416)$ & $C(411)$ & $\mathrm{B}(4)$ & $C(421)$ & $103.43(0.59)$ \\
\hline
\end{tabular}




\begin{tabular}{|c|c|c|c|c|}
\hline$C(416)$ & $C(411)$ & $\mathrm{B}(4)$ & $C(431)$ & $-134.58(0.55)$ \\
\hline$C(416)$ & $C(411)$ & $\mathrm{B}(4)$ & $C(441)$ & $-19.79(0.83)$ \\
\hline$F(412)$ & $C(412)$ & $C(413)$ & $F(413)$ & $-2.27(0.79)$ \\
\hline$F(412)$ & $C(412)$ & $C(413)$ & $C(414)$ & $178.84(0.49)$ \\
\hline$C(411)$ & $C(412)$ & $C(413)$ & $\mathrm{F}(413)$ & $177.54(0.52)$ \\
\hline$C(411)$ & $C(412)$ & $C(413)$ & $C(414)$ & $-1.35(0.90)$ \\
\hline$F(413)$ & $C(413)$ & $C(414)$ & $F(414)$ & $-0.72(0.82)$ \\
\hline$F(413)$ & $C(413)$ & $C(414)$ & $C(415)$ & $179.75(0.50)$ \\
\hline$C(412)$ & $C(413)$ & $C(414)$ & $F(414)$ & $178.19(0.50)$ \\
\hline$C(412)$ & $C(413)$ & $C(414)$ & $C(415)$ & $-1.35(0.83)$ \\
\hline$F(414)$ & $C(414)$ & $C(415)$ & $F(415)$ & $1.88(0.78)$ \\
\hline$F(414)$ & $C(414)$ & $C(415)$ & $C(416)$ & $-178.41(0.48)$ \\
\hline$C(413)$ & $C(414)$ & $C(415)$ & $\mathrm{F}(415)$ & $-178.58(0.49)$ \\
\hline$C(413)$ & $C(414)$ & $C(415)$ & $C(416)$ & $1.14(0.81)$ \\
\hline$F(415)$ & $C(415)$ & $C(416)$ & $F(416)$ & $1.47(0.74)$ \\
\hline$F(415)$ & $C(415)$ & $C(416)$ & $C(411)$ & $-178.54(0.49)$ \\
\hline$C(414)$ & $C(415)$ & $C(416)$ & $F(416)$ & $-178.24(0.47)$ \\
\hline$C(414)$ & $C(415)$ & $C(416)$ & $C(411)$ & $1.75(0.85)$ \\
\hline$C(426)$ & $C(421)$ & $C(422)$ & $F(422)$ & $-178.74(0.52)$ \\
\hline$C(426)$ & $C(421)$ & $C(422)$ & $C(423)$ & $1.75(0.89)$ \\
\hline B(4) & $C(421)$ & $C(422)$ & $\mathrm{F}(422)$ & $11.50(0.87)$ \\
\hline $\mathrm{B}(4)$ & $\mathrm{C}(421)$ & $C(422)$ & $C(423)$ & $-168.01(0.61)$ \\
\hline$C(422)$ & $C(421)$ & $C(426)$ & $F(426)$ & $-177.33(0.51)$ \\
\hline$C(422)$ & $C(421)$ & $C(426)$ & $C(425)$ & $1.65(0.89)$ \\
\hline B(4) & $C(421)$ & $C(426)$ & $F(426)$ & $-7.12(0.81)$ \\
\hline B(4) & $C(421)$ & $C(426)$ & $C(425)$ & $171.85(0.60)$ \\
\hline$C(422)$ & $C(421)$ & $\mathrm{B}(4)$ & $C(411)$ & $97.55(0.63)$ \\
\hline$C(422)$ & $C(421)$ & $\mathrm{B}(4)$ & $C(431)$ & $-24.83(0.77)$ \\
\hline$C(422)$ & $C(421)$ & $\mathrm{B}(4)$ & $\mathrm{C}(441)$ & $-138.65(0.58)$ \\
\hline$C(426)$ & $C(421)$ & $\mathrm{B}(4)$ & $C(411)$ & $-71.32(0.61)$ \\
\hline$C(426)$ & $C(421)$ & $\mathrm{B}(4)$ & $C(431)$ & $166.30(0.49)$ \\
\hline$C(426)$ & $C(421)$ & B(4) & $\mathrm{C}(441)$ & $52.49(0.76)$ \\
\hline$F(422)$ & $C(422)$ & $C(423)$ & $\mathrm{F}(423)$ & $-3.11(0.91)$ \\
\hline$F(422)$ & $C(422)$ & $C(423)$ & $C(424)$ & $177.45(0.62)$ \\
\hline$C(421)$ & $C(422)$ & $C(423)$ & $\mathrm{F}(423)$ & $176.43(0.57)$ \\
\hline$C(421)$ & $C(422)$ & $C(423)$ & $C(424)$ & $-3.02(1.07)$ \\
\hline$F(423)$ & $C(423)$ & $C(424)$ & $\mathrm{F}(424)$ & $0.21(1.06)$ \\
\hline$F(423)$ & $C(423)$ & $C(424)$ & $C(425)$ & $-178.64(0.63)$ \\
\hline$C(422)$ & $\mathrm{C}(423)$ & $C(424)$ & $\mathrm{F}(424)$ & $179.65(0.62)$ \\
\hline$C(422)$ & $C(423)$ & $C(424)$ & $C(425)$ & $0.81(1.09)$ \\
\hline$F(424)$ & $C(424)$ & $C(425)$ & $F(425)$ & $1.37(1.03)$ \\
\hline$F(424)$ & $C(424)$ & $C(425)$ & $C(426)$ & $-176.52(0.61)$ \\
\hline$C(423)$ & $\mathrm{C}(424)$ & $C(425)$ & $\mathrm{F}(425)$ & $-179.77(0.64)$ \\
\hline$C(423)$ & $C(424)$ & $C(425)$ & $C(426)$ & $2.34(1.07)$ \\
\hline$F(425)$ & $C(425)$ & $C(426)$ & $F(426)$ & $-2.60(0.92)$ \\
\hline$F(425)$ & $C(425)$ & $C(426)$ & $C(421)$ & $178.40(0.57)$ \\
\hline$C(424)$ & $C(425)$ & $C(426)$ & $F(426)$ & $175.27(0.59)$ \\
\hline$C(424)$ & $C(425)$ & $C(426)$ & $C(421)$ & $-3.74(1.05)$ \\
\hline$C(436)$ & $C(431)$ & $C(432)$ & $F(432)$ & $-178.93(0.53)$ \\
\hline$C(436)$ & $C(431)$ & $C(432)$ & $C(433)$ & $0.73(0.86)$ \\
\hline B(4) & $C(431)$ & $C(432)$ & $F(432)$ & $-6.87(0.88)$ \\
\hline $\mathrm{B}(4)$ & $C(431)$ & $C(432)$ & $\mathrm{C}(433)$ & $172.79(0.56)$ \\
\hline$C(432)$ & $\mathrm{C}(431)$ & $C(436)$ & $F(436)$ & $178.31(0.53)$ \\
\hline$C(432)$ & $\mathrm{C}(431)$ & $C(436)$ & $C(435)$ & $-0.80(0.88)$ \\
\hline $\mathrm{B}(4)$ & $C(431)$ & $C(436)$ & $F(436)$ & $5.63(0.80)$ \\
\hline B(4) & $C(431)$ & $C(436)$ & $C(435)$ & $-173.47(0.58)$ \\
\hline
\end{tabular}




\begin{tabular}{|c|c|c|c|c|}
\hline$C(432)$ & $\mathrm{C}(431)$ & $\mathrm{B}(4)$ & $C(411)$ & $18.66(0.77)$ \\
\hline$C(432)$ & $C(431)$ & $\mathrm{B}(4)$ & $C(421)$ & $133.93(0.57)$ \\
\hline$C(432)$ & $C(431)$ & $\mathrm{B}(4)$ & $C(441)$ & $-104.35(0.63)$ \\
\hline$C(436)$ & $C(431)$ & $\mathrm{B}(4)$ & $C(411)$ & $-169.72(0.49)$ \\
\hline$C(436)$ & $\mathrm{C}(431)$ & $\mathrm{B}(4)$ & $C(421)$ & $-54.46(0.65)$ \\
\hline$C(436)$ & $C(431)$ & $\mathrm{B}(4)$ & $C(441)$ & $67.26(0.67)$ \\
\hline$F(432)$ & $C(432)$ & $C(433)$ & $F(433)$ & $1.07(0.87)$ \\
\hline$F(432)$ & $C(432)$ & $C(433)$ & $C(434)$ & $178.97(0.57)$ \\
\hline $\mathrm{C}(431)$ & $C(432)$ & $C(433)$ & $F(433)$ & $-178.61(0.55)$ \\
\hline$C(431)$ & $C(432)$ & $C(433)$ & $C(434)$ & $-0.71(1.02)$ \\
\hline$F(433)$ & $C(433)$ & $C(434)$ & $F(434)$ & $-0.70(0.96)$ \\
\hline$F(433)$ & $C(433)$ & $C(434)$ & $C(435)$ & $178.56(0.57)$ \\
\hline$C(432)$ & $C(433)$ & $C(434)$ & $F(434)$ & $-178.61(0.59)$ \\
\hline$C(432)$ & $C(433)$ & $C(434)$ & $C(435)$ & $0.65(0.99)$ \\
\hline$F(434)$ & $C(434)$ & $C(435)$ & $F(435)$ & $0.26(0.97)$ \\
\hline$F(434)$ & $C(434)$ & $C(435)$ & $C(436)$ & $178.57(0.59)$ \\
\hline$C(433)$ & $C(434)$ & $C(435)$ & $F(435)$ & $-179.01(0.60)$ \\
\hline$C(433)$ & $C(434)$ & $C(435)$ & $C(436)$ & $-0.70(0.97)$ \\
\hline$F(435)$ & $C(435)$ & $C(436)$ & $F(436)$ & $0.00(0.91)$ \\
\hline$F(435)$ & $C(435)$ & $C(436)$ & $\mathrm{C}(431)$ & $179.13(0.56)$ \\
\hline$C(434)$ & $C(435)$ & $C(436)$ & $F(436)$ & $-178.31(0.56)$ \\
\hline$C(434)$ & $C(435)$ & $C(436)$ & $C(431)$ & $0.82(1.01)$ \\
\hline$C(446)$ & $\mathrm{C}(441)$ & $C(442)$ & $F(442)$ & $178.52(0.56)$ \\
\hline$C(446)$ & $C(441)$ & $C(442)$ & $C(443)$ & $-0.36(0.93)$ \\
\hline $\mathrm{B}(4)$ & $C(441)$ & $C(442)$ & $F(442)$ & $1.06(0.83)$ \\
\hline B(4) & $C(441)$ & $C(442)$ & $C(443)$ & $-177.82(0.60)$ \\
\hline $\mathrm{C}(442)$ & $\mathrm{C}(441)$ & $C(446)$ & $\mathrm{F}(446)$ & $-179.47(0.57)$ \\
\hline$C(442)$ & $C(441)$ & $C(446)$ & $C(445)$ & $-0.23(0.99)$ \\
\hline $\mathrm{B}(4)$ & $C(441)$ & $C(446)$ & $\mathrm{F}(446)$ & $-2.27(1.05)$ \\
\hline B(4) & $C(441)$ & $C(446)$ & $C(445)$ & $176.97(0.64)$ \\
\hline$C(442)$ & $C(441)$ & $\mathrm{B}(4)$ & $C(411)$ & $-49.31(0.74)$ \\
\hline$C(442)$ & $C(441)$ & $\mathrm{B}(4)$ & $C(421)$ & $-165.65(0.53)$ \\
\hline$C(442)$ & $C(441)$ & B(4) & $C(431)$ & $73.08(0.67)$ \\
\hline$C(446)$ & $\mathrm{C}(441)$ & B(4) & $\mathrm{C}(411)$ & $133.57(0.68)$ \\
\hline$C(446)$ & $\mathrm{C}(441)$ & B(4) & $\mathrm{C}(421)$ & $17.23(0.91)$ \\
\hline$C(446)$ & $C(441)$ & B(4) & $\mathrm{C}(431)$ & $-104.04(0.70)$ \\
\hline$F(442)$ & $C(442)$ & $C(443)$ & $\mathrm{F}(443)$ & $1.09(0.93)$ \\
\hline$F(442)$ & $C(442)$ & $C(443)$ & $C(444)$ & $-178.73(0.62)$ \\
\hline $\mathrm{C}(441)$ & $C(442)$ & $C(443)$ & $\mathrm{F}(443)$ & $-179.99(0.47)$ \\
\hline$C(441)$ & $C(442)$ & $C(443)$ & $C(444)$ & $0.20(1.04)$ \\
\hline$F(443)$ & $C(443)$ & $C(444)$ & $F(444)$ & $-0.03(1.12)$ \\
\hline$F(443)$ & $C(443)$ & $C(444)$ & $\mathrm{C}(445)$ & $-179.26(0.65)$ \\
\hline $\mathrm{C}(442)$ & $C(443)$ & $C(444)$ & $\mathrm{F}(444)$ & $179.79(0.63)$ \\
\hline $\mathrm{C}(442)$ & $C(443)$ & $C(444)$ & $C(445)$ & $0.57(1.11)$ \\
\hline $\mathrm{F}(444)$ & $C(444)$ & $C(445)$ & $F(445)$ & $1.38(1.12)$ \\
\hline$F(444)$ & $C(444)$ & $C(445)$ & $C(446)$ & $179.64(0.66)$ \\
\hline$C(443)$ & $C(444)$ & $C(445)$ & $F(445)$ & $-179.40(0.67)$ \\
\hline$C(443)$ & $C(444)$ & $C(445)$ & $C(446)$ & $-1.14(1.14)$ \\
\hline$F(445)$ & $C(445)$ & $C(446)$ & $F(446)$ & $-1.42(0.98)$ \\
\hline$F(445)$ & $C(445)$ & $C(446)$ & $\mathrm{C}(441)$ & $179.29(0.65)$ \\
\hline $\mathrm{C}(444)$ & $C(445)$ & $C(446)$ & $\mathrm{F}(446)$ & $-179.72(0.65)$ \\
\hline$C(444)$ & $C(445)$ & $C(446)$ & $C(441)$ & $1.00(1.16)$ \\
\hline
\end{tabular}


Table S8. Positional Parameters and Their Estimated Standard Deviations for $\left[\mathrm{Re}(\mathrm{NMes})\left(\mathrm{PPh}_{3}\right)\right.$ saldach $\left.^{+}\right], \mathbf{7 a}$

\begin{tabular}{|c|c|c|c|c|}
\hline Atom & $\underline{\mathrm{x}}$ & $\mathrm{y}$ & $\underline{\mathrm{Z}}$ & $\left.\underline{\mathrm{U}\left(\AA^{2}\right.}{ }^{2}\right)$ \\
\hline $\operatorname{Re}(1)$ & $0 . \overline{1} 06305(12)$ & $0.270581(12)$ & $-0.014942(9)$ & $0.02615(9)$ \\
\hline $\operatorname{Re}(2)$ & $-0.399355(13)$ & $0.260102(12)$ & $-0.506641(9)$ & $0.02789(10)$ \\
\hline $\mathrm{Cl}(91)$ & $0.02600(18)$ & $0.06936(17)$ & $0.1835(2)$ & $0.129(2)$ \\
\hline $\mathrm{Cl}(92)$ & $-0.0128(3)$ & $0.0382(3)$ & $0.0778(2)$ & $0.171(3)$ \\
\hline $\mathrm{Cl}(93)$ & $0.2551(4)$ & $0.3625(4)$ & $0.3981(3)$ & $0.203(4)$ \\
\hline $\mathrm{Cl}(94)$ & $0.3324(6)$ & $0.4080(5)$ & $0.2800(4)$ & $0.236(7)$ \\
\hline $\mathrm{Cl}(95)$ & $0.52219(17)$ & $0.06489(15)$ & $0.70513(18)$ & $0.1037(15)$ \\
\hline $\mathrm{Cl}(96)$ & $0.4793(2)$ & $0.0273(2)$ & $0.60462(19)$ & $0.132(2)$ \\
\hline $\mathrm{Cl}(97)$ & $0.2462(4)$ & $0.6385(4)$ & $0.1119(3)$ & $0.164(4)$ \\
\hline $\mathrm{Cl}(98)$ & $0.1532(5)$ & $0.5941(4)$ & $0.2233(3)$ & $0.181(5)$ \\
\hline $\mathrm{P}(130)$ & $0.23490(8)$ & $0.28809(8)$ & $-0.02085(6)$ & $0.0233(5)$ \\
\hline $\mathrm{P}(230)$ & $-0.27092(8)$ & $0.28141(8)$ & $-0.52064(6)$ & $0.0246(5)$ \\
\hline$F(312)$ & $0.2502(2)$ & $0.2920(2)$ & $0.55995(17)$ & $0.0495(14)$ \\
\hline $\mathrm{F}(313)$ & $0.2262(2)$ & $0.4424(2)$ & $0.5323(2)$ & $0.073(2)$ \\
\hline$F(314)$ & $0.0740(3)$ & $0.5505(2)$ & $0.5461(2)$ & $0.0739(18)$ \\
\hline$F(315)$ & $-0.0545(2)$ & $0.5022(2)$ & $0.59074(19)$ & $0.0615(16)$ \\
\hline$F(316)$ & $-0.03277(19)$ & $0.35406(19)$ & $0.62091(16)$ & $0.0443(14)$ \\
\hline $\mathrm{F}(322)$ & $0.2972(2)$ & $0.1497(2)$ & $0.52540(17)$ & $0.0547(17)$ \\
\hline$F(323)$ & $0.3213(3)$ & $0.1506(3)$ & $0.40300(19)$ & $0.086(2)$ \\
\hline$F(324)$ & $0.1936(3)$ & $0.2130(3)$ & $0.33329(15)$ & $0.088(3)$ \\
\hline$F(325)$ & $0.0386(3)$ & $0.2737(2)$ & $0.39205(15)$ & $0.067(2)$ \\
\hline$F(326)$ & $0.0132(2)$ & $0.2776(2)$ & $0.51448(13)$ & $0.0410(13)$ \\
\hline$F(332)$ & $0.05850(18)$ & $0.11730(18)$ & $0.57318(13)$ & $0.0340(13)$ \\
\hline$F(333)$ & $-0.0604(2)$ & $0.0743(2)$ & $0.64426(14)$ & $0.0420(14)$ \\
\hline$F(334)$ & $-0.1324(2)$ & $0.1197(2)$ & $0.75554(15)$ & $0.0510(16)$ \\
\hline $\mathrm{F}(335)$ & $-0.0779(2)$ & $0.2082(2)$ & $0.79506(15)$ & $0.0578(18)$ \\
\hline$F(336)$ & $0.0435(2)$ & $0.2509(2)$ & $0.72564(14)$ & $0.0442(14)$ \\
\hline$F(342)$ & $0.22105(19)$ & $0.05362(18)$ & $0.61062(15)$ & $0.0406(14)$ \\
\hline$F(343)$ & $0.3301(2)$ & $-0.0449(2)$ & $0.68870(18)$ & $0.0542(16)$ \\
\hline$F(344)$ & $0.3694(2)$ & $0.0045(2)$ & $0.77497(17)$ & $0.0545(14)$ \\
\hline$F(345)$ & $0.2998(2)$ & $0.1575(2)$ & $0.77810(15)$ & $0.0473(14)$ \\
\hline$F(346)$ & $0.1985(2)$ & $0.25818(18)$ & $0.69687(15)$ & $0.0425(14)$ \\
\hline$F(412)$ & $0.4432(2)$ & $-0.2518(2)$ & $0.77796(14)$ & $0.0467(16)$ \\
\hline $\mathrm{F}(413)$ & $0.5610(3)$ & $-0.2079(2)$ & $0.70292(14)$ & $0.0630(19)$ \\
\hline$F(414)$ & $0.6232(2)$ & $-0.1227(2)$ & $0.73891(15)$ & $0.0524(14)$ \\
\hline $\mathrm{F}(415)$ & $0.5600(2)$ & $-0.0796(2)$ & $0.85288(14)$ & $0.0431(14)$ \\
\hline$F(416)$ & $0.44348(18)$ & $-0.12263(18)$ & $0.92842(12)$ & $0.0324(13)$ \\
\hline$F(422)$ & $0.2884(2)$ & $-0.25957(18)$ & $0.81429(15)$ & $0.0438(14)$ \\
\hline$F(423)$ & $0.1868(2)$ & $-0.1570(2)$ & $0.73437(16)$ & $0.0529(16)$ \\
\hline$F(424)$ & $0.1185(2)$ & $-0.0041(2)$ & $0.73930(17)$ & $0.0584(16)$ \\
\hline$F(425)$ & $0.1610(2)$ & $0.04204(19)$ & $0.82641(17)$ & $0.0516(16)$ \\
\hline$F(426)$ & $0.27498(19)$ & $-0.05799(17)$ & $0.89975(14)$ & $0.0382(13)$ \\
\hline$F(432)$ & $0.52735(19)$ & $-0.35663(19)$ & $0.87826(15)$ & $0.0422(14)$ \\
\hline$F(433)$ & $0.5492(2)$ & $-0.5053(2)$ & $0.90865(18)$ & $0.0584(16)$ \\
\hline $\mathrm{F}(434)$ & $0.4226(3)$ & $-0.5540(2)$ & $0.9603(2)$ & $0.0670(18)$ \\
\hline$F(435)$ & $0.2705(2)$ & $-0.4461(2)$ & $0.9814(2)$ & $0.072(2)$ \\
\hline$F(436)$ & $0.2467(2)$ & $-0.2965(2)$ & $0.95255(17)$ & $0.0494(14)$ \\
\hline$F(442)$ & $0.49049(18)$ & $-0.28339(18)$ & $0.98527(13)$ & $0.0353(13)$ \\
\hline $\mathrm{F}(443)$ & $0.4741(2)$ & $-0.2838(2)$ & $1.10831(14)$ & $0.0559(18)$ \\
\hline$F(444)$ & $0.3247(3)$ & $-0.2221(2)$ & $1.17269(14)$ & $0.076(2)$ \\
\hline
\end{tabular}




\begin{tabular}{|c|c|c|c|c|}
\hline Atom & $\underline{x}$ & $\mathrm{y}$ & $\underline{\mathrm{z}}$ & \left.${\underline{\mathrm{U}}\left(\AA^{2}\right.}^{2}\right)$ \\
\hline$F(446)$ & $0.2049(2)$ & $-0.1556(2)$ & $0.98755(16)$ & $0.0520(17)$ \\
\hline $\mathrm{O}(11)$ & $0.0651(2)$ & $0.3276(2)$ & $0.05658(15)$ & $0.0269(14)$ \\
\hline $\mathrm{O}(21)$ & $-0.4370(2)$ & $0.3207(2)$ & $-0.43776(15)$ & $0.0259(13)$ \\
\hline $\mathrm{O}(119)$ & $0.1445(2)$ & $0.1810(2)$ & $0.05801(16)$ & $0.0291(14)$ \\
\hline $\mathrm{O}(219)$ & $-0.3565(2)$ & $0.1727(2)$ & $-0.43204(16)$ & $0.0320(14)$ \\
\hline $\mathrm{N}(18)$ & $-0.0050(3)$ & $0.2517(3)$ & $0.0029(2)$ & $0.034(2)$ \\
\hline $\mathrm{N}(28)$ & $-0.5124(3)$ & $0.2406(3)$ & $-0.4820(3)$ & $0.041(2)$ \\
\hline $\mathrm{N}(112)$ & $0.1518(3)$ & $0.1828(3)$ & $-0.0651(2)$ & $0.036(2)$ \\
\hline $\mathrm{N}(120)$ & $0.0732(3)$ & $0.3485(3)$ & $-0.08041(19)$ & $0.0288(18)$ \\
\hline $\mathrm{N}(212)$ & $-0.3573(3)$ & $0.1689(3)$ & $-0.5522(2)$ & $0.044(2)$ \\
\hline $\mathrm{N}(220)$ & $-0.4355(3)$ & $0.3345(3)$ & $-0.57102(19)$ & $0.0325(18)$ \\
\hline C(11) & $0.0150(3)$ & $0.3111(3)$ & $0.1079(2)$ & $0.029(2)$ \\
\hline$C(12)$ & $0.0181(3)$ & $0.3291(3)$ & $0.1639(2)$ & $0.036(2)$ \\
\hline$C(13)$ & $-0.0334(4)$ & $0.3148(4)$ & $0.2199(3)$ & $0.045(3)$ \\
\hline$C(14)$ & $-0.0909(4)$ & $0.2840(4)$ & $0.2212(3)$ & $0.051(3)$ \\
\hline$C(15)$ & $-0.0948(4)$ & $0.2661(4)$ & $0.1663(3)$ & $0.047(3)$ \\
\hline$C(16)$ & $-0.0423(3)$ & $0.2779(3)$ & $0.1091(3)$ & $0.036(2)$ \\
\hline$C(17)$ & $-0.0511(4)$ & $0.2562(3)$ & $0.0556(3)$ & $0.038(2)$ \\
\hline$C(19)$ & $-0.0370(4)$ & $0.2432(4)$ & $-0.0487(3)$ & $0.051(3)$ \\
\hline$C(21)$ & $-0.4865(3)$ & $0.3068(3)$ & $-0.3841(2)$ & $0.027(2)$ \\
\hline $\mathrm{C}(22)$ & $-0.4800(3)$ & $0.3271(3)$ & $-0.3314(2)$ & $0.034(2)$ \\
\hline$C(23)$ & $-0.5307(4)$ & $0.3175(4)$ & $-0.2745(3)$ & $0.049(3)$ \\
\hline$C(24)$ & $-0.5896(4)$ & $0.2876(4)$ & $-0.2694(3)$ & $0.057(3)$ \\
\hline$C(25)$ & $-0.5974(4)$ & $0.2669(4)$ & $-0.3215(3)$ & $0.054(3)$ \\
\hline$C(26)$ & $-0.5449(3)$ & $0.2733(3)$ & $-0.3788(3)$ & $0.038(2)$ \\
\hline$C(27)$ & $-0.5576(3)$ & $0.2497(3)$ & $-0.4295(3)$ & $0.041(2)$ \\
\hline C(29) & $-0.5443(4)$ & $0.2276(4)$ & $-0.5312(3)$ & $0.058(3)$ \\
\hline$C(110)$ & $0.0277(4)$ & $0.1817(4)$ & $-0.0866(3)$ & $0.054(3)$ \\
\hline$C(111)$ & $0.1039(4)$ & $0.1974(4)$ & $-0.1175(3)$ & $0.045(3)$ \\
\hline C(113) & $0.2023(4)$ & $0.1119(3)$ & $-0.0481(3)$ & $0.040(3)$ \\
\hline C(114) & $0.2382(3)$ & $0.0774(3)$ & $0.0098(3)$ & $0.033(2)$ \\
\hline$C(115)$ & $0.3009(4)$ & $0.0035(3)$ & $0.0168(3)$ & $0.044(3)$ \\
\hline$C(116)$ & $0.3294(4)$ & $-0.0346(4)$ & $0.0737(4)$ & $0.053(3)$ \\
\hline$C(117)$ & $0.2954(4)$ & $-0.0016(4)$ & $0.1270(3)$ & $0.053(3)$ \\
\hline C(118) & $0.2335(4)$ & $0.0703(4)$ & $0.1214(3)$ & $0.047(3)$ \\
\hline C(119) & $0.2040(3)$ & $0.1118(3)$ & $0.0626(3)$ & $0.030(2)$ \\
\hline$C(121)$ & $0.0392(3)$ & $0.4068(3)$ & $-0.1296(2)$ & $0.030(2)$ \\
\hline C(122) & $0.0811(4)$ & $0.4129(4)$ & $-0.1929(3)$ & $0.041(3)$ \\
\hline$C(123)$ & $0.0406(4)$ & $0.4707(4)$ & $-0.2412(3)$ & $0.045(3)$ \\
\hline$C(124)$ & $-0.0367(4)$ & $0.5222(4)$ & $-0.2300(3)$ & $0.045(3)$ \\
\hline$C(125)$ & $-0.0755(4)$ & $0.5181(3)$ & $-0.1671(3)$ & $0.040(3)$ \\
\hline$C(126)$ & $-0.0401(3)$ & $0.4621(3)$ & $-0.1160(3)$ & $0.034(2)$ \\
\hline$C(127)$ & $0.1676(4)$ & $0.3608(4)$ & $-0.2066(3)$ & $0.053(3)$ \\
\hline $\mathrm{C}(128)$ & $-0.0804(5)$ & $0.5849(4)$ & $-0.2837(3)$ & $0.066(3)$ \\
\hline C(129) & $-0.0822(4)$ & $0.4584(4)$ & $-0.0483(3)$ & $0.047(3)$ \\
\hline C(131) & $0.3259(3)$ & $0.2097(3)$ & $-0.0422(2)$ & $0.026(2)$ \\
\hline$C(132)$ & $0.3869(3)$ & $0.1665(3)$ & $-0.0026(2)$ & $0.031(2)$ \\
\hline$C(133)$ & $0.4543(4)$ & $0.1056(3)$ & $-0.0204(3)$ & $0.041(2)$ \\
\hline C(134) & $0.4621(4)$ & $0.0880(4)$ & $-0.0776(3)$ & $0.042(3)$ \\
\hline C(135) & $0.4024(3)$ & $0.1301(3)$ & $-0.1177(3)$ & $0.037(2)$ \\
\hline
\end{tabular}




\begin{tabular}{|c|c|c|c|c|}
\hline$\underline{\text { Atom }}$ & $\underline{x}$ & y & $\underline{\mathrm{Z}}$ & $\left.\underline{\mathrm{U}\left(\AA^{2}\right.}{ }^{2}\right)$ \\
\hline C(137) & $0.2428(3)$ & $0.3748(3)$ & $-0.0791(2)$ & $0.032(2)$ \\
\hline C(138) & $0.3154(4)$ & $0.3755(4)$ & $-0.1187(3)$ & $0.050(3)$ \\
\hline C(139) & $0.3184(4)$ & $0.4449(4)$ & $-0.1603(3)$ & $0.062(3)$ \\
\hline$C(140)$ & $0.2525(5)$ & $0.5118(4)$ & $-0.1614(3)$ & $0.067(3)$ \\
\hline C(141) & $0.1796(4)$ & $0.5125(3)$ & $-0.1221(3)$ & $0.049(3)$ \\
\hline$C(142)$ & $0.1755(4)$ & $0.4442(3)$ & $-0.0810(2)$ & $0.038(2)$ \\
\hline C(143) & $0.2518(3)$ & $0.3010(3)$ & $0.0530(2)$ & $0.024(2)$ \\
\hline C(144) & $0.2924(4)$ & $0.3487(3)$ & $0.0498(3)$ & $0.038(3)$ \\
\hline$C(145)$ & $0.3089(4)$ & $0.3548(4)$ & $0.1059(3)$ & $0.048(3)$ \\
\hline$C(146)$ & $0.2852(4)$ & $0.3138(4)$ & $0.1640(3)$ & $0.049(3)$ \\
\hline C(147) & $0.2450(4)$ & $0.2670(4)$ & $0.1671(3)$ & $0.043(3)$ \\
\hline$C(148)$ & $0.2264(3)$ & $0.2605(3)$ & $0.1124(2)$ & $0.032(2)$ \\
\hline$C(210)$ & $-0.4842(5)$ & $0.1630(5)$ & $-0.5648(4)$ & $0.075(4)$ \\
\hline $\mathrm{C}(211)$ & $-0.4081(4)$ & $0.1777(5)$ & $-0.6001(3)$ & $0.066(4)$ \\
\hline$C(213)$ & $-0.3039(4)$ & $0.0995(4)$ & $-0.5356(3)$ & $0.049(3)$ \\
\hline$C(214)$ & $-0.2636(4)$ & $0.0676(3)$ & $-0.4796(3)$ & $0.040(3)$ \\
\hline$C(215)$ & $-0.1990(4)$ & $-0.0045(4)$ & $-0.4718(4)$ & $0.056(3)$ \\
\hline$C(216)$ & $-0.1667(4)$ & $-0.0388(4)$ & $-0.4164(4)$ & $0.061(3)$ \\
\hline C(217) & $-0.1987(4)$ & $-0.0030(4)$ & $-0.3645(3)$ & $0.056(3)$ \\
\hline C(218) & $-0.2623(4)$ & $0.0674(4)$ & $-0.3702(3)$ & $0.045(3)$ \\
\hline C(219) & $-0.2944(3)$ & $0.1044(3)$ & $-0.4282(3)$ & $0.033(2)$ \\
\hline$C(221)$ & $-0.4694(3)$ & $0.3934(3)$ & $-0.6211(2)$ & $0.031(2)$ \\
\hline$C(222)$ & $-0.5443(3)$ & $0.4530(3)$ & $-0.6075(3)$ & $0.034(2)$ \\
\hline$C(223)$ & $-0.5789(4)$ & $0.5104(3)$ & $-0.6572(3)$ & $0.036(2)$ \\
\hline$C(224)$ & $-0.5435(4)$ & $0.5136(4)$ & $-0.7205(3)$ & $0.046(3)$ \\
\hline$C(225)$ & $-0.4697(4)$ & $0.4573(4)$ & $-0.7336(3)$ & $0.046(3)$ \\
\hline$C(226)$ & $-0.4308(4)$ & $0.3960(4)$ & $-0.6848(3)$ & $0.045(3)$ \\
\hline C(227) & $-0.5834(4)$ & $0.4505(4)$ & $-0.5397(3)$ & $0.048(3)$ \\
\hline $\mathrm{C}(228)$ & $-0.5844(5)$ & $0.5789(4)$ & $-0.7752(3)$ & $0.065(3)$ \\
\hline C(229) & $-0.3490(4)$ & $0.3376(5)$ & $-0.7006(3)$ & $0.083(4)$ \\
\hline $\mathrm{C}(231)$ & $-0.2695(3)$ & $0.3665(3)$ & $-0.5823(2)$ & $0.033(2)$ \\
\hline$C(232)$ & $-0.3365(4)$ & $0.4355(3)$ & $-0.5812(2)$ & $0.035(2)$ \\
\hline$C(233)$ & $-0.3368(5)$ & $0.5037(4)$ & $-0.6240(3)$ & $0.057(3)$ \\
\hline$C(234)$ & $-0.2709(6)$ & $0.5047(4)$ & $-0.6711(3)$ & $0.081(4)$ \\
\hline$C(235)$ & $-0.2028(5)$ & $0.4376(4)$ & $-0.6724(3)$ & $0.088(4)$ \\
\hline$C(236)$ & $-0.2009(4)$ & $0.3681(4)$ & $-0.6280(3)$ & $0.063(3)$ \\
\hline$C(237)$ & $-0.1783(3)$ & $0.2034(3)$ & $-0.5421(2)$ & $0.0246(18$ \\
\hline C(238) & $-0.1160(3)$ & $0.1667(3)$ & $-0.5049(3)$ & $0.034(2)$ \\
\hline C(239) & $-0.0476(4)$ & $0.1067(4)$ & $-0.5229(3)$ & $0.044(3)$ \\
\hline$C(240)$ & $-0.0410(4)$ & $0.0835(4)$ & $-0.5770(3)$ & $0.047(3)$ \\
\hline$C(241)$ & $-0.1038(4)$ & $0.1193(4)$ & $-0.6145(3)$ & $0.044(3)$ \\
\hline$C(242)$ & $-0.1717(3)$ & $0.1786(3)$ & $-0.5969(2)$ & $0.037(2)$ \\
\hline$C(243)$ & $-0.2507(3)$ & $0.3006(3)$ & $-0.4518(2)$ & $0.026(2)$ \\
\hline$C(244)$ & $-0.2103(3)$ & $0.3506(3)$ & $-0.4604(3)$ & $0.036(2)$ \\
\hline$C(245)$ & $-0.1912(4)$ & $0.3635(4)$ & $-0.4086(3)$ & $0.051(3)$ \\
\hline$C(246)$ & $-0.2108(4)$ & $0.3251(4)$ & $-0.3482(3)$ & $0.048(3)$ \\
\hline C(247) & $-0.2513(4)$ & $0.2739(4)$ & $-0.3382(3)$ & $0.041(3)$ \\
\hline $\mathrm{C}(248)$ & $-0.2717(3)$ & $0.2619(3)$ & $-0.3898(2)$ & $0.029(2)$ \\
\hline$C(311)$ & $0.1112(3)$ & $0.3130(3)$ & $0.5934(2)$ & $0.029(2)$ \\
\hline$C(312)$ & $0.1732(4)$ & $0.3428(3)$ & $0.5689(3)$ & $0.037(2)$ \\
\hline
\end{tabular}




\begin{tabular}{|c|c|c|c|c|}
\hline Atom & $\underline{x}$ & $\mathrm{y}$ & $\underline{\mathrm{Z}}$ & $\left.\underline{\mathrm{U}\left(\AA^{2}\right.}\right)$ \\
\hline$C(314)$ & $0.0861(4)$ & $0.4740(4)$ & $0.5615(3)$ & $0.048(3)$ \\
\hline$C(315)$ & $0.0215(4)$ & $0.4497(4)$ & $0.5844(3)$ & $0.043(3)$ \\
\hline$C(316)$ & $0.0353(3)$ & $0.3717(3)$ & $0.5989(3)$ & $0.033(2)$ \\
\hline$C(321)$ & $0.1545(4)$ & $0.2109(3)$ & $0.5287(2)$ & $0.031(2)$ \\
\hline$C(322)$ & $0.2299(4)$ & $0.1815(4)$ & $0.4948(3)$ & $0.044(3)$ \\
\hline$C(323)$ & $0.2436(5)$ & $0.1834(4)$ & $0.4307(3)$ & $0.056(3)$ \\
\hline C(324) & $0.1804(5)$ & $0.2123(4)$ & $0.3962(3)$ & $0.059(4)$ \\
\hline C(325) & $0.1026(4)$ & $0.2443(4)$ & $0.4251(3)$ & $0.046(3)$ \\
\hline$C(326)$ & $0.0908(4)$ & $0.2442(3)$ & $0.4892(2)$ & $0.033(2)$ \\
\hline$C(331)$ & $0.0558(3)$ & $0.1912(3)$ & $0.6433(2)$ & $0.025(2)$ \\
\hline C(332) & $0.0266(3)$ & $0.1437(3)$ & $0.6274(2)$ & $0.027(2)$ \\
\hline C(333) & $-0.0350(3)$ & $0.1190(3)$ & $0.6635(2)$ & $0.029(2)$ \\
\hline$C(334)$ & $-0.0703(3)$ & $0.1407(3)$ & $0.7208(2)$ & $0.034(2)$ \\
\hline C(335) & $-0.0428(4)$ & $0.1855(4)$ & $0.7395(2)$ & $0.037(2)$ \\
\hline C(336) & $0.0184(3)$ & $0.2084(3)$ & $0.7020(2)$ & $0.033(2)$ \\
\hline$C(341)$ & $0.2043(3)$ & $0.1631(3)$ & $0.6473(2)$ & $0.028(2)$ \\
\hline$C(342)$ & $0.2407(3)$ & $0.0827(3)$ & $0.6502(3)$ & $0.031(2)$ \\
\hline$C(343)$ & $0.2963(4)$ & $0.0314(3)$ & $0.6899(3)$ & $0.038(3)$ \\
\hline C(344) & $0.3173(4)$ & $0.0559(4)$ & $0.7336(3)$ & $0.041(3)$ \\
\hline$C(345)$ & $0.2826(4)$ & $0.1318(3)$ & $0.7343(3)$ & $0.035(2)$ \\
\hline$C(346)$ & $0.2287(3)$ & $0.1832(3)$ & $0.6917(3)$ & $0.031(2)$ \\
\hline C(411) & $0.4397(3)$ & $-0.1948(3)$ & $0.8594(2)$ & $0.026(2)$ \\
\hline C(412) & $0.4716(3)$ & $-0.2106(3)$ & $0.7996(2)$ & $0.032(2)$ \\
\hline C(413) & $0.5314(4)$ & $-0.1876(3)$ & $0.7593(2)$ & $0.038(3)$ \\
\hline C(414) & $0.5626(3)$ & $-0.1444(3)$ & $0.7770(2)$ & $0.038(2)$ \\
\hline C(415) & $0.5315(3)$ & $-0.1238(3)$ & $0.8347(2)$ & $0.031(2)$ \\
\hline C(416) & $0.4718(3)$ & $-0.1475(3)$ & $0.8735(2)$ & $0.025(2)$ \\
\hline$C(421)$ & $0.2881(3)$ & $-0.1654(3)$ & $0.8626(2)$ & $0.028(2)$ \\
\hline$C(422)$ & $0.2608(3)$ & $-0.1850(3)$ & $0.8188(3)$ & $0.034(2)$ \\
\hline C(423) & $0.2060(4)$ & $-0.1326(4)$ & $0.7774(3)$ & $0.038(3)$ \\
\hline$C(424)$ & $0.1721(4)$ & $-0.0566(4)$ & $0.7797(3)$ & $0.042(3)$ \\
\hline$C(425)$ & $0.1942(4)$ & $-0.0332(3)$ & $0.8232(3)$ & $0.037(3)$ \\
\hline$C(426)$ & $0.2519(3)$ & $-0.0865(3)$ & $0.8614(3)$ & $0.031(2)$ \\
\hline C(431) & $0.3851(3)$ & $-0.3174(3)$ & $0.9124(2)$ & $0.031(2)$ \\
\hline C(432) & $0.4596(4)$ & $-0.3739(3)$ & $0.9031(3)$ & $0.035(3)$ \\
\hline C(433) & $0.4729(4)$ & $-0.4529(4)$ & $0.9182(3)$ & $0.043(3)$ \\
\hline C(434) & $0.4104(4)$ & $-0.4780(3)$ & $0.9443(3)$ & $0.045(3)$ \\
\hline C(435) & $0.3334(4)$ & $-0.4239(4)$ & $0.9550(3)$ & $0.049(3)$ \\
\hline$C(436)$ & $0.3231(4)$ & $-0.3465(3)$ & $0.9394(3)$ & $0.040(2)$ \\
\hline C(441) & $0.3489(4)$ & $-0.2184(3)$ & $0.9794(2)$ & $0.031(2)$ \\
\hline C(442) & $0.4151(4)$ & $-0.2505(3)$ & $1.0134(2)$ & $0.031(2)$ \\
\hline C(443) & $0.4081(4)$ & $-0.2520(4)$ & $1.0770(3)$ & $0.040(3)$ \\
\hline C(444) & $0.3325(5)$ & $-0.2208(4)$ & $1.1101(3)$ & $0.053(3)$ \\
\hline$C(445)$ & $0.2667(4)$ & $-0.1899(4)$ & $1.0786(3)$ & $0.050(3)$ \\
\hline C(446) & $0.2753(4)$ & $-0.1884(4)$ & $1.0140(3)$ & $0.040(2)$ \\
\hline C(912) & $0.0556(7)$ & $0.0211(8)$ & $0.1248(5)$ & $0.134(8)$ \\
\hline C(934) & $0.2656(10)$ & $0.4330(9)$ & $0.3392(6)$ & $0.120(7)$ \\
\hline C(956) & $0.5517(6)$ & $0.0369(6)$ & $0.6354(4)$ & $0.100(6)$ \\
\hline C(978) & $0.2208(10)$ & $0.5729(10)$ & $0.1639(8)$ & $0.096(6)$ \\
\hline $\mathrm{B}(3)$ & $0.1307(4)$ & $0.2193(4)$ & $0.6030(3)$ & $0.028(2)$ \\
\hline
\end{tabular}




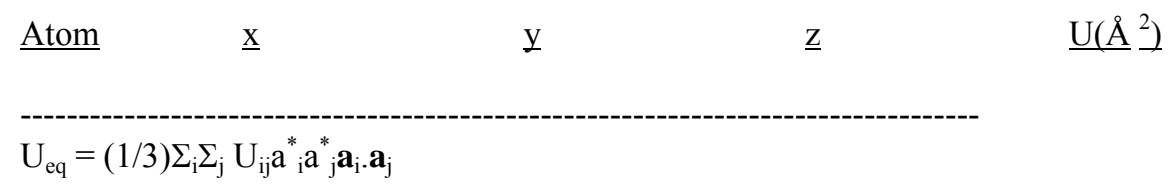

